\title{
S1 Calculation
}

Medicare Current Beneficiary Survey (MCBS) Descriptive Analysis: This study analyzed the 2015 MCBS Public Use File (n=12,311), a nationally representative, publicly accessible database from the Centers for Medicare \& Medicaid Services. The authors conducted a descriptive analysis that accounted for the complex sample design with survey weights. The study found that $80 \%$ of Medicare beneficiaries indicated that they lived in a metro area, and $73 \%$ were still driving themselves to the doctor's office. 
S1 Table. Estimated Number, Prevalence, and Density (MDPP-Eligible per Square Mile of Land

Area) of MDPP-Eligible Part B Beneficiaries, by State

\begin{tabular}{|c|c|c|c|c|}
\hline State & Estimated MDPP eligible & Prevalence & Land area (square miles) & $\begin{array}{l}\text { Density (eligible } \\
\text { beneficiaries per square } \\
\text { mile of land area) }\end{array}$ \\
\hline 50 states $+\mathrm{DC}+\mathrm{PR}+\mathrm{VI}$ & $5,271,473$ & $28.5 \%$ & $3,535,463.53$ & 1.49 \\
\hline AK & 13,361 & $28.4 \%$ & $570,640.95$ & 0.02 \\
\hline $\mathrm{AL}$ & 94,797 & $28.5 \%$ & $50,645.33$ & 1.87 \\
\hline$A R$ & 69,287 & $28.6 \%$ & $52,035.48$ & 1.33 \\
\hline $\mathrm{AZ}$ & 116,967 & $28.7 \%$ & $113,594.08$ & 1.03 \\
\hline CA & 468,911 & $28.6 \%$ & $155,779.22$ & 3.01 \\
\hline $\mathrm{CO}$ & 82,982 & $28.5 \%$ & $103,641.89$ & 0.80 \\
\hline $\mathrm{CT}$ & 66,196 & $28.6 \%$ & $4,842.36$ & 13.67 \\
\hline $\mathrm{DC}$ & 8,677 & $27.9 \%$ & 61.05 & 142.13 \\
\hline$\overline{\mathrm{DE}}$ & 23,176 & $28.7 \%$ & $1,948.54$ & 11.89 \\
\hline $\mathrm{FL}$ & 349,349 & $28.6 \%$ & $53,624.76$ & 6.51 \\
\hline GA & 145,585 & $28.5 \%$ & $57,513.49$ & 2.53 \\
\hline $\mathrm{HI}$ & 18,327 & $28.3 \%$ & $6,422.63$ & 2.85 \\
\hline IA & 80,449 & $28.5 \%$ & $55,857.13$ & 1.44 \\
\hline ID & 30,174 & $28.6 \%$ & $82,643.12$ & 0.37 \\
\hline $\mathrm{IL}$ & 240,962 & $28.5 \%$ & $55,518.93$ & 4.34 \\
\hline IN & 125,338 & $28.5 \%$ & $35,826.11$ & 3.50 \\
\hline $\mathrm{KS}$ & 66,086 & $28.5 \%$ & $81,758.72$ & 0.81 \\
\hline $\mathrm{KY}$ & 80,214 & $28.5 \%$ & $39,486.34$ & 2.03 \\
\hline LA & 70,631 & $28.4 \%$ & $43,203.90$ & 1.63 \\
\hline MA & 136,681 & $28.6 \%$ & $7,800.06$ & 17.52 \\
\hline $\mathrm{MD}$ & 112,872 & $28.5 \%$ & $9,707.24$ & 11.63 \\
\hline $\mathrm{ME}$ & 33,960 & $28.6 \%$ & $30,842.92$ & 1.10 \\
\hline $\mathrm{Ml}$ & 159,521 & $28.5 \%$ & $56,538.90$ & 2.82 \\
\hline $\mathrm{MN}$ & 58,704 & $28.4 \%$ & $79,626.74$ & 0.74 \\
\hline $\mathrm{MO}$ & 115,685 & $28.5 \%$ & $68,741.52$ & 1.68 \\
\hline $\mathrm{MS}$ & 63,435 & $28.4 \%$ & $46,923.27$ & 1.35 \\
\hline MT & 28,505 & $28.5 \%$ & $145,545.80$ & 0.20 \\
\hline NC & 174,860 & $28.5 \%$ & $48,617.91$ & 3.60 \\
\hline ND & 16,390 & $28.4 \%$ & $69,000.80$ & 0.24 \\
\hline $\mathrm{NE}$ & 46,227 & $28.5 \%$ & $76,824.17$ & 0.60 \\
\hline $\mathrm{NH}$ & 37,449 & $28.7 \%$ & $8,952.65$ & 4.18 \\
\hline NJ & 155,111 & $28.6 \%$ & $7,354.22$ & 21.09 \\
\hline NM & 36,493 & $28.5 \%$ & $121,298.15$ & 0.30 \\
\hline NV & 44,124 & $28.6 \%$ & $109,781.18$ & 0.40 \\
\hline NY & 240,431 & $28.5 \%$ & $47,126.40$ & 5.10 \\
\hline $\mathrm{OH}$ & 172,428 & $28.5 \%$ & $40,860.69$ & 4.22 \\
\hline $\mathrm{OK}$ & 81,203 & $28.6 \%$ & $68,594.92$ & 1.18 \\
\hline OR & 66,042 & $28.6 \%$ & $95,988.01$ & 0.69 \\
\hline $\mathrm{PA}$ & 208,600 & $28.5 \%$ & $44,742.70$ & 4.66 \\
\hline $\mathrm{PR}$ & 7,601 & $28.9 \%$ & $3,423.78$ & 2.22 \\
\hline $\mathrm{RI}$ & 16,030 & $28.6 \%$ & $1,033.81$ & 15.51 \\
\hline SC & 108,225 & $28.5 \%$ & $30,060.70$ & 3.60 \\
\hline SD & 21,538 & $28.5 \%$ & $75,811.00$ & 0.28 \\
\hline $\mathrm{TN}$ & 115,013 & $28.5 \%$ & $41,234.90$ & 2.79 \\
\hline $\mathrm{TX}$ & 355,280 & $28.6 \%$ & $261,231.71$ & 1.36 \\
\hline UT & 35,516 & $28.6 \%$ & $82,169.62$ & 0.43 \\
\hline VA & 164,566 & $28.5 \%$ & $39,490.09$ & 4.17 \\
\hline $\mathrm{VI}$ & 2,530 & $27.2 \%$ & 134.32 & 18.84 \\
\hline VT & 19,523 & $28.6 \%$ & $9,216.66$ & 2.12 \\
\hline WA & 130,898 & $28.6 \%$ & $66,455.52$ & 1.97 \\
\hline WI & 100,047 & $28.5 \%$ & $54,157.80$ & 1.85 \\
\hline WV & 38,286 & $28.5 \%$ & $24,038.21$ & 1.59 \\
\hline WY & 16,231 & $28.5 \%$ & $97,093.14$ & 0.17 \\
\hline
\end{tabular}


Counties with less than 100 MDPP-Eligible Part B Beneficiaries were excluded. * Proportions of beneficiaries enrolled in Part B (at least one month in Part B) per our study population of Medicare beneficiaries by county. Additionally, readers that are interested in MDPP eligible Part B beneficiaries per 1,000 can multiple the prevalence rates presented in S2 Table by 1,000.

S2 Table. Estimated Number, Prevalence, Density (MDPP-Eligible per Square Mile of Land Area) of MDPP-Eligible Part B Beneficiaries, by County

\begin{tabular}{|c|c|c|c|c|c|c|c|c|}
\hline GEOID & State & County Name & Status & Estimated MDPP eligible & Prevalence & $\begin{array}{l}\text { Land area (square } \\
\text { miles) }\end{array}$ & $\begin{array}{l}\text { Density } \\
\text { (eligible } \\
\text { beneficiaries } \\
\text { per square } \\
\text { mile of land } \\
\text { area) }\end{array}$ & $\begin{array}{l}\text { *Part B } \\
\text { beneficiaries }\end{array}$ \\
\hline 02020 & AK & Anchorage & urban & 5,178 & $28.5 \%$ & $1,704.68$ & 3.038 & $82.6 \%$ \\
\hline 02050 & $A K$ & Bethel & rural & 216 & $28.0 \%$ & $40,570.00$ & 0.005 & $81.7 \%$ \\
\hline 02060 & AK & Bristol Bay & rural & 114 & $27.9 \%$ & 503.84 & 0.225 & $87.0 \%$ \\
\hline 02090 & AK & Fairbanks North Star & urban & 1,512 & $28.4 \%$ & 7338.21 & 0.206 & $86.5 \%$ \\
\hline 02122 & $A K$ & Kenai Peninsula & rural & 1,613 & $28.3 \%$ & 16075.33 & 0.100 & $88.9 \%$ \\
\hline 02130 & $A K$ & Ketchikan Gateway & rural & 378 & $28.1 \%$ & 4858.41 & 0.078 & $88.0 \%$ \\
\hline 02150 & $A K$ & Kodiak Island & rural & 153 & $28.3 \%$ & 6549.58 & 0.023 & $81.4 \%$ \\
\hline 02170 & $A K$ & Matanuska-Susitna & urban & 1,653 & $28.5 \%$ & 24607.9 & 0.067 & $86.1 \%$ \\
\hline 02180 & $A K$ & Nome & rural & 122 & $27.8 \%$ & $22,961.76$ & 0.005 & $74.8 \%$ \\
\hline 02220 & $\mathrm{AK}$ & Sitka & rural & 221 & $28.3 \%$ & $2,870.34$ & 0.077 & $86.1 \%$ \\
\hline 02240 & $\mathrm{AK}$ & Southeast Fairbanks & rural & 178 & $28.0 \%$ & 24768.81 & 0.007 & $85.9 \%$ \\
\hline 02261 & $A K$ & Valdez-Cordova & rural & 207 & $28.0 \%$ & 34239.88 & 0.006 & $86.2 \%$ \\
\hline 02290 & $A K$ & Yukon-Koyukuk & rural & 151 & $28.0 \%$ & 145504.79 & 0.001 & $80.0 \%$ \\
\hline 01005 & $\mathrm{AL}$ & Barbour & rural & 543 & $28.4 \%$ & 884.88 & 0.613 & $73.1 \%$ \\
\hline 01007 & $\mathrm{AL}$ & Bibb & urban & 344 & $28.4 \%$ & 622.58 & 0.552 & $53.8 \%$ \\
\hline 01009 & $\mathrm{AL}$ & Blount & urban & 777 & $28.5 \%$ & 644.78 & 1.205 & $50.1 \%$ \\
\hline 01011 & $\mathrm{AL}$ & Bullock & rural & 113 & $27.6 \%$ & 622.80 & 0.181 & $68.0 \%$ \\
\hline 01013 & $\mathrm{AL}$ & Butler & rural & 442 & $28.1 \%$ & 776.83 & 0.568 & $80.2 \%$ \\
\hline 01015 & $\mathrm{AL}$ & Calhoun & urban & 2,848 & $28.5 \%$ & 605.87 & 4.700 & $75.2 \%$ \\
\hline 01017 & $\mathrm{AL}$ & Chambers & rural & 827 & $28.5 \%$ & 596.53 & 1.386 & $73.4 \%$ \\
\hline 01019 & $\mathrm{AL}$ & Cherokee & rural & 577 & $28.9 \%$ & 553.70 & 1.041 & $68.6 \%$ \\
\hline 01021 & $\mathrm{AL}$ & Chilton & urban & 603 & $28.6 \%$ & 692.85 & 0.870 & $48.7 \%$ \\
\hline 01023 & $\mathrm{AL}$ & Choctaw & rural & 372 & $28.1 \%$ & 913.50 & 0.407 & $86.6 \%$ \\
\hline 01025 & $\overline{A L}$ & Clarke & rural & 613 & $28.2 \%$ & $1,238.46$ & 0.495 & $70.3 \%$ \\
\hline 01027 & $\mathrm{AL}$ & Clay & rural & 390 & $28.5 \%$ & 603.96 & 0.646 & $78.2 \%$ \\
\hline 01029 & $\mathrm{AL}$ & Cleburne & rural & 353 & $28.4 \%$ & 560.10 & 0.631 & $76.4 \%$ \\
\hline 01031 & $\mathrm{AL}$ & Coffee & rural & 1,178 & $28.6 \%$ & 678.97 & 1.735 & $83.1 \%$ \\
\hline 01033 & $\mathrm{AL}$ & Colbert & urban & 1,412 & $28.7 \%$ & 592.62 & 2.382 & $81.3 \%$ \\
\hline 01035 & $\mathrm{AL}$ & Conecuh & rural & 301 & $28.0 \%$ & 850.16 & 0.355 & $79.3 \%$ \\
\hline 01037 & $\mathrm{AL}$ & Coosa & rural & 268 & $28.1 \%$ & 650.93 & 0.412 & $78.0 \%$ \\
\hline 01039 & $\mathrm{AL}$ & Covington & rural & 1,094 & $28.5 \%$ & $1,030.46$ & 1.061 & $83.2 \%$ \\
\hline 01041 & $\mathrm{AL}$ & Crenshaw & rural & 334 & $28.3 \%$ & 608.84 & 0.549 & $72.2 \%$ \\
\hline 01047 & $\mathrm{AL}$ & Dallas & rural & 778 & $28.1 \%$ & 978.69 & 0.795 & $67.1 \%$ \\
\hline 01049 & $\mathrm{AL}$ & DeKalb & rural & 1,499 & $28.5 \%$ & 777.09 & 1.929 & $72.6 \%$ \\
\hline 01051 & $\mathrm{AL}$ & Elmore & urban & 1,628 & $28.6 \%$ & 618.48 & 2.633 & $65.5 \%$ \\
\hline 01053 & $\mathrm{AL}$ & Escambia & rural & 812 & $28.4 \%$ & 945.08 & 0.859 & $76.6 \%$ \\
\hline 01055 & $\mathrm{AL}$ & Etowah & urban & 2,324 & $28.6 \%$ & 534.99 & 4.344 & $69.7 \%$ \\
\hline 01057 & $\mathrm{AL}$ & Fayette & rural & 427 & $28.6 \%$ & 627.66 & 0.681 & $81.1 \%$ \\
\hline 01059 & $\mathrm{AL}$ & Franklin & rural & 834 & $28.7 \%$ & 633.82 & 1.316 & $84.2 \%$ \\
\hline 01061 & $\mathrm{AL}$ & Geneva & urban & 770 & $28.4 \%$ & 574.41 & 1.340 & $81.4 \%$ \\
\hline 01063 & $\mathrm{AL}$ & Greene & rural & 169 & $27.8 \%$ & 647.11 & 0.262 & $82.6 \%$ \\
\hline 01065 & $\mathrm{AL}$ & Hale & urban & 407 & $27.8 \%$ & 643.94 & 0.631 & $84.9 \%$ \\
\hline 01067 & $\mathrm{AL}$ & Henry & urban & 449 & $28.4 \%$ & 561.75 & 0.799 & $69.4 \%$ \\
\hline 01069 & $\mathrm{AL}$ & Houston & urban & 2,359 & $28.6 \%$ & 579.82 & 4.069 & $76.0 \%$ \\
\hline 01071 & $\mathrm{AL}$ & Jackson & rural & 1,391 & $28.7 \%$ & $1,077.87$ & 1.290 & $75.4 \%$ \\
\hline 01073 & $A L$ & Jefferson & urban & 11,310 & $28.5 \%$ & $1,111.28$ & 10.178 & $48.0 \%$ \\
\hline 01075 & $\mathrm{AL}$ & Lamar & rural & 470 & $28.4 \%$ & 604.85 & 0.777 & $86.2 \%$ \\
\hline
\end{tabular}




\begin{tabular}{|c|c|c|c|c|c|c|c|c|}
\hline 01077 & $\mathrm{AL}$ & Lauderdale & urban & 2,633 & $28.5 \%$ & 667.70 & 3.944 & $80.5 \%$ \\
\hline 01079 & $\mathrm{AL}$ & Lawrence & urban & 590 & $28.5 \%$ & 690.68 & 0.854 & $81.4 \%$ \\
\hline 01081 & $\mathrm{AL}$ & Lee & urban & 2,144 & $28.4 \%$ & 607.54 & 3.529 & $75.2 \%$ \\
\hline 01083 & $\mathrm{AL}$ & Limestone & urban & 1,523 & $28.6 \%$ & 559.94 & 2.719 & $73.8 \%$ \\
\hline 01085 & $\mathrm{AL}$ & Lowndes & urban & 152 & $27.6 \%$ & 715.91 & 0.213 & $54.4 \%$ \\
\hline 01087 & $\mathrm{AL}$ & Macon & rural & 387 & $28.0 \%$ & 608.89 & 0.636 & $61.3 \%$ \\
\hline 01089 & $\mathrm{AL}$ & Madison & urban & 7,220 & $28.5 \%$ & 801.59 & 9.007 & $72.5 \%$ \\
\hline 01091 & $\mathrm{AL}$ & Marengo & rural & 406 & $28.3 \%$ & 976.88 & 0.416 & $80.0 \%$ \\
\hline 01093 & $\mathrm{AL}$ & Marion & rural & 807 & $28.6 \%$ & 742.29 & 1.088 & $83.9 \%$ \\
\hline 01095 & $\mathrm{AL}$ & Marshall & rural & 2,450 & $28.8 \%$ & 565.84 & 4.330 & $77.1 \%$ \\
\hline 01099 & $\mathrm{AL}$ & Monroe & rural & 468 & $28.2 \%$ & $1,025.67$ & 0.456 & $76.9 \%$ \\
\hline 01101 & $\mathrm{AL}$ & Montgomery & urban & 3,903 & $28.4 \%$ & 784.25 & 4.976 & $64.0 \%$ \\
\hline 01103 & $\mathrm{AL}$ & Morgan & urban & 2,962 & $28.6 \%$ & 579.34 & 5.113 & $78.6 \%$ \\
\hline 01105 & $\mathrm{AL}$ & Perry & rural & 191 & $27.8 \%$ & 719.66 & 0.266 & $83.0 \%$ \\
\hline 01107 & $\mathrm{AL}$ & Pickens & urban & 487 & $28.2 \%$ & 881.41 & 0.553 & $84.7 \%$ \\
\hline 01109 & $\mathrm{AL}$ & Pike & rural & 643 & $28.4 \%$ & 672.09 & 0.957 & $69.7 \%$ \\
\hline 01111 & $\mathrm{AL}$ & Randolph & rural & 555 & $28.6 \%$ & 580.55 & 0.956 & $77.0 \%$ \\
\hline 01113 & $\mathrm{AL}$ & Russell & urban & 1,024 & $28.5 \%$ & 641.14 & 1.597 & $67.7 \%$ \\
\hline 01117 & $\mathrm{AL}$ & Shelby & urban & 1,894 & $28.7 \%$ & 784.93 & 2.414 & $48.4 \%$ \\
\hline 01121 & $\mathrm{AL}$ & Talladega & rural & 1,564 & $28.5 \%$ & 736.78 & 2.122 & $65.9 \%$ \\
\hline 01123 & $\mathrm{AL}$ & Tallapoosa & rural & 1,116 & $28.3 \%$ & 716.52 & 1.557 & $79.7 \%$ \\
\hline 01125 & $\mathrm{AL}$ & Tuscaloosa & urban & 3,422 & $28.6 \%$ & $1,321.75$ & 2.589 & $78.9 \%$ \\
\hline 01127 & $\mathrm{AL}$ & Walker & urban & 1,456 & $28.7 \%$ & 791.19 & 1.841 & $56.2 \%$ \\
\hline 01129 & $\mathrm{AL}$ & Washington & rural & 426 & $28.4 \%$ & $1,080.21$ & 0.395 & $79.7 \%$ \\
\hline 01131 & $\mathrm{AL}$ & Wilcox & rural & 245 & $27.6 \%$ & 888.50 & 0.275 & $79.1 \%$ \\
\hline 01133 & $\mathrm{AL}$ & Winston & rural & 708 & $28.7 \%$ & 612.98 & 1.155 & $79.2 \%$ \\
\hline 05001 & $\mathrm{AR}$ & Arkansas & rural & 569 & $28.4 \%$ & 988.77 & 0.575 & $82.6 \%$ \\
\hline 05003 & AR & Ashley & rural & 649 & $28.6 \%$ & 925.35 & 0.702 & $89.3 \%$ \\
\hline 05005 & $\mathrm{AR}$ & Baxter & rural & 1,774 & $28.9 \%$ & 554.28 & 3.200 & $67.7 \%$ \\
\hline 05007 & $A R$ & Benton & urban & 3,959 & $28.6 \%$ & 847.36 & 4.673 & $59.1 \%$ \\
\hline 05009 & $A R$ & Boone & rural & 1,179 & $28.6 \%$ & 590.23 & 1.997 & $70.9 \%$ \\
\hline 05011 & $A R$ & Bradley & rural & 314 & $28.5 \%$ & 649.23 & 0.484 & $87.9 \%$ \\
\hline 05013 & $A R$ & Calhoun & rural & 121 & $29.0 \%$ & 628.58 & 0.193 & $82.2 \%$ \\
\hline 05015 & $A R$ & Carroll & rural & 735 & $28.5 \%$ & 630.09 & 1.167 & $62.2 \%$ \\
\hline 05017 & $A R$ & Chicot & rural & 322 & $28.0 \%$ & 644.30 & 0.500 & $83.3 \%$ \\
\hline 05019 & $A R$ & Clark & rural & 615 & $28.5 \%$ & 866.07 & 0.710 & $77.5 \%$ \\
\hline 05021 & $A R$ & Clay & rural & 520 & $28.6 \%$ & 639.46 & 0.813 & $81.4 \%$ \\
\hline 05029 & AR & Conway & rural & 621 & $28.6 \%$ & 552.25 & 1.125 & $75.4 \%$ \\
\hline 05031 & $A R$ & Craighead & urban & 2,196 & $28.8 \%$ & 707.21 & 3.106 & $78.5 \%$ \\
\hline 05033 & $A R$ & Crawford & urban & 1,037 & $28.6 \%$ & 593.09 & 1.748 & $54.0 \%$ \\
\hline 05035 & $\mathrm{AR}$ & Crittenden & urban & 809 & $28.5 \%$ & 609.76 & 1.326 & $73.6 \%$ \\
\hline 05037 & $A R$ & Cross & rural & 446 & $28.6 \%$ & 616.38 & 0.723 & $78.3 \%$ \\
\hline 05039 & AR & Dallas & rural & 192 & $28.2 \%$ & 667.39 & 0.288 & $76.7 \%$ \\
\hline 05041 & AR & Desha & rural & 320 & $28.4 \%$ & 768.15 & 0.416 & $84.4 \%$ \\
\hline 05043 & AR & Drew & rural & 429 & $28.4 \%$ & 828.36 & 0.518 & $84.7 \%$ \\
\hline 05045 & AR & Faulkner & urban & 2,308 & $28.7 \%$ & 647.88 & 3.562 & $81.5 \%$ \\
\hline 05047 & AR & Franklin & rural & 407 & $28.7 \%$ & 608.86 & 0.668 & $64.8 \%$ \\
\hline 05049 & AR & Fulton & rural & 396 & $28.8 \%$ & 618.19 & 0.640 & $72.3 \%$ \\
\hline 05051 & $\mathrm{AR}$ & Garland & urban & 4,270 & $28.8 \%$ & 677.78 & 6.300 & $73.7 \%$ \\
\hline 05053 & AR & Grant & urban & 431 & $28.9 \%$ & 631.81 & 0.682 & $79.9 \%$ \\
\hline 05055 & AR & Greene & rural & 967 & $28.7 \%$ & 577.70 & 1.674 & $74.7 \%$ \\
\hline 05057 & AR & Hempstead & rural & 448 & $28.3 \%$ & 727.52 & 0.616 & $75.1 \%$ \\
\hline 05059 & AR & Hot Spring & rural & 816 & $28.5 \%$ & 615.2 & 1.326 & $77.8 \%$ \\
\hline 05061 & AR & Howard & rural & 390 & $28.8 \%$ & 588.55 & 0.662 & $78.2 \%$ \\
\hline 05063 & AR & Independence & rural & 1,081 & $28.7 \%$ & 763.95 & 1.416 & $86.6 \%$ \\
\hline 05065 & AR & Izard & rural & 474 & $28.8 \%$ & 580.58 & 0.816 & $76.1 \%$ \\
\hline 05067 & AR & Jackson & rural & 432 & $28.3 \%$ & 633.94 & 0.682 & $84.9 \%$ \\
\hline 05069 & AR & Jefferson & urban & 1,515 & $28.2 \%$ & 870.75 & 1.740 & $69.8 \%$ \\
\hline 05071 & AR & Johnson & rural & 527 & $28.8 \%$ & 659.80 & 0.799 & $67.7 \%$ \\
\hline 05073 & AR & Lafayette & rural & 191 & $27.9 \%$ & 528.27 & 0.362 & $83.6 \%$ \\
\hline 05075 & AR & Lawrence & rural & 549 & $28.6 \%$ & 587.61 & 0.935 & $79.1 \%$ \\
\hline 05077 & $A R$ & Lee & rural & 182 & $27.8 \%$ & 602.62 & 0.301 & $70.2 \%$ \\
\hline 05079 & $A R$ & Lincoln & urban & 240 & $28.4 \%$ & 561.52 & 0.427 & $78.1 \%$ \\
\hline 05081 & AR & Little River & urban & 347 & $28.4 \%$ & 532.25 & 0.651 & $77.5 \%$ \\
\hline 05083 & $\mathrm{AR}$ & Logan & rural & 592 & $28.5 \%$ & 708.12 & 0.836 & $69.5 \%$ \\
\hline 05085 & AR & Lonoke & urban & 1,383 & $28.8 \%$ & 770.73 & 1.794 & $77.0 \%$ \\
\hline 05087 & $A R$ & Madison & urban & 427 & $28.7 \%$ & 834.26 & 0.511 & $57.9 \%$ \\
\hline 05089 & $A R$ & Marion & rural & 514 & $28.7 \%$ & 597.01 & 0.861 & $65.3 \%$ \\
\hline 05091 & $A R$ & Miller & urban & 894 & $28.5 \%$ & 625.58 & 1.429 & $72.9 \%$ \\
\hline 05093 & $A R$ & Mississippi & rural & 770 & $28.6 \%$ & 900.57 & 0.855 & $74.3 \%$ \\
\hline 05095 & $A R$ & Monroe & rural & 206 & $28.3 \%$ & 607.12 & 0.339 & $77.6 \%$ \\
\hline 05097 & $A R$ & Montgomery & rural & 309 & $28.5 \%$ & 779.88 & 0.396 & $77.1 \%$ \\
\hline 05099 & $A R$ & Nevada & rural & 245 & $28.3 \%$ & 617.84 & 0.397 & $75.3 \%$ \\
\hline 05101 & $A R$ & Newton & rural & 242 & $28.6 \%$ & 820.90 & 0.295 & $68.6 \%$ \\
\hline
\end{tabular}




\begin{tabular}{|c|c|c|c|c|c|c|c|c|}
\hline 05107 & $A R$ & Phillips & rural & 426 & $28.0 \%$ & 695.66 & 0.612 & $72.8 \%$ \\
\hline 05109 & $\mathrm{AR}$ & Pike & rural & 341 & $28.5 \%$ & 600.62 & 0.568 & $80.6 \%$ \\
\hline 05111 & AR & Poinsett & urban & 610 & $28.7 \%$ & 758.39 & 0.804 & $76.9 \%$ \\
\hline 05113 & AR & Polk & rural & 723 & $28.8 \%$ & 857.68 & 0.843 & $80.8 \%$ \\
\hline 05115 & AR & Pope & rural & 1,359 & $28.6 \%$ & 812.55 & 1.672 & $71.8 \%$ \\
\hline 05117 & AR & Prairie & rural & 267 & $28.6 \%$ & 647.96 & 0.412 & $85.0 \%$ \\
\hline 05119 & $\mathrm{AR}$ & Pulaski & urban & 9,170 & $28.6 \%$ & 759.76 & 12.070 & $72.1 \%$ \\
\hline 05121 & AR & Randolph & rural & 467 & $28.7 \%$ & 652.19 & 0.717 & $71.7 \%$ \\
\hline 05125 & AR & Saline & urban & 1,338 & $28.8 \%$ & 723.60 & 1.849 & $78.0 \%$ \\
\hline 05127 & AR & Scott & rural & 279 & $28.5 \%$ & 892.32 & 0.313 & $66.0 \%$ \\
\hline 05131 & AR & Sebastian & urban & 2,341 & $28.6 \%$ & 531.91 & 4.401 & $61.9 \%$ \\
\hline 05133 & AR & Sevier & rural & 334 & $28.6 \%$ & 565.13 & 0.590 & $80.1 \%$ \\
\hline 05135 & $A R$ & Sharp & rural & 715 & $28.8 \%$ & 604.44 & 1.184 & $75.9 \%$ \\
\hline 05123 & AR & St. Francis & rural & 550 & $28.3 \%$ & 634.77 & 0.866 & $72.5 \%$ \\
\hline 05137 & $A R$ & Stone & rural & 515 & $28.8 \%$ & 606.40 & 0.850 & $85.1 \%$ \\
\hline 05139 & AR & Union & rural & 1,176 & $28.2 \%$ & $1,039.21$ & 1.132 & $83.9 \%$ \\
\hline 05141 & AR & Van Buren & rural & 593 & $28.7 \%$ & 708.14 & 0.837 & $74.1 \%$ \\
\hline 05143 & AR & Washington & urban & 3,706 & $28.7 \%$ & 941.97 & 3.934 & $65.9 \%$ \\
\hline 05145 & AR & White & rural & 2,089 & $28.8 \%$ & $1,035.08$ & 2.018 & $79.1 \%$ \\
\hline 05149 & $A R$ & Yell & rural & 501 & $28.7 \%$ & 929.98 & 0.538 & $74.5 \%$ \\
\hline 04001 & $A Z$ & Apache & rural & 1,225 & $28.2 \%$ & $11,197.52$ & 0.109 & $76.8 \%$ \\
\hline 04003 & $\mathrm{AZ}$ & Cochise & urban & 3,304 & $28.6 \%$ & $6,165.69$ & 0.536 & $61.1 \%$ \\
\hline 04005 & $A Z$ & Coconino & urban & 3,241 & $28.6 \%$ & $18,618.89$ & 0.174 & $77.3 \%$ \\
\hline 04007 & $A Z$ & Gila & rural & 2,172 & $28.6 \%$ & $4,757.93$ & 0.457 & $78.7 \%$ \\
\hline 04009 & $A Z$ & Graham & rural & 560 & $28.4 \%$ & $4,622.60$ & 0.121 & $60.8 \%$ \\
\hline 04011 & $A Z$ & Greenlee & rural & 163 & $28.5 \%$ & $1,843.13$ & 0.088 & $77.7 \%$ \\
\hline 04012 & $A Z$ & La Paz & rural & 671 & $28.6 \%$ & 4499.63 & 0.149 & $74.5 \%$ \\
\hline 04013 & $A Z$ & Maricopa & urban & 58,677 & $28.7 \%$ & $9,200.14$ & 6.378 & $48.9 \%$ \\
\hline 04015 & $A Z$ & Mohave & urban & 6,764 & $28.6 \%$ & $13,311.08$ & 0.508 & $66.7 \%$ \\
\hline 04017 & $A Z$ & Navajo & rural & 2,483 & $28.6 \%$ & $9,950.42$ & 0.249 & $70.8 \%$ \\
\hline 04019 & $A Z$ & Pima & urban & 18,831 & $28.7 \%$ & $9,187.04$ & 2.050 & $46.8 \%$ \\
\hline 04021 & $A Z$ & Pinal & urban & 5,609 & $28.7 \%$ & $5,365.61$ & 1.045 & $51.1 \%$ \\
\hline 04023 & $A Z$ & Santa Cruz & rural & 786 & $28.6 \%$ & 1236.92 & 0.636 & $42.4 \%$ \\
\hline 04025 & $A Z$ & Yavapai & urban & 8,662 & $28.7 \%$ & $8,123.50$ & 1.066 & $65.7 \%$ \\
\hline 04027 & $A Z$ & Yuma & urban & 3,818 & $28.8 \%$ & $5,513.99$ & 0.692 & $73.7 \%$ \\
\hline 06001 & CA & Alameda & urban & 16,633 & $28.4 \%$ & 739.02 & 22.507 & $38.0 \%$ \\
\hline 06005 & CA & Amador & rural & 1,430 & $28.6 \%$ & 594.58 & 2.405 & $69.5 \%$ \\
\hline 06007 & CA & Butte & urban & 7,184 & $28.7 \%$ & $1,636.46$ & 4.390 & $91.2 \%$ \\
\hline 06015 & CA & Del Norte & rural & 764 & $28.5 \%$ & 1006.37 & 0.759 & $83.7 \%$ \\
\hline 06017 & CA & El Dorado & urban & 4,353 & $28.6 \%$ & 1707.88 & 2.549 & $54.9 \%$ \\
\hline 06019 & CA & Fresno & urban & 11,428 & $28.5 \%$ & $5,957.99$ & 1.918 & $53.2 \%$ \\
\hline 06021 & CA & Glenn & rural & 708 & $28.3 \%$ & $1,313.95$ & 0.539 & $91.0 \%$ \\
\hline 06023 & CA & Humboldt & rural & 4,072 & $28.6 \%$ & $3,567.99$ & 1.141 & $88.7 \%$ \\
\hline 06025 & CA & Imperial & urban & 3,030 & $28.3 \%$ & $4,176.60$ & 0.725 & $78.5 \%$ \\
\hline 06027 & CA & Inyo & rural & 525 & $28.5 \%$ & $10,180.88$ & 0.052 & $89.8 \%$ \\
\hline 06029 & CA & Kern & urban & 7,880 & $28.6 \%$ & $8,131.92$ & 0.969 & $45.3 \%$ \\
\hline 06031 & CA & Kings & urban & 1,714 & $28.6 \%$ & $1,389.42$ & 1.234 & $73.1 \%$ \\
\hline 06033 & CA & Lake & rural & 2,325 & $28.5 \%$ & $1,256.46$ & 1.850 & $85.8 \%$ \\
\hline 06035 & CA & Lassen & rural & 631 & $28.6 \%$ & $4,541.18$ & 0.139 & $86.5 \%$ \\
\hline 06037 & CA & Los Angeles & urban & 90,047 & $28.7 \%$ & 4057.88 & 22.191 & $35.7 \%$ \\
\hline 06039 & CA & Madera & urban & 2,032 & $28.4 \%$ & $2,137.07$ & 0.951 & $50.0 \%$ \\
\hline 06041 & CA & Marin & urban & 6,092 & $28.7 \%$ & 520.31 & 11.708 & $49.3 \%$ \\
\hline 06043 & CA & Mariposa & rural & 691 & $28.5 \%$ & $1,448.82$ & 0.477 & $86.1 \%$ \\
\hline 06045 & CA & Mendocino & rural & 3,259 & $28.6 \%$ & $3,506.34$ & 0.929 & $84.6 \%$ \\
\hline 06047 & CA & Merced & urban & 3,597 & $28.4 \%$ & $1,934.97$ & 1.859 & $78.7 \%$ \\
\hline 06049 & CA & Modoc & rural & 329 & $28.6 \%$ & $3,917.77$ & 0.084 & $84.4 \%$ \\
\hline 06051 & CA & Mono & rural & 508 & $28.5 \%$ & $3,048.98$ & 0.166 & $88.3 \%$ \\
\hline 06053 & CA & Monterey & urban & 8,457 & $28.5 \%$ & $3,280.60$ & 2.578 & $85.7 \%$ \\
\hline 06055 & CA & Napa & urban & 2,796 & $28.6 \%$ & 748.36 & 3.736 & $49.0 \%$ \\
\hline 06057 & CA & Nevada & rural & 4,064 & $28.6 \%$ & 957.77 & 4.243 & $78.2 \%$ \\
\hline 06059 & CA & Orange & urban & 34,847 & $28.6 \%$ & 790.57 & 44.078 & $37.7 \%$ \\
\hline 06061 & CA & Placer & urban & 6,802 & $28.7 \%$ & $1,407.01$ & 4.834 & $41.9 \%$ \\
\hline 06063 & CA & Plumas & rural & 947 & $28.6 \%$ & $2,553.04$ & 0.371 & $85.4 \%$ \\
\hline 06065 & CA & Riverside & urban & 20,664 & $28.7 \%$ & $7,206.48$ & 2.867 & $31.6 \%$ \\
\hline 06067 & CA & Sacramento & urban & 16,067 & $28.5 \%$ & 964.64 & 16.655 & $37.2 \%$ \\
\hline 06069 & CA & San Benito & urban & 1,045 & $28.7 \%$ & 1388.71 & 0.752 & $80.8 \%$ \\
\hline 06071 & CA & San Bernardino & urban & 13,394 & $28.6 \%$ & 20056.94 & 0.668 & $27.0 \%$ \\
\hline 06073 & CA & San Diego & urban & 37,900 & $28.6 \%$ & 4206.63 & 9.010 & $39.0 \%$ \\
\hline 06075 & CA & San Francisco & urban & 11,820 & $28.4 \%$ & 46.87 & 252.192 & $43.0 \%$ \\
\hline 06077 & CA & San Joaquin & urban & 8,142 & $28.4 \%$ & 1391.32 & 5.852 & $47.1 \%$ \\
\hline 06079 & CA & San Luis Obispo & urban & 8,454 & $28.6 \%$ & 3298.57 & 2.563 & $79.1 \%$ \\
\hline 06081 & CA & San Mateo & urban & 10,693 & $28.7 \%$ & 448.41 & 23.846 & $42.3 \%$ \\
\hline 06083 & CA & Santa Barbara & urban & 9,452 & $28.5 \%$ & 2735.09 & 3.456 & $72.7 \%$ \\
\hline 06085 & CA & Santa Clara & urban & 21,664 & $28.5 \%$ & 1290.1 & 16.793 & $42.9 \%$ \\
\hline 06087 & CA & Santa Cruz & urban & 6,842 & $28.5 \%$ & 445.17 & 15.370 & $79.6 \%$ \\
\hline
\end{tabular}




\begin{tabular}{|c|c|c|c|c|c|c|c|c|}
\hline 06093 & CA & Siskiyou & rural & 1,982 & $28.6 \%$ & $6,277.89$ & 0.316 & $84.5 \%$ \\
\hline 06095 & CA & Solano & urban & 5,356 & $28.4 \%$ & 821.76 & 6.517 & $40.1 \%$ \\
\hline 06097 & $C A$ & Sonoma & urban & 9,156 & $28.6 \%$ & $1,575.85$ & 5.810 & $44.9 \%$ \\
\hline 06099 & CA & Stanislaus & urban & 5,513 & $28.6 \%$ & $1,494.83$ & 3.688 & $39.9 \%$ \\
\hline 06101 & CA & Sutter & urban & 2,120 & $28.6 \%$ & 602.41 & 3.519 & $88.0 \%$ \\
\hline 06103 & CA & Tehama & rural & 1,807 & $28.6 \%$ & $2,949.71$ & 0.612 & $91.4 \%$ \\
\hline 06105 & $\mathrm{CA}$ & Trinity & rural & 1,055 & $28.5 \%$ & $3,179.25$ & 0.332 & $87.5 \%$ \\
\hline 06107 & CA & Tulare & urban & 6,363 & $28.5 \%$ & $4,824.21$ & 1.319 & $75.0 \%$ \\
\hline 06109 & CA & Tuolumne & rural & 2,464 & $28.6 \%$ & $2,220.88$ & 1.110 & $88.4 \%$ \\
\hline 06111 & $C A$ & Ventura & urban & 13,535 & $28.6 \%$ & $1,843.13$ & 7.344 & $53.5 \%$ \\
\hline 06113 & CA & Yolo & urban & 2,535 & $28.6 \%$ & $1,014.69$ & 2.498 & $45.0 \%$ \\
\hline 06115 & CA & Yuba & urban & 1,316 & $28.6 \%$ & 631.84 & 2.082 & $80.5 \%$ \\
\hline 08001 & $\mathrm{CO}$ & Adams & urban & 3,428 & $28.5 \%$ & $1,167.65$ & 2.936 & $31.9 \%$ \\
\hline 08003 & $\mathrm{CO}$ & Alamosa & rural & 317 & $28.2 \%$ & 722.64 & 0.439 & $66.7 \%$ \\
\hline 08005 & $\mathrm{CO}$ & Arapahoe & urban & 8,553 & $28.6 \%$ & 798.10 & 10.717 & $42.2 \%$ \\
\hline 08007 & $\mathrm{CO}$ & Archuleta & rural & 538 & $28.3 \%$ & $1,350.18$ & 0.398 & $79.2 \%$ \\
\hline 08009 & $\mathrm{CO}$ & Baca & rural & 170 & $28.6 \%$ & $2,554.97$ & 0.067 & $94.3 \%$ \\
\hline 08011 & $\mathrm{CO}$ & Bent & rural & 122 & $28.6 \%$ & $1,512.86$ & 0.081 & $79.9 \%$ \\
\hline 08013 & $\mathrm{CO}$ & Boulder & urban & 5,280 & $28.5 \%$ & 726.29 & 7.270 & $48.6 \%$ \\
\hline 08014 & $\mathrm{CO}$ & Broomfield & urban & 946 & $28.8 \%$ & 33.03 & 28.648 & $38.0 \%$ \\
\hline 08015 & $\mathrm{CO}$ & Chaffee & rural & 778 & $28.7 \%$ & $1,013.40$ & 0.768 & $75.6 \%$ \\
\hline 08019 & $\mathrm{CO}$ & Clear Creek & urban & 121 & $28.2 \%$ & 395.23 & 0.307 & $55.3 \%$ \\
\hline 08021 & $\mathrm{CO}$ & Conejos & rural & 156 & $27.6 \%$ & $1,287.39$ & 0.121 & $59.6 \%$ \\
\hline 08023 & $\mathrm{CO}$ & Costilla & rural & 123 & $28.9 \%$ & $1,226.95$ & 0.100 & $62.0 \%$ \\
\hline 08027 & $\mathrm{CO}$ & Custer & rural & 222 & $28.3 \%$ & 738.63 & 0.301 & $73.1 \%$ \\
\hline 08029 & $\mathrm{CO}$ & Delta & rural & 1,204 & $28.5 \%$ & $1,142.05$ & 1.054 & $69.5 \%$ \\
\hline 08031 & $\mathrm{CO}$ & Denver & urban & 6,837 & $28.4 \%$ & 153.00 & 44.686 & $39.3 \%$ \\
\hline 08035 & $\mathrm{CO}$ & Douglas & urban & 2,440 & $28.6 \%$ & 840.25 & 2.904 & $48.5 \%$ \\
\hline 08037 & $\mathrm{CO}$ & Eagle & rural & 884 & $28.5 \%$ & $1,684.53$ & 0.525 & $83.1 \%$ \\
\hline 08041 & $\mathrm{CO}$ & El Paso & urban & 10,455 & $28.5 \%$ & 2126.8 & 4.916 & $59.1 \%$ \\
\hline 08039 & $\mathrm{CO}$ & Elbert & urban & 502 & $28.3 \%$ & $1,850.85$ & 0.271 & $58.1 \%$ \\
\hline 08043 & $\mathrm{CO}$ & Fremont & rural & 1,267 & $28.5 \%$ & $1,533.07$ & 0.826 & $59.9 \%$ \\
\hline 08045 & $\mathrm{CO}$ & Garfield & rural & 1,323 & $28.6 \%$ & $2,947.56$ & 0.449 & $80.2 \%$ \\
\hline 08049 & $\mathrm{CO}$ & Grand & rural & 374 & $28.6 \%$ & $1,846.33$ & 0.203 & $76.9 \%$ \\
\hline 08051 & $\mathrm{CO}$ & Gunnison & rural & 375 & $28.6 \%$ & $3,239.10$ & 0.116 & $88.1 \%$ \\
\hline 08055 & $\mathrm{CO}$ & Huerfano & rural & 288 & $28.4 \%$ & $1,591.00$ & 0.181 & $75.8 \%$ \\
\hline 08059 & $\mathrm{CO}$ & Jefferson & urban & 6,757 & $28.5 \%$ & 764.21 & 8.842 & $32.5 \%$ \\
\hline 08063 & $\mathrm{CO}$ & Kit Carson & rural & 250 & $28.7 \%$ & 2160.82 & 0.116 & $90.7 \%$ \\
\hline 08067 & $\mathrm{CO}$ & La Plata & rural & 1,569 & $28.6 \%$ & 1692.08 & 0.927 & $81.1 \%$ \\
\hline 08065 & $\mathrm{CO}$ & Lake & rural & 136 & $28.8 \%$ & 376.91 & 0.360 & $81.2 \%$ \\
\hline 08069 & $\mathrm{CO}$ & Larimer & urban & 6,804 & $28.6 \%$ & $2,596.00$ & 2.621 & $61.9 \%$ \\
\hline 08071 & $\mathrm{CO}$ & Las Animas & rural & 470 & $28.3 \%$ & 4772.67 & 0.098 & $73.5 \%$ \\
\hline 08073 & $\mathrm{CO}$ & Lincoln & rural & 157 & $28.5 \%$ & $2,577.63$ & 0.061 & $89.5 \%$ \\
\hline 08075 & $\mathrm{CO}$ & Logan & rural & 565 & $28.5 \%$ & $1,838.55$ & 0.307 & $81.6 \%$ \\
\hline 08077 & $\mathrm{CO}$ & Mesa & urban & 3,288 & $28.6 \%$ & $3,328.97$ & 0.988 & $55.4 \%$ \\
\hline 08081 & $\mathrm{CO}$ & Moffat & rural & 311 & $28.7 \%$ & $4,743.29$ & 0.066 & $81.7 \%$ \\
\hline 08083 & $\mathrm{CO}$ & Montezuma & rural & 911 & $28.5 \%$ & $2,029.53$ & 0.449 & $79.7 \%$ \\
\hline 08085 & $\mathrm{CO}$ & Montrose & rural & 1,423 & $28.7 \%$ & $2,240.70$ & 0.635 & $71.4 \%$ \\
\hline 08087 & $\mathrm{CO}$ & Morgan & rural & 696 & $28.5 \%$ & $1,280.43$ & 0.543 & $82.4 \%$ \\
\hline 08089 & $\mathrm{CO}$ & Otero & rural & 549 & $28.3 \%$ & $1,261.96$ & 0.435 & $73.9 \%$ \\
\hline 08091 & $\mathrm{CO}$ & Ouray & rural & 192 & $28.7 \%$ & 541.59 & 0.354 & $80.1 \%$ \\
\hline 08093 & $\mathrm{CO}$ & Park & urban & 357 & $28.3 \%$ & $2,193.85$ & 0.163 & $61.2 \%$ \\
\hline 08095 & $\mathrm{CO}$ & Phillips & rural & 168 & $28.4 \%$ & 687.93 & 0.244 & $89.4 \%$ \\
\hline 08097 & $\mathrm{CO}$ & Pitkin & rural & 488 & $28.6 \%$ & 970.70 & 0.503 & $88.1 \%$ \\
\hline 08099 & $\mathrm{CO}$ & Prowers & rural & 350 & $28.7 \%$ & $1,638.39$ & 0.214 & $89.2 \%$ \\
\hline 08101 & $\mathrm{CO}$ & Pueblo & urban & 3,138 & $28.6 \%$ & $2,386.10$ & 1.315 & $53.4 \%$ \\
\hline 08103 & $\mathrm{CO}$ & Rio Blanco & rural & 147 & $28.3 \%$ & 3220.93 & 0.046 & $76.3 \%$ \\
\hline 08105 & $\mathrm{CO}$ & Rio Grande & rural & 354 & $28.7 \%$ & 911.96 & 0.388 & $72.7 \%$ \\
\hline 08107 & $\mathrm{CO}$ & Routt & rural & 600 & $28.5 \%$ & $2,362.03$ & 0.254 & $84.6 \%$ \\
\hline 08109 & $\mathrm{CO}$ & Saguache & rural & 187 & $28.5 \%$ & $3,168.52$ & 0.059 & $72.2 \%$ \\
\hline 08113 & $\mathrm{CO}$ & San Miguel & rural & 156 & $28.2 \%$ & 1286.61 & 0.122 & $79.7 \%$ \\
\hline 08115 & $\mathrm{CO}$ & Sedgwick & rural & 107 & $28.5 \%$ & 548.04 & 0.196 & $88.5 \%$ \\
\hline 08117 & $\mathrm{CO}$ & Summit & rural & 574 & $28.4 \%$ & 608.36 & 0.943 & $81.2 \%$ \\
\hline 08119 & $\mathrm{CO}$ & Teller & urban & 605 & $28.4 \%$ & 557.06 & 1.086 & $55.8 \%$ \\
\hline 08121 & $\mathrm{CO}$ & Washington & rural & 161 & $28.6 \%$ & $2,518.03$ & 0.064 & $89.0 \%$ \\
\hline 08123 & $\mathrm{CO}$ & Weld & urban & 3,973 & $28.5 \%$ & $3,987.24$ & 0.996 & $58.7 \%$ \\
\hline 08125 & $\mathrm{CO}$ & Yuma & rural & 327 & $28.4 \%$ & $2,364.40$ & 0.138 & $91.5 \%$ \\
\hline 09001 & CT & Fairfield & urban & 16,894 & $28.6 \%$ & 624.89 & 27.035 & $61.8 \%$ \\
\hline 09003 & CT & Hartford & urban & 15,877 & $28.5 \%$ & 735.10 & 21.599 & $55.4 \%$ \\
\hline 09005 & CT & Litchfield & rural & 4,814 & $28.6 \%$ & 920.56 & 5.229 & $65.1 \%$ \\
\hline 09007 & CT & Middlesex & urban & 3,724 & $28.6 \%$ & 369.30 & 10.084 & $59.4 \%$ \\
\hline 09009 & CT & New Haven & urban & 14,128 & $28.5 \%$ & 604.51 & 23.371 & $53.0 \%$ \\
\hline 09011 & CT & New London & urban & 5,983 & $28.6 \%$ & 664.88 & 8.999 & $66.5 \%$ \\
\hline 09013 & CT & Tolland & urban & 2,519 & $28.7 \%$ & 410.21 & 6.141 & $57.1 \%$ \\
\hline 09015 & CT & Windham & urban & 2,257 & $28.6 \%$ & 512.91 & 4.401 & $62.4 \%$ \\
\hline 11001 & $\mathrm{DC}$ & District of Columbia & urban & 8,677 & $27.9 \%$ & 61.05 & 142.125 & $63.2 \%$ \\
\hline 10001 & $\mathrm{DE}$ & Kent & urban & 3,715 & $28.5 \%$ & 586.18 & 6.337 & $83.9 \%$ \\
\hline 10003 & $\mathrm{DE}$ & New Castle & urban & 10,650 & $28.6 \%$ & 426.29 & 24.984 & $78.0 \%$ \\
\hline 10005 & $\mathrm{DE}$ & Sussex & urban & 8,810 & $28.8 \%$ & 936.08 & 9.412 & $87.5 \%$ \\
\hline 12001 & $\mathrm{FL}$ & Alachua & urban & 4,807 & $28.5 \%$ & 875.02 & 5.494 & $66.5 \%$ \\
\hline 12003 & $\mathrm{FL}$ & Baker & urban & 344 & $28.7 \%$ & 585.23 & 0.587 & $60.0 \%$ \\
\hline 12005 & $\mathrm{FL}$ & Bay & urban & 4,113 & $28.6 \%$ & 758.46 & 5.423 & $71.6 \%$ \\
\hline
\end{tabular}




\begin{tabular}{|c|c|c|c|c|c|c|c|c|}
\hline 12007 & $\mathrm{FL}$ & Bradford & rural & 448 & $28.5 \%$ & 293.96 & 1.524 & $67.2 \%$ \\
\hline 12009 & $\mathrm{FL}$ & Brevard & urban & 13,014 & $28.6 \%$ & $1,015.66$ & 12.814 & $52.3 \%$ \\
\hline 12011 & $\mathrm{FL}$ & Broward & urban & 17,971 & $28.5 \%$ & $1,209.79$ & 14.855 & $32.1 \%$ \\
\hline 12013 & $\mathrm{FL}$ & Calhoun & rural & 244 & $28.6 \%$ & 567.33 & 0.431 & $63.4 \%$ \\
\hline 12015 & $\mathrm{FL}$ & Charlotte & urban & 6,041 & $28.7 \%$ & 680.28 & 8.880 & $60.6 \%$ \\
\hline 12017 & $\mathrm{FL}$ & Citrus & urban & 5,024 & $28.8 \%$ & 581.70 & 8.637 & $60.3 \%$ \\
\hline 12019 & $\mathrm{FL}$ & Clay & urban & 3,457 & $28.6 \%$ & 604.36 & 5.719 & $66.8 \%$ \\
\hline 12021 & $\mathrm{FL}$ & Collier & urban & 12,187 & $28.8 \%$ & $1,998.32$ & 6.099 & $71.7 \%$ \\
\hline 12023 & $\mathrm{FL}$ & Columbia & rural & 1,490 & $28.5 \%$ & 797.57 & 1.868 & $66.8 \%$ \\
\hline 12027 & $\mathrm{FL}$ & DeSoto & rural & 669 & $28.6 \%$ & 637.06 & 1.050 & $66.7 \%$ \\
\hline 12031 & $\mathrm{FL}$ & Duval & urban & 13,468 & $28.5 \%$ & 762.19 & 17.670 & $57.6 \%$ \\
\hline 12033 & $\mathrm{FL}$ & Escambia & urban & 6,252 & $28.4 \%$ & 656.46 & 9.525 & $62.3 \%$ \\
\hline 12035 & $\mathrm{FL}$ & Flagler & urban & 2,842 & $28.6 \%$ & 485.46 & 5.854 & $50.2 \%$ \\
\hline 12037 & $\mathrm{FL}$ & Franklin & rural & 290 & $28.3 \%$ & 534.72 & 0.543 & $64.4 \%$ \\
\hline 12039 & $\mathrm{FL}$ & Gadsden & urban & 487 & $28.0 \%$ & 516.33 & 0.942 & $33.2 \%$ \\
\hline 12041 & $\mathrm{FL}$ & Gilchrist & urban & 384 & $28.6 \%$ & 349.68 & 1.099 & $66.8 \%$ \\
\hline 12043 & $\mathrm{FL}$ & Glades & rural & 137 & $28.3 \%$ & 806.01 & 0.170 & $61.9 \%$ \\
\hline 12045 & $\mathrm{FL}$ & Gulf & urban & 427 & $28.6 \%$ & 564.01 & 0.757 & $73.7 \%$ \\
\hline 12047 & $\mathrm{FL}$ & Hamilton & rural & 282 & $28.0 \%$ & 513.79 & 0.549 & $68.6 \%$ \\
\hline 12053 & $\mathrm{FL}$ & Hernando & urban & 3,597 & $28.6 \%$ & 472.54 & 7.611 & $37.3 \%$ \\
\hline 12055 & $\mathrm{FL}$ & Highlands & urban & 3,266 & $28.8 \%$ & $1,016.61$ & 3.213 & $62.0 \%$ \\
\hline 12057 & $\mathrm{FL}$ & Hillsborough & urban & 14,864 & $28.5 \%$ & $1,020.21$ & 14.570 & $40.6 \%$ \\
\hline 12059 & $\mathrm{FL}$ & Holmes & rural & 474 & $28.7 \%$ & 478.78 & 0.991 & $75.1 \%$ \\
\hline 12061 & $\mathrm{FL}$ & Indian River & urban & 5,974 & $28.7 \%$ & 502.87 & 11.880 & $70.4 \%$ \\
\hline 12063 & $\mathrm{FL}$ & Jackson & rural & 1,141 & $28.5 \%$ & 917.76 & 1.244 & $72.2 \%$ \\
\hline 12065 & $\mathrm{FL}$ & Jefferson & urban & 231 & $28.1 \%$ & 598.10 & 0.386 & $40.3 \%$ \\
\hline 12067 & $\mathrm{FL}$ & Lafayette & rural & 112 & $28.7 \%$ & 543.41 & 0.206 & $74.3 \%$ \\
\hline 12069 & $\mathrm{FL}$ & Lake & urban & 15,067 & $28.9 \%$ & 938.38 & 16.056 & $60.4 \%$ \\
\hline 12071 & $\mathrm{FL}$ & Lee & urban & 18,585 & $28.7 \%$ & 784.51 & 23.690 & $59.6 \%$ \\
\hline 12073 & $\mathrm{FL}$ & Leon & urban & 2,686 & $28.3 \%$ & 666.85 & 4.028 & $36.6 \%$ \\
\hline 12075 & $\mathrm{FL}$ & Levy & rural & 1,020 & $28.6 \%$ & $1,118.21$ & 0.912 & $63.8 \%$ \\
\hline 12079 & $\mathrm{FL}$ & Madison & rural & 395 & $28.4 \%$ & 695.95 & 0.568 & $64.5 \%$ \\
\hline 12081 & $\mathrm{FL}$ & Manatee & urban & 8,151 & $28.6 \%$ & 742.93 & 10.972 & $56.1 \%$ \\
\hline 12083 & $\mathrm{FL}$ & Marion & urban & 8,975 & $28.7 \%$ & $1,584.55$ & 5.664 & $51.0 \%$ \\
\hline 12085 & $\mathrm{FL}$ & Martin & urban & 5,687 & $28.8 \%$ & 543.46 & 10.464 & $67.3 \%$ \\
\hline 12086 & $\mathrm{FL}$ & Miami-Dade & urban & 19,525 & $28.7 \%$ & 1897.72 & 10.288 & $23.8 \%$ \\
\hline 12087 & $\mathrm{FL}$ & Monroe & rural & 2,416 & $28.3 \%$ & 983.28 & 2.457 & $78.9 \%$ \\
\hline 12095 & $\mathrm{FL}$ & Orange & urban & 12,023 & $28.5 \%$ & 903.43 & 13.308 & $43.6 \%$ \\
\hline 12097 & $\mathrm{FL}$ & Osceola & urban & 3,172 & $28.6 \%$ & $1,327.45$ & 2.389 & $35.7 \%$ \\
\hline 12099 & $\mathrm{FL}$ & Palm Beach & urban & 26,652 & $28.7 \%$ & 1969.76 & 13.530 & $49.3 \%$ \\
\hline 12101 & $\mathrm{FL}$ & Pasco & urban & 7,386 & $28.7 \%$ & 746.89 & 9.889 & $36.4 \%$ \\
\hline 12103 & $\mathrm{FL}$ & Pinellas & urban & 17,727 & $28.5 \%$ & 273.80 & 64.746 & $41.9 \%$ \\
\hline 12105 & $\mathrm{FL}$ & Polk & urban & 9,232 & $28.6 \%$ & $1,797.84$ & 5.135 & $43.3 \%$ \\
\hline 12107 & $\mathrm{FL}$ & Putnam & rural & 1,607 & $28.7 \%$ & 727.62 & 2.209 & $61.5 \%$ \\
\hline 12113 & $\mathrm{FL}$ & Santa Rosa & urban & 3,193 & $28.7 \%$ & 1011.6 & 3.156 & $62.8 \%$ \\
\hline 12115 & $\mathrm{FL}$ & Sarasota & urban & 18,349 & $28.8 \%$ & 555.87 & 33.009 & $66.8 \%$ \\
\hline 12117 & $\mathrm{FL}$ & Seminole & urban & 5,519 & $28.6 \%$ & 309.22 & 17.848 & $48.1 \%$ \\
\hline 12109 & $\mathrm{FL}$ & St. Johns & urban & 6,172 & $28.7 \%$ & 600.66 & 10.275 & $67.0 \%$ \\
\hline 12111 & $\mathrm{FL}$ & St. Lucie & urban & 5,910 & $28.6 \%$ & 571.93 & 10.333 & $52.0 \%$ \\
\hline 12119 & $\mathrm{FL}$ & Sumter & urban & 2,148 & $28.6 \%$ & 546.93 & 3.927 & $56.8 \%$ \\
\hline 12121 & $\mathrm{FL}$ & Suwannee & rural & 1,089 & $28.6 \%$ & 688.55 & 1.582 & $69.6 \%$ \\
\hline 12123 & $\mathrm{FL}$ & Taylor & rural & 463 & $28.5 \%$ & $1,043.31$ & 0.444 & $70.1 \%$ \\
\hline 12125 & $\mathrm{FL}$ & Union & rural & 185 & $28.5 \%$ & 243.56 & 0.761 & $67.3 \%$ \\
\hline 12127 & $\mathrm{FL}$ & Volusia & urban & 10,711 & $28.6 \%$ & $1,101.03$ & 9.728 & $43.3 \%$ \\
\hline 12129 & $\mathrm{FL}$ & Wakulla & urban & 370 & $28.4 \%$ & 606.42 & 0.610 & $33.7 \%$ \\
\hline 12131 & $\mathrm{FL}$ & Walton & urban & 1,310 & $28.6 \%$ & $1,037.62$ & 1.262 & $71.3 \%$ \\
\hline 12133 & $\mathrm{FL}$ & Washington & rural & 524 & $28.7 \%$ & 582.80 & 0.899 & $75.5 \%$ \\
\hline 13001 & GA & Appling & rural & 334 & $28.5 \%$ & 507.08 & 0.658 & $66.3 \%$ \\
\hline 13003 & GA & Atkinson & rural & 130 & $28.3 \%$ & 339.38 & 0.384 & $63.9 \%$ \\
\hline 13005 & GA & Bacon & rural & 165 & $28.4 \%$ & 258.58 & 0.637 & $64.4 \%$ \\
\hline 13009 & GA & Baldwin & rural & 571 & $28.6 \%$ & 257.84 & 2.215 & $47.7 \%$ \\
\hline 13011 & GA & Banks & rural & 231 & $28.9 \%$ & 232.09 & 0.995 & $62.3 \%$ \\
\hline 13013 & GA & Barrow & urban & 1,038 & $28.6 \%$ & 160.31 & 6.474 & $56.2 \%$ \\
\hline 13015 & GA & Bartow & urban & 1,564 & $28.6 \%$ & 459.54 & 3.402 & $61.5 \%$ \\
\hline 13017 & GA & Ben Hill & rural & 342 & $28.4 \%$ & 250.12 & 1.366 & $64.2 \%$ \\
\hline 13019 & GA & Berrien & rural & 346 & $28.6 \%$ & 451.90 & 0.767 & $64.5 \%$ \\
\hline 13021 & GA & Bibb & urban & 2,752 & $28.3 \%$ & 249.76 & 11.020 & $58.6 \%$ \\
\hline 13023 & GA & Bleckley & rural & 103 & $28.3 \%$ & 215.87 & 0.475 & $77.0 \%$ \\
\hline 13025 & GA & Brantley & urban & 259 & $28.4 \%$ & 442.36 & 0.585 & $65.6 \%$ \\
\hline 13027 & GA & Brooks & urban & 215 & $28.2 \%$ & 493.05 & 0.436 & $63.3 \%$ \\
\hline 13029 & GA & Bryan & urban & 491 & $28.6 \%$ & 435.97 & 1.127 & $60.1 \%$ \\
\hline 13031 & GA & Bulloch & rural & 877 & $28.6 \%$ & 672.81 & 1.304 & $65.8 \%$ \\
\hline 13033 & GA & Burke & urban & 303 & $28.5 \%$ & 826.97 & 0.367 & $55.1 \%$ \\
\hline 13035 & GA & Butts & urban & 400 & $28.3 \%$ & 184.39 & 2.167 & $53.3 \%$ \\
\hline
\end{tabular}




\begin{tabular}{|c|c|c|c|c|c|c|c|c|}
\hline 13043 & GA & Candler & rural & 167 & $28.0 \%$ & 243.04 & 0.687 & $59.1 \%$ \\
\hline 13045 & GA & Carroll & urban & 1,932 & $28.6 \%$ & 499.08 & 3.872 & $58.4 \%$ \\
\hline 13047 & GA & Catoosa & urban & 934 & $28.6 \%$ & 162.16 & 5.757 & $61.1 \%$ \\
\hline 13049 & GA & Charlton & rural & 170 & $28.6 \%$ & 773.58 & 0.220 & $69.5 \%$ \\
\hline 13051 & GA & Chatham & urban & 4,571 & $28.4 \%$ & 426.44 & 10.719 & $58.8 \%$ \\
\hline 13055 & GA & Chattooga & rural & 517 & $28.6 \%$ & 313.34 & 1.649 & $61.1 \%$ \\
\hline 13057 & GA & Cherokee & urban & 2,100 & $28.8 \%$ & 421.67 & 4.980 & $56.0 \%$ \\
\hline 13059 & GA & Clarke & urban & 1,802 & $28.5 \%$ & 119.20 & 15.119 & $65.0 \%$ \\
\hline 13063 & GA & Clayton & urban & 2,859 & $28.5 \%$ & 141.57 & 20.196 & $46.1 \%$ \\
\hline 13065 & GA & Clinch & rural & 114 & $28.0 \%$ & 800.22 & 0.142 & $64.3 \%$ \\
\hline 13067 & GA & Cobb & urban & 11,991 & $28.6 \%$ & 339.55 & 35.313 & $54.7 \%$ \\
\hline 13069 & GA & Coffee & rural & 594 & $28.3 \%$ & 575.10 & 1.034 & $64.0 \%$ \\
\hline 13071 & GA & Colquitt & rural & 788 & $28.6 \%$ & 544.15 & 1.449 & $66.0 \%$ \\
\hline 13073 & $\mathrm{GA}$ & Columbia & urban & 1,825 & $28.5 \%$ & 290.09 & 6.292 & $63.1 \%$ \\
\hline 13075 & $\mathrm{GA}$ & Cook & rural & 312 & $28.5 \%$ & 227.16 & 1.375 & $66.5 \%$ \\
\hline 13077 & GA & Coweta & urban & 1,958 & $28.6 \%$ & 440.89 & 4.440 & $54.9 \%$ \\
\hline 13081 & GA & Crisp & rural & 372 & $28.2 \%$ & 272.58 & 1.363 & $59.9 \%$ \\
\hline 13083 & GA & Dade & urban & 381 & $28.8 \%$ & 173.98 & 2.191 & $61.6 \%$ \\
\hline 13085 & GA & Dawson & urban & 536 & $28.7 \%$ & 210.83 & 2.544 & $61.6 \%$ \\
\hline 13089 & GA & DeKalb & urban & 7,249 & $28.4 \%$ & 267.58 & 27.091 & $47.3 \%$ \\
\hline 13087 & GA & Decatur & rural & 518 & $28.3 \%$ & 597.14 & 0.867 & $67.1 \%$ \\
\hline 13091 & GA & Dodge & rural & 460 & $28.6 \%$ & 495.89 & 0.928 & $64.9 \%$ \\
\hline 13093 & GA & Dooly & rural & 193 & $28.2 \%$ & 391.94 & 0.492 & $61.8 \%$ \\
\hline 13095 & $\mathrm{GA}$ & Dougherty & urban & 1,727 & $28.3 \%$ & 328.69 & 5.254 & $64.9 \%$ \\
\hline 13097 & $\mathrm{GA}$ & Douglas & urban & 1,480 & $28.8 \%$ & 200.07 & 7.396 & $52.3 \%$ \\
\hline 13099 & GA & Early & rural & 222 & $28.3 \%$ & 512.59 & 0.433 & $63.2 \%$ \\
\hline 13103 & GA & Effingham & urban & 701 & $28.6 \%$ & 477.70 & 1.467 & $62.8 \%$ \\
\hline 13105 & GA & Elbert & rural & 481 & $28.4 \%$ & 351.06 & 1.369 & $64.3 \%$ \\
\hline 13107 & GA & Emanuel & rural & 398 & $28.4 \%$ & 680.60 & 0.585 & $58.9 \%$ \\
\hline 13109 & GA & Evans & rural & 190 & $28.3 \%$ & 182.85 & 1.039 & $60.6 \%$ \\
\hline 13111 & $\mathrm{GA}$ & Fannin & rural & 833 & $28.7 \%$ & 386.72 & 2.154 & $70.7 \%$ \\
\hline 13113 & $\mathrm{GA}$ & Fayette & urban & 2,346 & $28.5 \%$ & 194.34 & 12.074 & $59.6 \%$ \\
\hline 13115 & GA & Floyd & urban & 1,944 & $28.5 \%$ & 509.91 & 3.812 & $67.1 \%$ \\
\hline 13117 & GA & Forsyth & urban & 2,189 & $28.9 \%$ & 224.02 & 9.772 & $55.7 \%$ \\
\hline 13119 & $\mathrm{GA}$ & Franklin & rural & 573 & $28.5 \%$ & 261.50 & 2.193 & $66.6 \%$ \\
\hline 13121 & $\mathrm{GA}$ & Fulton & urban & 10,608 & $28.3 \%$ & 526.63 & 20.143 & $52.1 \%$ \\
\hline 13123 & GA & Gilmer & rural & 830 & $28.6 \%$ & 426.54 & 1.945 & $67.6 \%$ \\
\hline 13127 & $\mathrm{GA}$ & Glynn & urban & 2,093 & $28.6 \%$ & 419.75 & 4.986 & $68.8 \%$ \\
\hline 13129 & GA & Gordon & rural & 1,011 & $28.6 \%$ & 355.81 & 2.842 & $68.6 \%$ \\
\hline 13131 & $\mathrm{GA}$ & Grady & rural & 418 & $28.3 \%$ & 454.53 & 0.920 & $62.3 \%$ \\
\hline 13133 & $\mathrm{GA}$ & Greene & rural & 621 & $28.4 \%$ & 387.44 & 1.603 & $68.9 \%$ \\
\hline 13135 & $\mathrm{GA}$ & Gwinnett & urban & 7,738 & $28.6 \%$ & 430.38 & 17.979 & $50.1 \%$ \\
\hline 13137 & GA & Habersham & rural & 996 & $28.6 \%$ & 276.74 & 3.600 & $62.0 \%$ \\
\hline 13139 & GA & Hall & urban & 3,286 & $28.6 \%$ & 392.78 & 8.366 & $61.1 \%$ \\
\hline 13141 & GA & Hancock & rural & 129 & $27.8 \%$ & 471.84 & 0.273 & $48.9 \%$ \\
\hline 13143 & GA & Haralson & urban & 527 & $28.5 \%$ & 282.17 & 1.868 & $59.7 \%$ \\
\hline 13145 & GA & Harris & urban & 483 & $28.4 \%$ & 463.87 & 1.041 & $60.3 \%$ \\
\hline 13147 & $\mathrm{GA}$ & Hart & rural & 533 & $28.3 \%$ & 232.39 & 2.295 & $69.6 \%$ \\
\hline 13149 & $\mathrm{GA}$ & Heard & urban & 140 & $28.7 \%$ & 296.03 & 0.474 & $56.7 \%$ \\
\hline 13151 & $\mathrm{GA}$ & Henry & urban & 2,326 & $28.6 \%$ & 322.13 & 7.222 & $50.7 \%$ \\
\hline 13153 & GA & Houston & urban & 2,212 & $28.6 \%$ & 375.54 & 5.891 & $73.4 \%$ \\
\hline 13155 & GA & Irwin & rural & 141 & $28.5 \%$ & 354.34 & 0.398 & $66.5 \%$ \\
\hline 13157 & GA & Jackson & rural & 1,580 & $28.8 \%$ & 339.66 & 4.652 & $61.2 \%$ \\
\hline 13159 & GA & Jasper & urban & 195 & $28.4 \%$ & 368.16 & 0.531 & $55.6 \%$ \\
\hline 13161 & GA & Jeff Davis & rural & 253 & $28.7 \%$ & 330.74 & 0.765 & $69.6 \%$ \\
\hline 13163 & GA & Jefferson & rural & 290 & $28.1 \%$ & 526.48 & 0.551 & $60.2 \%$ \\
\hline 13165 & GA & Jenkins & rural & 135 & $28.1 \%$ & 347.28 & 0.389 & $56.5 \%$ \\
\hline 13167 & $\mathrm{GA}$ & Johnson & rural & 138 & $28.4 \%$ & 303.01 & 0.456 & $60.5 \%$ \\
\hline 13169 & $\mathrm{GA}$ & Jones & urban & 253 & $28.2 \%$ & 393.93 & 0.643 & $53.4 \%$ \\
\hline 13171 & $\mathrm{GA}$ & Lamar & urban & 301 & $28.5 \%$ & 183.50 & 1.639 & $54.7 \%$ \\
\hline 13173 & GA & Lanier & urban & 104 & $28.4 \%$ & 185.26 & 0.560 & $66.7 \%$ \\
\hline 13175 & GA & Laurens & rural & 872 & $28.4 \%$ & 807.30 & 1.080 & $61.0 \%$ \\
\hline 13177 & GA & Lee & urban & 388 & $28.7 \%$ & 355.78 & 1.091 & $65.7 \%$ \\
\hline 13179 & GA & Liberty & urban & 560 & $28.8 \%$ & 489.80 & 1.144 & $68.0 \%$ \\
\hline 13181 & GA & Lincoln & urban & 174 & $28.0 \%$ & 210.38 & 0.829 & $62.5 \%$ \\
\hline 13185 & $\mathrm{GA}$ & Lowndes & urban & 1,670 & $28.6 \%$ & 496.07 & 3.366 & $67.9 \%$ \\
\hline 13187 & GA & Lumpkin & rural & 564 & $28.7 \%$ & 282.93 & 1.993 & $65.6 \%$ \\
\hline 13193 & GA & Macon & rural & 163 & $28.1 \%$ & 400.64 & 0.407 & $54.1 \%$ \\
\hline 13195 & $\mathrm{GA}$ & Madison & urban & 535 & $28.6 \%$ & 282.31 & 1.896 & $60.7 \%$ \\
\hline 13189 & GA & McDuffie & urban & 335 & $28.2 \%$ & 257.46 & 1.303 & $51.4 \%$ \\
\hline 13191 & $\mathrm{GA}$ & McIntosh & urban & 262 & $28.8 \%$ & 424.30 & 0.617 & $61.1 \%$ \\
\hline 13199 & GA & Meriwether & urban & 321 & $28.0 \%$ & 501.22 & 0.641 & $51.4 \%$ \\
\hline 13201 & GA & Miller & rural & 139 & $28.5 \%$ & 282.42 & 0.493 & $68.7 \%$ \\
\hline 13205 & GA & Mitchell & rural & 373 & $28.4 \%$ & 512.08 & 0.729 & $61.2 \%$ \\
\hline 13207 & GA & Monroe & urban & 381 & $28.4 \%$ & 395.66 & 0.962 & $62.5 \%$ \\
\hline 13209 & GA & Montgomery & rural & 147 & $28.4 \%$ & 239.52 & 0.614 & $61.1 \%$ \\
\hline 13211 & $\mathrm{GA}$ & Morgan & urban & 385 & $28.7 \%$ & 347.35 & 1.108 & $58.3 \%$ \\
\hline 13213 & $\mathrm{GA}$ & Murray & urban & 655 & $28.7 \%$ & 344.47 & 1.903 & $73.1 \%$ \\
\hline 13215 & GA & Muscogee & urban & 2,988 & $28.3 \%$ & 216.39 & 13.809 & $62.6 \%$ \\
\hline 13217 & GA & Newton & urban & 1,352 & $28.5 \%$ & 272.16 & 4.969 & $51.6 \%$ \\
\hline 13219 & GA & Oconee & urban & 654 & $28.6 \%$ & 184.29 & 3.550 & $62.9 \%$ \\
\hline 13221 & GA & Oglethorpe & urban & 178 & $28.3 \%$ & 439.01 & 0.406 & $64.9 \%$ \\
\hline
\end{tabular}




\begin{tabular}{|c|c|c|c|c|c|c|c|c|}
\hline 13223 & GA & $\begin{array}{l}\text { Paulding } \\
\end{array}$ & urban & 1,163 & $28.4 \%$ & 312.22 & 3.724 & $54.6 \%$ \\
\hline 13225 & GA & Peach & urban & 569 & $28.6 \%$ & 150.27 & 3.788 & $63.0 \%$ \\
\hline 13227 & GA & Pickens & urban & 956 & $28.7 \%$ & 232.06 & 4.121 & $63.9 \%$ \\
\hline 13229 & GA & Pierce & rural & 400 & $28.5 \%$ & 316.49 & 1.265 & $68.1 \%$ \\
\hline 13231 & GA & Pike & urban & 300 & $28.4 \%$ & 216.09 & 1.388 & $55.6 \%$ \\
\hline 13233 & GA & Polk & rural & 828 & $28.4 \%$ & 310.33 & 2.668 & $63.5 \%$ \\
\hline 13235 & GA & Pulaski & urban & 227 & $28.4 \%$ & 249.03 & 0.910 & $71.3 \%$ \\
\hline 13237 & GA & Putnam & rural & 531 & $28.4 \%$ & 344.64 & 1.539 & $64.4 \%$ \\
\hline 13241 & GA & Rabun & rural & 589 & $28.7 \%$ & 369.99 & 1.591 & $72.1 \%$ \\
\hline 13243 & GA & Randolph & rural & 140 & $28.1 \%$ & 428.24 & 0.326 & $59.7 \%$ \\
\hline 13247 & GA & Rockdale & urban & 1,054 & $28.7 \%$ & 129.79 & 8.124 & $48.9 \%$ \\
\hline 13251 & GA & Screven & rural & 248 & $28.1 \%$ & 645.10 & 0.385 & $59.3 \%$ \\
\hline 13253 & GA & Seminole & rural & 204 & $28.5 \%$ & 235.23 & 0.868 & $64.2 \%$ \\
\hline 13255 & GA & Spalding & urban & 1,159 & $28.5 \%$ & 196.47 & 5.901 & $56.5 \%$ \\
\hline 13257 & GA & Stephens & rural & 719 & $28.6 \%$ & 179.13 & 4.013 & $65.6 \%$ \\
\hline 13261 & GA & Sumter & rural & 526 & $28.4 \%$ & 482.70 & 1.089 & $58.7 \%$ \\
\hline 13263 & GA & Talbot & rural & 111 & $28.4 \%$ & 391.39 & 0.284 & $54.2 \%$ \\
\hline 13267 & GA & Tattnall & rural & 326 & $28.3 \%$ & 479.40 & 0.681 & $59.3 \%$ \\
\hline 13269 & GA & Taylor & rural & 147 & $28.4 \%$ & 376.69 & 0.391 & $57.1 \%$ \\
\hline 13273 & GA & Terrell & urban & 184 & $28.3 \%$ & 335.44 & 0.549 & $57.9 \%$ \\
\hline 13275 & GA & Thomas & rural & 1,075 & $28.3 \%$ & 544.60 & 1.974 & $64.0 \%$ \\
\hline 13277 & GA & Tift & rural & 739 & $28.4 \%$ & 258.91 & 2.855 & $63.7 \%$ \\
\hline 13279 & GA & Toombs & rural & 561 & $28.4 \%$ & 364.00 & 1.542 & $67.0 \%$ \\
\hline 13281 & GA & Towns & rural & 539 & $28.8 \%$ & 166.56 & 3.236 & $67.1 \%$ \\
\hline 13285 & GA & Troup & rural & 1,117 & $28.2 \%$ & 413.99 & 2.697 & $60.5 \%$ \\
\hline 13287 & GA & Turner & rural & 168 & $28.7 \%$ & 285.39 & 0.589 & $57.7 \%$ \\
\hline 13289 & GA & Twiggs & urban & 122 & $28.2 \%$ & 358.40 & 0.339 & $55.1 \%$ \\
\hline 13291 & GA & Union & rural & 899 & $28.7 \%$ & 321.93 & 2.794 & $68.2 \%$ \\
\hline 13293 & $\mathrm{GA}$ & Upson & rural & 491 & $28.2 \%$ & 323.44 & 1.518 & $55.5 \%$ \\
\hline 13295 & $\mathrm{GA}$ & Walker & urban & 1,453 & $28.7 \%$ & 446.38 & 3.254 & $59.7 \%$ \\
\hline 13297 & GA & Walton & urban & 1,663 & $28.5 \%$ & 325.68 & 5.105 & $54.7 \%$ \\
\hline 13299 & GA & Ware & rural & 763 & $28.3 \%$ & 892.46 & 0.855 & $67.3 \%$ \\
\hline 13301 & $\mathrm{GA}$ & Warren & rural & 104 & $28.5 \%$ & 284.30 & 0.364 & $57.3 \%$ \\
\hline 13303 & $\mathrm{GA}$ & Washington & rural & 300 & $28.0 \%$ & 678.45 & 0.443 & $54.3 \%$ \\
\hline 13305 & GA & Wayne & rural & 516 & $28.8 \%$ & 641.78 & 0.805 & $63.8 \%$ \\
\hline 13311 & $\mathrm{GA}$ & White & rural & 675 & $28.9 \%$ & 240.69 & 2.803 & $62.1 \%$ \\
\hline 13313 & GA & Whitfield & urban & 1,827 & $28.6 \%$ & 290.46 & 6.289 & $72.4 \%$ \\
\hline 13315 & $\mathrm{GA}$ & Wilcox & rural & 159 & $28.4 \%$ & 377.70 & 0.421 & $60.6 \%$ \\
\hline 15001 & $\mathrm{HI}$ & Hawaii & rural & 3,392 & $28.4 \%$ & $4,028.42$ & 0.842 & $46.3 \%$ \\
\hline 15003 & $\mathrm{HI}$ & Honolulu & urban & 11,678 & $28.2 \%$ & 600.74 & 19.439 & $34.8 \%$ \\
\hline 15007 & $\mathrm{HI}$ & Kauai & rural & 1,298 & $28.3 \%$ & 619.96 & 2.093 & $47.9 \%$ \\
\hline 15009 & $\mathrm{HI}$ & Maui & urban & 1,960 & $28.4 \%$ & $1,161.52$ & 1.687 & $36.1 \%$ \\
\hline 19001 & IA & Adair & rural & 236 & $29.0 \%$ & 569.27 & 0.414 & $85.0 \%$ \\
\hline 19003 & $\mathrm{IA}$ & Adams & rural & 140 & $28.5 \%$ & 423.44 & 0.331 & $89.6 \%$ \\
\hline 19005 & IA & Allamakee & rural & 538 & $28.4 \%$ & 639.08 & 0.842 & $83.2 \%$ \\
\hline 19007 & IA & Appanoose & rural & 430 & $28.5 \%$ & 497.29 & 0.864 & $79.3 \%$ \\
\hline 19009 & IA & Audubon & rural & 251 & $28.5 \%$ & 442.96 & 0.566 & $90.5 \%$ \\
\hline 19011 & IA & Benton & urban & 638 & $28.6 \%$ & 716.26 & 0.891 & $72.5 \%$ \\
\hline 19013 & IA & Black Hawk & urban & 3,217 & $28.4 \%$ & 565.77 & 5.687 & $77.4 \%$ \\
\hline 19015 & $\mathrm{IA}$ & Boone & rural & 711 & $28.5 \%$ & 571.57 & 1.244 & $79.3 \%$ \\
\hline 19017 & $\mathrm{IA}$ & Bremer & urban & 873 & $28.7 \%$ & 435.48 & 2.004 & $85.3 \%$ \\
\hline 19019 & IA & Buchanan & rural & 597 & $28.6 \%$ & 571.02 & 1.046 & $79.4 \%$ \\
\hline 19021 & $\mathrm{IA}$ & Buena Vista & rural & 540 & $28.5 \%$ & 574.91 & 0.939 & $87.3 \%$ \\
\hline 19023 & $\mathrm{IA}$ & Butler & rural & 573 & $28.4 \%$ & 580.13 & 0.988 & $84.1 \%$ \\
\hline 19025 & IA & Calhoun & rural & 401 & $28.6 \%$ & 569.97 & 0.704 & $90.3 \%$ \\
\hline 19027 & IA & Carroll & rural & 760 & $28.5 \%$ & 569.44 & 1.334 & $85.7 \%$ \\
\hline 19029 & IA & Cass & rural & 522 & $28.7 \%$ & 564.27 & 0.924 & $84.5 \%$ \\
\hline 19031 & $\mathrm{IA}$ & Cedar & rural & 478 & $28.7 \%$ & 579.44 & 0.824 & $72.2 \%$ \\
\hline 19033 & $\mathrm{IA}$ & Cerro Gordo & rural & 1,630 & $28.5 \%$ & 568.31 & 2.868 & $89.9 \%$ \\
\hline 19035 & IA & Cherokee & rural & 434 & $28.5 \%$ & 576.91 & 0.752 & $83.0 \%$ \\
\hline 19037 & IA & Chickasaw & rural & 422 & $28.6 \%$ & 504.38 & 0.837 & $91.3 \%$ \\
\hline 19039 & $\mathrm{IA}$ & Clarke & rural & 272 & $28.7 \%$ & 431.17 & 0.631 & $82.4 \%$ \\
\hline 19041 & IA & Clay & rural & 651 & $28.4 \%$ & 567.24 & 1.147 & $94.0 \%$ \\
\hline 19043 & IA & Clayton & rural & 622 & $28.4 \%$ & 778.54 & 0.799 & $75.9 \%$ \\
\hline 19045 & IA & Clinton & rural & 1,319 & $28.4 \%$ & 694.91 & 1.898 & $76.5 \%$ \\
\hline 19047 & IA & Crawford & rural & 506 & $28.5 \%$ & 714.19 & 0.708 & $85.3 \%$ \\
\hline 19049 & IA & Dallas & urban & 1,024 & $28.6 \%$ & 588.45 & 1.741 & $72.6 \%$ \\
\hline 19051 & IA & Davis & rural & 257 & $28.7 \%$ & 502.19 & 0.511 & $85.5 \%$ \\
\hline 19053 & IA & Decatur & rural & 264 & $28.7 \%$ & 531.88 & 0.496 & $83.0 \%$ \\
\hline 19055 & IA & Delaware & rural & 436 & $28.6 \%$ & 577.76 & 0.755 & $74.9 \%$ \\
\hline 19057 & $\mathrm{IA}$ & Des Moines & rural & 1,433 & $28.5 \%$ & 416.12 & 3.445 & $86.4 \%$ \\
\hline 19059 & IA & Dickinson & rural & 805 & $28.7 \%$ & 380.61 & 2.115 & $90.4 \%$ \\
\hline 19061 & IA & Dubuque & urban & 1,725 & $28.5 \%$ & 608.30 & 2.836 & $47.7 \%$ \\
\hline 19063 & IA & Emmet & rural & 361 & $28.5 \%$ & 395.88 & 0.913 & $91.0 \%$ \\
\hline 19065 & IA & Fayette & rural & 678 & $28.4 \%$ & 730.81 & 0.928 & $83.6 \%$ \\
\hline
\end{tabular}




\begin{tabular}{|c|c|c|c|c|c|c|c|c|}
\hline 19071 & $\mathrm{IA}$ & Fremont & rural & 237 & $28.4 \%$ & 511.15 & 0.463 & $84.3 \%$ \\
\hline 19073 & IA & Greene & rural & 324 & $28.4 \%$ & 569.57 & 0.568 & $80.5 \%$ \\
\hline 19075 & IA & Grundy & urban & 399 & $28.5 \%$ & 501.86 & 0.795 & $83.6 \%$ \\
\hline 19077 & IA & Guthrie & urban & 401 & $28.7 \%$ & 590.62 & 0.680 & $76.6 \%$ \\
\hline 19079 & IA & Hamilton & rural & 491 & $28.4 \%$ & 576.75 & 0.851 & $83.4 \%$ \\
\hline 19081 & IA & Hancock & rural & 394 & $28.4 \%$ & 571.00 & 0.690 & $91.6 \%$ \\
\hline 19083 & IA & Hardin & rural & 682 & $28.6 \%$ & 569.31 & 1.199 & $86.8 \%$ \\
\hline 19085 & IA & Harrison & urban & 478 & $28.5 \%$ & 696.85 & 0.687 & $83.9 \%$ \\
\hline 19087 & IA & Henry & rural & 657 & $28.6 \%$ & 434.33 & 1.513 & $84.5 \%$ \\
\hline 19089 & IA & Howard & rural & 381 & $28.4 \%$ & 473.25 & 0.805 & $91.9 \%$ \\
\hline 19093 & IA & Ida & rural & 267 & $28.5 \%$ & 431.51 & 0.620 & $83.9 \%$ \\
\hline 19095 & $\mathrm{IA}$ & lowa & rural & 443 & $28.3 \%$ & 586.46 & 0.756 & $74.1 \%$ \\
\hline 19097 & $\mathrm{IA}$ & Jackson & rural & 550 & $28.6 \%$ & 636.04 & 0.865 & $67.8 \%$ \\
\hline 19099 & IA & Jasper & rural & 1,037 & $28.6 \%$ & 730.42 & 1.420 & $80.9 \%$ \\
\hline 19101 & IA & Jefferson & rural & 579 & $28.5 \%$ & 435.51 & 1.330 & $80.6 \%$ \\
\hline 19103 & IA & Johnson & urban & 2,286 & $28.5 \%$ & 614.04 & 3.722 & $70.7 \%$ \\
\hline 19105 & IA & Jones & urban & 533 & $28.4 \%$ & 575.62 & 0.926 & $71.0 \%$ \\
\hline 19107 & IA & Keokuk & rural & 362 & $28.6 \%$ & 579.18 & 0.626 & $75.3 \%$ \\
\hline 19109 & $\mathrm{IA}$ & Kossuth & rural & 609 & $28.5 \%$ & 972.72 & 0.626 & $92.4 \%$ \\
\hline 19115 & IA & Louisa & rural & 293 & $28.5 \%$ & 401.77 & 0.729 & $77.2 \%$ \\
\hline 19117 & IA & Lucas & rural & 278 & $28.4 \%$ & 430.59 & 0.645 & $77.7 \%$ \\
\hline 19119 & $\mathrm{IA}$ & Lyon & rural & 376 & $28.2 \%$ & 587.65 & 0.639 & $87.2 \%$ \\
\hline 19121 & IA & Madison & urban & 401 & $28.5 \%$ & 561.01 & 0.715 & $74.4 \%$ \\
\hline 19123 & IA & Mahaska & rural & 620 & $28.4 \%$ & 570.86 & 1.087 & $80.3 \%$ \\
\hline 19125 & IA & Marion & rural & 1,010 & $28.5 \%$ & 554.53 & 1.821 & $83.8 \%$ \\
\hline 19127 & IA & Marshall & rural & 1,202 & $28.6 \%$ & 572.50 & 2.100 & $79.7 \%$ \\
\hline 19129 & $\mathrm{IA}$ & Mills & urban & 369 & $28.2 \%$ & 437.44 & 0.845 & $77.5 \%$ \\
\hline 19131 & IA & Mitchell & rural & 485 & $28.4 \%$ & 469.13 & 1.034 & $94.2 \%$ \\
\hline 19133 & $\mathrm{IA}$ & Monona & rural & 311 & $28.4 \%$ & 694.07 & 0.448 & $76.2 \%$ \\
\hline 19135 & IA & Monroe & rural & 249 & $28.4 \%$ & 433.72 & 0.574 & $83.1 \%$ \\
\hline 19137 & IA & Montgomery & rural & 366 & $28.3 \%$ & 424.10 & 0.863 & $86.6 \%$ \\
\hline 19139 & IA & Muscatine & rural & 958 & $28.5 \%$ & 437.47 & 2.191 & $67.8 \%$ \\
\hline 19141 & IA & O'Brien & rural & 570 & $28.3 \%$ & 573.04 & 0.995 & $90.4 \%$ \\
\hline 19143 & IA & Osceola & rural & 216 & $28.5 \%$ & 398.68 & 0.541 & $87.6 \%$ \\
\hline 19145 & IA & Page & rural & 514 & $28.3 \%$ & 534.94 & 0.962 & $86.8 \%$ \\
\hline 19147 & IA & Palo Alto & rural & 375 & $28.4 \%$ & 563.84 & 0.664 & $92.0 \%$ \\
\hline 19149 & $\mathrm{IA}$ & Plymouth & urban & 658 & $28.4 \%$ & 862.89 & 0.762 & $77.4 \%$ \\
\hline 19157 & IA & Poweshiek & rural & 627 & $28.7 \%$ & 584.93 & 1.072 & $80.7 \%$ \\
\hline 19159 & IA & Ringgold & rural & 193 & $28.5 \%$ & 535.50 & 0.360 & $87.3 \%$ \\
\hline 19161 & IA & Sac & rural & 424 & $28.3 \%$ & 575.01 & 0.738 & $90.3 \%$ \\
\hline 19163 & $\mathrm{IA}$ & Scott & urban & 3,754 & $28.5 \%$ & 458.09 & 8.194 & $67.2 \%$ \\
\hline 19165 & $\mathrm{IA}$ & Shelby & rural & 426 & $28.5 \%$ & 590.78 & 0.722 & $86.3 \%$ \\
\hline 19167 & $\mathrm{IA}$ & Sioux & rural & 971 & $28.6 \%$ & 768.33 & 1.263 & $87.7 \%$ \\
\hline 19169 & IA & Story & urban & 1,849 & $28.6 \%$ & 572.82 & 3.227 & $76.4 \%$ \\
\hline 19171 & IA & Tama & rural & 553 & $28.5 \%$ & 721.01 & 0.767 & $79.6 \%$ \\
\hline 19173 & IA & Taylor & rural & 255 & $28.5 \%$ & 531.90 & 0.480 & $93.7 \%$ \\
\hline 19175 & $\mathrm{IA}$ & Union & rural & 488 & $28.6 \%$ & 423.65 & 1.152 & $84.7 \%$ \\
\hline 19177 & $\mathrm{IA}$ & Van Buren & rural & 289 & $28.5 \%$ & 484.79 & 0.596 & $81.6 \%$ \\
\hline 19179 & $\mathrm{IA}$ & Wapello & rural & 1,045 & $28.6 \%$ & 431.83 & 2.420 & $83.3 \%$ \\
\hline 19181 & $\mathrm{IA}$ & Warren & urban & 1,130 & $28.5 \%$ & 569.83 & 1.983 & $72.8 \%$ \\
\hline 19183 & IA & Washington & urban & 704 & $28.5 \%$ & 568.84 & 1.237 & $78.3 \%$ \\
\hline 19185 & $\mathrm{IA}$ & Wayne & rural & 215 & $28.8 \%$ & 525.44 & 0.409 & $79.5 \%$ \\
\hline 19187 & IA & Webster & rural & 1,195 & $28.5 \%$ & 715.62 & 1.669 & $84.3 \%$ \\
\hline 19189 & IA & Winnebago & rural & 428 & $28.6 \%$ & 400.49 & 1.070 & $88.4 \%$ \\
\hline 19191 & $\mathrm{IA}$ & Winneshiek & rural & 677 & $28.4 \%$ & 689.87 & 0.982 & $77.6 \%$ \\
\hline 19193 & IA & Woodbury & urban & 2,087 & $28.5 \%$ & 872.83 & 2.391 & $65.9 \%$ \\
\hline 19195 & IA & Worth & rural & 256 & $28.5 \%$ & 400.12 & 0.639 & $85.8 \%$ \\
\hline 19197 & IA & Wright & rural & 506 & $28.4 \%$ & 580.42 & 0.871 & $90.9 \%$ \\
\hline 16001 & ID & Ada & urban & 5,658 & $28.5 \%$ & $1,052.58$ & 5.375 & $41.5 \%$ \\
\hline 16003 & ID & Adams & rural & 166 & $28.4 \%$ & $1,363.06$ & 0.122 & $74.1 \%$ \\
\hline 16005 & ID & Bannock & urban & 1,332 & $28.5 \%$ & $1,111.99$ & 1.198 & $56.3 \%$ \\
\hline 16007 & ID & Bear Lake & rural & 216 & $28.4 \%$ & 974.78 & 0.222 & $89.4 \%$ \\
\hline 16009 & ID & Benewah & rural & 313 & $28.6 \%$ & 776.62 & 0.403 & $68.0 \%$ \\
\hline 16011 & ID & Bingham & rural & 711 & $28.5 \%$ & $2,093.98$ & 0.340 & $64.8 \%$ \\
\hline 16013 & ID & Blaine & rural & 650 & $28.8 \%$ & $2,643.59$ & 0.246 & $69.0 \%$ \\
\hline 16015 & ID & Boise & urban & 118 & $27.8 \%$ & $1,899.24$ & 0.062 & $42.8 \%$ \\
\hline 16017 & ID & Bonner & rural & 1,236 & $28.5 \%$ & $1,734.57$ & 0.712 & $60.9 \%$ \\
\hline 16019 & ID & Bonneville & urban & 1,853 & $28.7 \%$ & $1,866.08$ & 0.993 & $66.6 \%$ \\
\hline 16021 & ID & Boundary & rural & 331 & $28.4 \%$ & $1,268.56$ & 0.261 & $59.4 \%$ \\
\hline 16027 & ID & Canyon & urban & 2,287 & $28.5 \%$ & 587.37 & 3.893 & $37.5 \%$ \\
\hline 16029 & ID & Caribou & rural & 189 & $28.8 \%$ & $1,764.15$ & 0.107 & $76.6 \%$ \\
\hline 16031 & ID & Cassia & rural & 446 & $28.5 \%$ & $2,565.08$ & 0.174 & $68.6 \%$ \\
\hline 16035 & ID & Clearwater & rural & 371 & $28.3 \%$ & $2,457.27$ & 0.151 & $88.6 \%$ \\
\hline 16037 & ID & Custer & rural & 188 & $28.4 \%$ & $4,920.94$ & 0.038 & $87.9 \%$ \\
\hline
\end{tabular}




\begin{tabular}{|c|c|c|c|c|c|c|c|c|}
\hline 16043 & ID & Fremont & rural & 290 & $28.5 \%$ & $1,863.53$ & 0.156 & $73.6 \%$ \\
\hline 16045 & ID & Gem & urban & 348 & $28.5 \%$ & 560.90 & 0.620 & $43.5 \%$ \\
\hline 16047 & ID & Gooding & rural & 346 & $28.9 \%$ & 728.97 & 0.475 & $66.8 \%$ \\
\hline 16049 & ID & Idaho & rural & 666 & $28.5 \%$ & $8,477.35$ & 0.079 & $90.5 \%$ \\
\hline 16051 & ID & Jefferson & urban & 405 & $28.4 \%$ & $1,093.50$ & 0.371 & $65.9 \%$ \\
\hline 16053 & ID & Jerome & rural & 361 & $28.5 \%$ & 597.18 & 0.604 & $57.6 \%$ \\
\hline 16055 & ID & Kootenai & urban & 3,665 & $28.7 \%$ & $1,244.12$ & 2.946 & $58.5 \%$ \\
\hline 16057 & ID & Latah & rural & 796 & $28.6 \%$ & $1,076.00$ & 0.740 & $73.2 \%$ \\
\hline 16059 & ID & Lemhi & rural & 414 & $28.4 \%$ & $4,563.39$ & 0.091 & $91.5 \%$ \\
\hline 16061 & ID & Lewis & rural & 275 & $28.5 \%$ & 478.80 & 0.574 & $86.7 \%$ \\
\hline 16067 & ID & Minidoka & rural & 440 & $28.4 \%$ & 757.59 & 0.580 & $65.5 \%$ \\
\hline 16069 & ID & Nez Perce & urban & 1,100 & $28.7 \%$ & 848.09 & 1.296 & $67.1 \%$ \\
\hline 16071 & ID & Oneida & rural & 120 & $28.7 \%$ & $1,200.06$ & 0.100 & $72.7 \%$ \\
\hline 16073 & ID & Owyhee & urban & 160 & $28.3 \%$ & $7,665.51$ & 0.021 & $46.2 \%$ \\
\hline 16075 & ID & Payette & rural & 443 & $28.6 \%$ & 406.87 & 1.089 & $51.3 \%$ \\
\hline 16077 & ID & Power & rural & 140 & $28.6 \%$ & $1,404.24$ & 0.100 & $69.7 \%$ \\
\hline 16079 & ID & Shoshone & rural & 379 & $28.5 \%$ & $2,629.66$ & 0.144 & $70.0 \%$ \\
\hline 16081 & ID & Teton & rural & 194 & $28.3 \%$ & 449.46 & 0.432 & $86.7 \%$ \\
\hline 16083 & ID & Twin Falls & rural & 1,673 & $28.6 \%$ & 1921.21 & 0.871 & $60.8 \%$ \\
\hline 17001 & $\mathrm{IL}$ & Adams & rural & 1,938 & $28.6 \%$ & 855.20 & 2.266 & $78.7 \%$ \\
\hline 17003 & $\mathrm{IL}$ & Alexander & urban & 191 & $28.2 \%$ & 235.51 & 0.812 & $83.6 \%$ \\
\hline 17005 & $\mathrm{IL}$ & Bond & urban & 428 & $28.6 \%$ & 380.28 & 1.124 & $78.9 \%$ \\
\hline 17007 & $\mathrm{IL}$ & Boone & urban & 920 & $28.6 \%$ & 280.72 & 3.277 & $62.8 \%$ \\
\hline 17009 & $\mathrm{IL}$ & Brown & rural & 134 & $28.8 \%$ & 305.61 & 0.439 & $78.7 \%$ \\
\hline 17011 & $\mathrm{IL}$ & Bureau & rural & 1,103 & $28.6 \%$ & 869.03 & 1.269 & $80.3 \%$ \\
\hline 17013 & $\mathrm{IL}$ & Calhoun & urban & 154 & $28.6 \%$ & 253.82 & 0.606 & $78.8 \%$ \\
\hline 17015 & $\mathrm{IL}$ & Carroll & rural & 510 & $28.6 \%$ & 444.81 & 1.146 & $70.7 \%$ \\
\hline 17017 & $\mathrm{IL}$ & Cass & rural & 317 & $28.5 \%$ & 375.82 & 0.843 & $75.2 \%$ \\
\hline 17019 & $\mathrm{IL}$ & Champaign & urban & 2,077 & $28.4 \%$ & 996.27 & 2.084 & $42.3 \%$ \\
\hline 17021 & $\mathrm{IL}$ & Christian & rural & 938 & $28.6 \%$ & 709.38 & 1.322 & $76.9 \%$ \\
\hline 17023 & $\mathrm{IL}$ & Clark & rural & 467 & $28.8 \%$ & 501.42 & 0.931 & $73.2 \%$ \\
\hline 17025 & $\mathrm{IL}$ & Clay & rural & 427 & $28.6 \%$ & 468.32 & 0.912 & $85.9 \%$ \\
\hline 17027 & $\mathrm{IL}$ & Clinton & urban & 872 & $28.6 \%$ & 474.09 & 1.840 & $81.8 \%$ \\
\hline 17029 & $\mathrm{IL}$ & Coles & rural & 993 & $28.5 \%$ & 508.29 & 1.953 & $66.0 \%$ \\
\hline 17031 & $\mathrm{IL}$ & Cook & urban & 81,910 & $28.4 \%$ & 945.33 & 86.647 & $65.0 \%$ \\
\hline 17033 & $\mathrm{IL}$ & Crawford & rural & 593 & $28.5 \%$ & 443.63 & 1.337 & $86.4 \%$ \\
\hline 17035 & $\mathrm{IL}$ & Cumberland & rural & 260 & $28.6 \%$ & 346.02 & 0.751 & $72.1 \%$ \\
\hline 17043 & $\mathrm{IL}$ & DuPage & urban & 19,811 & $28.6 \%$ & 327.50 & 60.493 & $69.8 \%$ \\
\hline 17045 & $\mathrm{IL}$ & Edgar & rural & 507 & $28.5 \%$ & 623.37 & 0.813 & $75.6 \%$ \\
\hline 17047 & $\mathrm{IL}$ & Edwards & rural & 195 & $28.5 \%$ & 222.42 & 0.878 & $85.9 \%$ \\
\hline 17049 & $\mathrm{IL}$ & Effingham & rural & 1,067 & $28.6 \%$ & 478.78 & 2.230 & $83.5 \%$ \\
\hline 17051 & $\mathrm{IL}$ & Fayette & rural & 508 & $28.8 \%$ & 716.48 & 0.709 & $81.4 \%$ \\
\hline 17053 & $\mathrm{IL}$ & Ford & urban & 327 & $28.6 \%$ & 485.62 & 0.673 & $69.0 \%$ \\
\hline 17055 & $\mathrm{IL}$ & Franklin & rural & 1,092 & $28.7 \%$ & 408.89 & 2.670 & $71.5 \%$ \\
\hline 17057 & $\mathrm{IL}$ & Fulton & rural & 950 & $28.4 \%$ & 865.59 & 1.097 & $66.3 \%$ \\
\hline 17059 & $\mathrm{IL}$ & Gallatin & rural & 189 & $28.5 \%$ & 323.07 & 0.586 & $81.8 \%$ \\
\hline 17061 & $\mathrm{IL}$ & Greene & rural & 389 & $28.6 \%$ & 543.02 & 0.716 & $81.8 \%$ \\
\hline 17063 & $\mathrm{IL}$ & Grundy & urban & 1,118 & $28.6 \%$ & 418.04 & 2.674 & $82.1 \%$ \\
\hline 17065 & $\mathrm{IL}$ & Hamilton & rural & 252 & $28.5 \%$ & 434.67 & 0.581 & $80.9 \%$ \\
\hline 17067 & $\mathrm{IL}$ & Hancock & rural & 675 & $28.7 \%$ & 793.73 & 0.851 & $81.8 \%$ \\
\hline 17069 & $\mathrm{IL}$ & Hardin & rural & 123 & $28.3 \%$ & 177.53 & 0.692 & $78.3 \%$ \\
\hline 17071 & $\mathrm{IL}$ & Henderson & rural & 237 & $28.5 \%$ & 378.87 & 0.626 & $79.6 \%$ \\
\hline 17073 & $\mathrm{IL}$ & Henry & urban & 1,481 & $28.5 \%$ & 822.99 & 1.799 & $76.3 \%$ \\
\hline 17075 & $\mathrm{IL}$ & Iroquois & rural & 907 & $28.8 \%$ & $1,117.32$ & 0.812 & $77.1 \%$ \\
\hline 17077 & $\mathrm{IL}$ & Jackson & urban & 1,075 & $28.5 \%$ & 584.08 & 1.841 & $66.5 \%$ \\
\hline 17079 & $\mathrm{IL}$ & Jasper & rural & 264 & $28.5 \%$ & 494.51 & 0.534 & $83.1 \%$ \\
\hline 17081 & $\mathrm{IL}$ & Jefferson & rural & 1,003 & $28.5 \%$ & 571.17 & 1.756 & $79.1 \%$ \\
\hline 17083 & $\mathrm{IL}$ & Jersey & urban & 455 & $28.5 \%$ & 369.27 & 1.231 & $76.0 \%$ \\
\hline 17085 & $\mathrm{IL}$ & Jo Daviess & rural & 632 & $28.5 \%$ & 601.09 & 1.051 & $54.4 \%$ \\
\hline 17087 & $\mathrm{IL}$ & Johnson & rural & 354 & $28.9 \%$ & 343.92 & 1.029 & $69.5 \%$ \\
\hline 17089 & $\mathrm{IL}$ & Kane & urban & 9,257 & $28.7 \%$ & 520.06 & 17.799 & $67.2 \%$ \\
\hline 17091 & $\mathrm{IL}$ & Kankakee & urban & 2,449 & $28.5 \%$ & 676.56 & 3.620 & $77.0 \%$ \\
\hline 17093 & IL & Kendall & urban & 1,283 & $28.7 \%$ & 320.34 & 4.005 & $69.4 \%$ \\
\hline 17095 & $\mathrm{IL}$ & Knox & rural & 1,414 & $28.5 \%$ & 716.39 & 1.973 & $65.5 \%$ \\
\hline 17099 & $\mathrm{IL}$ & LaSalle & rural & 3,103 & $28.6 \%$ & $1,135.12$ & 2.734 & $80.0 \%$ \\
\hline 17097 & $\mathrm{IL}$ & Lake & urban & 13,989 & $28.7 \%$ & 443.67 & 31.530 & $76.8 \%$ \\
\hline 17101 & $\mathrm{IL}$ & Lawrence & rural & 432 & $28.8 \%$ & 372.18 & 1.162 & $86.3 \%$ \\
\hline 17103 & $\mathrm{IL}$ & Lee & rural & 887 & $28.5 \%$ & 724.90 & 1.224 & $72.4 \%$ \\
\hline 17105 & IL & Livingston & rural & 829 & $28.6 \%$ & $1,044.29$ & 0.794 & $70.0 \%$ \\
\hline 17107 & $\mathrm{IL}$ & Logan & rural & 654 & $28.5 \%$ & 618.06 & 1.058 & $68.2 \%$ \\
\hline 17115 & $\mathrm{IL}$ & Macon & urban & 3,016 & $28.4 \%$ & 580.69 & 5.193 & $79.5 \%$ \\
\hline 17117 & $\mathrm{IL}$ & Macoupin & urban & 1,401 & $28.6 \%$ & 862.91 & 1.624 & $80.2 \%$ \\
\hline 17119 & $\mathrm{IL}$ & Madison & urban & 4,961 & $28.5 \%$ & 715.58 & 6.933 & $57.7 \%$ \\
\hline 17121 & $\mathrm{IL}$ & Marion & rural & 1,248 & $28.4 \%$ & 572.36 & 2.181 & $82.1 \%$ \\
\hline
\end{tabular}




\begin{tabular}{|c|c|c|c|c|c|c|c|c|}
\hline 17127 & $\mathrm{IL}$ & Massac & rural & 390 & $28.7 \%$ & 237.22 & 1.643 & $77.8 \%$ \\
\hline 17109 & $\mathrm{IL}$ & McDonough & rural & 635 & $28.3 \%$ & 589.41 & 1.077 & $63.8 \%$ \\
\hline 17111 & $\mathrm{IL}$ & McHenry & urban & 7,514 & $28.8 \%$ & 603.17 & 12.458 & $78.5 \%$ \\
\hline 17113 & $\mathrm{IL}$ & McLean & urban & 2,828 & $28.5 \%$ & $1,183.38$ & 2.390 & $63.1 \%$ \\
\hline 17129 & IL & Menard & urban & 284 & $28.5 \%$ & 314.44 & 0.902 & $61.8 \%$ \\
\hline 17131 & $\mathrm{IL}$ & Mercer & urban & 450 & $28.6 \%$ & 561.20 & 0.801 & $70.4 \%$ \\
\hline 17133 & $\mathrm{IL}$ & Monroe & urban & 601 & $28.3 \%$ & 385.01 & 1.560 & $53.6 \%$ \\
\hline 17135 & $\mathrm{IL}$ & Montgomery & rural & 898 & $28.5 \%$ & 703.69 & 1.277 & $81.3 \%$ \\
\hline 17137 & $\mathrm{IL}$ & Morgan & rural & 886 & $28.5 \%$ & 568.79 & 1.558 & $72.2 \%$ \\
\hline 17139 & $\mathrm{IL}$ & Moultrie & rural & 447 & $28.3 \%$ & 335.94 & 1.330 & $77.7 \%$ \\
\hline 17143 & $\mathrm{IL}$ & Peoria & urban & 3,858 & $28.4 \%$ & 619.21 & 6.230 & $62.9 \%$ \\
\hline 17145 & $\mathrm{IL}$ & Perry & rural & 515 & $28.6 \%$ & 441.76 & 1.167 & $73.9 \%$ \\
\hline 17147 & $\mathrm{IL}$ & Piatt & urban & 364 & $28.3 \%$ & 439.20 & 0.828 & $52.1 \%$ \\
\hline 17149 & $\mathrm{IL}$ & Pike & rural & 484 & $28.7 \%$ & 831.38 & 0.582 & $82.5 \%$ \\
\hline 17151 & $\mathrm{IL}$ & Pope & rural & 124 & $28.5 \%$ & 368.77 & 0.335 & $75.9 \%$ \\
\hline 17153 & $\mathrm{IL}$ & Pulaski & rural & 159 & $28.5 \%$ & 199.18 & 0.797 & $77.7 \%$ \\
\hline 17155 & $\mathrm{IL}$ & Putnam & rural & 199 & $28.5 \%$ & 160.16 & 1.241 & $79.4 \%$ \\
\hline 17157 & $\mathrm{IL}$ & Randolph & rural & 833 & $28.6 \%$ & 575.50 & 1.447 & $74.0 \%$ \\
\hline 17159 & $\mathrm{IL}$ & Richland & rural & 516 & $28.6 \%$ & 359.99 & 1.434 & $83.1 \%$ \\
\hline 17167 & $\mathrm{IL}$ & Sangamon & urban & 3,650 & $28.5 \%$ & 868.30 & 4.204 & $55.4 \%$ \\
\hline 17169 & $\mathrm{IL}$ & Schuyler & rural & 213 & $28.3 \%$ & 437.27 & 0.487 & $78.0 \%$ \\
\hline 17171 & $\mathrm{IL}$ & Scott & rural & 138 & $28.6 \%$ & 250.91 & 0.548 & $76.8 \%$ \\
\hline 17173 & $\mathrm{IL}$ & Shelby & rural & 709 & $28.6 \%$ & 758.52 & 0.935 & $84.7 \%$ \\
\hline 17163 & $\mathrm{IL}$ & St. Clair & urban & 4,049 & $28.4 \%$ & 657.76 & 6.155 & $55.9 \%$ \\
\hline 17175 & $\mathrm{IL}$ & Stark & urban & 180 & $28.2 \%$ & 288.08 & 0.626 & $71.2 \%$ \\
\hline 17177 & $\mathrm{IL}$ & Stephenson & rural & 1,167 & $28.5 \%$ & 564.52 & 2.067 & $54.4 \%$ \\
\hline 17179 & $\mathrm{IL}$ & Tazewell & urban & 3,343 & $28.4 \%$ & 648.97 & 5.151 & $67.5 \%$ \\
\hline 17181 & $\mathrm{IL}$ & Union & rural & 439 & $28.7 \%$ & 413.46 & 1.061 & $68.5 \%$ \\
\hline 17183 & $\mathrm{IL}$ & Vermilion & urban & 1,471 & $28.4 \%$ & 898.37 & 1.637 & $50.9 \%$ \\
\hline 17185 & $\mathrm{IL}$ & Wabash & rural & 347 & $28.2 \%$ & 223.25 & 1.555 & $84.3 \%$ \\
\hline 17187 & $\mathrm{IL}$ & Warren & rural & 481 & $28.6 \%$ & 542.41 & 0.887 & $73.0 \%$ \\
\hline 17189 & $\mathrm{IL}$ & Washington & rural & 381 & $28.3 \%$ & 562.57 & 0.678 & $79.3 \%$ \\
\hline 17191 & $\mathrm{IL}$ & Wayne & rural & 530 & $28.8 \%$ & 713.81 & 0.742 & $85.7 \%$ \\
\hline 17193 & $\mathrm{IL}$ & White & rural & 505 & $28.7 \%$ & 494.77 & 1.020 & $80.8 \%$ \\
\hline 17195 & $\mathrm{IL}$ & Whiteside & rural & 1,695 & $28.5 \%$ & 684.25 & 2.477 & $75.7 \%$ \\
\hline 17197 & $\mathrm{IL}$ & Will & urban & 9,993 & $28.7 \%$ & 836.91 & 11.940 & $72.3 \%$ \\
\hline 17199 & $\mathrm{IL}$ & Williamson & urban & 1,573 & $28.6 \%$ & 420.15 & 3.743 & $72.5 \%$ \\
\hline 18003 & IN & Allen & urban & 4,698 & $28.5 \%$ & 657.31 & 7.147 & $44.1 \%$ \\
\hline 18005 & IN & Bartholomew & urban & 1,890 & $28.6 \%$ & 406.91 & 4.645 & $72.3 \%$ \\
\hline 18007 & IN & Benton & urban & 231 & $28.6 \%$ & 406.42 & 0.569 & $76.0 \%$ \\
\hline 18009 & IN & Blackford & rural & 358 & $28.8 \%$ & 165.08 & 2.171 & $71.7 \%$ \\
\hline 18011 & IN & Boone & urban & 1,058 & $28.5 \%$ & 422.91 & 2.501 & $64.7 \%$ \\
\hline 18013 & IN & Brown & urban & 266 & $28.5 \%$ & 311.98 & 0.853 & $66.8 \%$ \\
\hline 18015 & IN & Carroll & urban & 390 & $28.6 \%$ & 372.22 & 1.048 & $71.4 \%$ \\
\hline 18017 & IN & Cass & rural & 815 & $28.5 \%$ & 412.15 & 1.979 & $69.0 \%$ \\
\hline 18019 & IN & Clark & urban & 2,275 & $28.6 \%$ & 372.86 & 6.102 & $65.9 \%$ \\
\hline 18021 & IN & Clay & urban & 741 & $28.6 \%$ & 357.54 & 2.073 & $75.8 \%$ \\
\hline 18023 & IN & Clinton & rural & 721 & $28.5 \%$ & 405.07 & 1.779 & $72.2 \%$ \\
\hline 18025 & IN & Crawford & rural & 296 & $28.4 \%$ & 305.64 & 0.970 & $73.8 \%$ \\
\hline 18027 & IN & Daviess & rural & 673 & $28.6 \%$ & 429.49 & 1.567 & $83.1 \%$ \\
\hline 18033 & IN & DeKalb & rural & 640 & $28.6 \%$ & 362.82 & 1.763 & $43.2 \%$ \\
\hline 18029 & IN & Dearborn & urban & 1,082 & $28.5 \%$ & 305.03 & 3.546 & $65.7 \%$ \\
\hline 18031 & IN & Decatur & rural & 587 & $28.4 \%$ & 372.57 & 1.574 & $66.9 \%$ \\
\hline 18035 & IN & Delaware & urban & 2,690 & $28.5 \%$ & 392.12 & 6.861 & $73.6 \%$ \\
\hline 18037 & IN & Dubois & rural & 1,214 & $28.6 \%$ & 427.27 & 2.842 & $83.6 \%$ \\
\hline 18039 & IN & Elkhart & urban & 3,447 & $28.5 \%$ & 463.17 & 7.443 & $59.6 \%$ \\
\hline 18041 & IN & Fayette & rural & 644 & $28.5 \%$ & 215.01 & 2.995 & $76.6 \%$ \\
\hline 18043 & IN & Floyd & urban & 1,676 & $28.6 \%$ & 147.93 & 11.332 & $69.6 \%$ \\
\hline 18045 & IN & Fountain & rural & 558 & $28.5 \%$ & 395.66 & 1.411 & $76.6 \%$ \\
\hline 18047 & IN & Franklin & rural & 380 & $28.5 \%$ & 384.43 & 0.988 & $60.4 \%$ \\
\hline 18049 & IN & Fulton & rural & 364 & $28.3 \%$ & 368.39 & 0.988 & $50.5 \%$ \\
\hline 18051 & IN & Gibson & rural & 743 & $28.6 \%$ & 487.49 & 1.525 & $66.8 \%$ \\
\hline 18053 & IN & Grant & rural & 1,662 & $28.4 \%$ & 414.07 & 4.015 & $69.9 \%$ \\
\hline 18055 & IN & Greene & rural & 859 & $28.5 \%$ & 542.49 & 1.583 & $79.6 \%$ \\
\hline 18057 & IN & Hamilton & urban & 4,277 & $28.7 \%$ & 394.27 & 10.848 & $62.1 \%$ \\
\hline 18059 & IN & Hancock & urban & 1,425 & $28.7 \%$ & 306.02 & 4.656 & $60.1 \%$ \\
\hline 18061 & IN & Harrison & urban & 906 & $28.5 \%$ & 484.52 & 1.870 & $71.4 \%$ \\
\hline 18063 & IN & Hendricks & urban & 2,396 & $28.7 \%$ & 406.91 & 5.889 & $60.7 \%$ \\
\hline 18065 & IN & Henry & rural & 1,162 & $28.6 \%$ & 391.88 & 2.964 & $68.9 \%$ \\
\hline 18067 & IN & Howard & urban & 2,281 & $28.5 \%$ & 293.06 & 7.784 & $79.6 \%$ \\
\hline 18069 & IN & Huntington & rural & 661 & $28.4 \%$ & 382.65 & 1.727 & $44.4 \%$ \\
\hline 18071 & IN & Jackson & rural & 892 & $28.6 \%$ & 509.31 & 1.751 & $61.6 \%$ \\
\hline 18073 & IN & Jasper & urban & 872 & $28.7 \%$ & 559.62 & 1.559 & $79.7 \%$ \\
\hline 18075 & IN & Jay & rural & 474 & $28.6 \%$ & 383.91 & 1.234 & $63.8 \%$ \\
\hline
\end{tabular}




\begin{tabular}{|c|c|c|c|c|c|c|c|c|}
\hline 18081 & IN & Johnson & urban & 2,753 & $28.7 \%$ & 320.43 & 8.591 & $63.2 \%$ \\
\hline 18083 & IN & Knox & rural & 1,047 & $28.8 \%$ & 516.03 & 2.029 & $84.6 \%$ \\
\hline 18085 & IN & Kosciusko & rural & 1,182 & $28.4 \%$ & 531.38 & 2.224 & $48.0 \%$ \\
\hline 18087 & IN & LaGrange & rural & 541 & $28.5 \%$ & 379.62 & 1.426 & $53.4 \%$ \\
\hline 18091 & IN & LaPorte & urban & 2,789 & $28.6 \%$ & 598.30 & 4.662 & $79.7 \%$ \\
\hline 18089 & IN & Lake & urban & 9,669 & $28.4 \%$ & 498.96 & 19.378 & $74.5 \%$ \\
\hline 18093 & IN & Lawrence & rural & 1,176 & $28.6 \%$ & 449.17 & 2.618 & $74.8 \%$ \\
\hline 18095 & IN & Madison & urban & 3,040 & $28.5 \%$ & 451.92 & 6.726 & $67.0 \%$ \\
\hline 18097 & IN & Marion & urban & 12,752 & $28.4 \%$ & 396.30 & 32.177 & $58.0 \%$ \\
\hline 18099 & IN & Marshall & rural & 809 & $28.5 \%$ & 443.63 & 1.823 & $51.6 \%$ \\
\hline 18103 & IN & Miami & rural & 775 & $28.7 \%$ & 373.84 & 2.073 & $74.9 \%$ \\
\hline 18105 & IN & Monroe & urban & 2,429 & $28.6 \%$ & 394.51 & 6.157 & $69.8 \%$ \\
\hline 18107 & IN & Montgomery & rural & 861 & $28.8 \%$ & 504.61 & 1.706 & $62.9 \%$ \\
\hline 18109 & IN & Morgan & urban & 1,521 & $28.7 \%$ & 403.97 & 3.766 & $66.6 \%$ \\
\hline 18111 & IN & Newton & urban & 313 & $28.5 \%$ & 401.76 & 0.778 & $80.4 \%$ \\
\hline 18113 & IN & Noble & rural & 643 & $28.6 \%$ & 410.84 & 1.566 & $47.2 \%$ \\
\hline 18115 & IN & Ohio & urban & 141 & $28.6 \%$ & 86.14 & 1.634 & $71.7 \%$ \\
\hline 18117 & IN & Orange & rural & 503 & $28.7 \%$ & 398.39 & 1.264 & $72.9 \%$ \\
\hline 18119 & IN & Owen & urban & 460 & $28.6 \%$ & 385.29 & 1.193 & $68.5 \%$ \\
\hline 18125 & IN & Pike & rural & 313 & $28.3 \%$ & 334.24 & 0.937 & $72.7 \%$ \\
\hline 18127 & IN & Porter & urban & 3,540 & $28.7 \%$ & 418.15 & 8.465 & $74.0 \%$ \\
\hline 18129 & IN & Posey & urban & 537 & $28.6 \%$ & 409.57 & 1.311 & $69.0 \%$ \\
\hline 18131 & IN & Pulaski & rural & 355 & $28.3 \%$ & 433.65 & 0.818 & $77.5 \%$ \\
\hline 18133 & IN & Putnam & urban & 674 & $28.7 \%$ & 480.53 & 1.403 & $63.9 \%$ \\
\hline 18135 & IN & Randolph & rural & 730 & $28.7 \%$ & 452.38 & 1.613 & $76.8 \%$ \\
\hline 18137 & IN & Ripley & rural & 815 & $28.7 \%$ & 446.43 & 1.825 & $69.9 \%$ \\
\hline 18139 & IN & Rush & rural & 405 & $28.7 \%$ & 408.12 & 0.992 & $74.0 \%$ \\
\hline 18143 & IN & Scott & urban & 558 & $28.6 \%$ & 190.40 & 2.928 & $73.4 \%$ \\
\hline 18145 & IN & Shelby & urban & 838 & $28.6 \%$ & 411.15 & 2.038 & $66.3 \%$ \\
\hline 18147 & IN & Spencer & rural & 547 & $28.5 \%$ & 396.74 & 1.378 & $75.7 \%$ \\
\hline 18141 & IN & St. Joseph & urban & 4,973 & $28.4 \%$ & 457.85 & 10.863 & $57.5 \%$ \\
\hline 18149 & IN & Starke & rural & 553 & $28.6 \%$ & 309.13 & 1.788 & $77.5 \%$ \\
\hline 18151 & IN & Steuben & rural & 672 & $28.5 \%$ & 308.94 & 2.174 & $49.4 \%$ \\
\hline 18153 & IN & Sullivan & urban & 516 & $28.6 \%$ & 447.14 & 1.155 & $79.6 \%$ \\
\hline 18155 & IN & Switzerland & rural & 201 & $28.5 \%$ & 220.63 & 0.909 & $75.5 \%$ \\
\hline 18157 & IN & Tippecanoe & urban & 2,693 & $28.6 \%$ & 499.81 & 5.388 & $68.8 \%$ \\
\hline 18159 & IN & Tipton & rural & 402 & $28.8 \%$ & 260.54 & 1.541 & $76.1 \%$ \\
\hline 18167 & IN & Vigo & urban & 2,390 & $28.5 \%$ & 403.31 & 5.925 & $77.4 \%$ \\
\hline 18169 & IN & Wabash & rural & 663 & $28.4 \%$ & 412.43 & 1.608 & $50.5 \%$ \\
\hline 18171 & IN & Warren & rural & 171 & $29.0 \%$ & 364.68 & 0.468 & $79.8 \%$ \\
\hline 18173 & IN & Warrick & urban & 1,328 & $28.6 \%$ & 384.82 & 3.450 & $67.0 \%$ \\
\hline 18175 & IN & Washington & urban & 500 & $28.6 \%$ & 513.73 & 0.974 & $62.0 \%$ \\
\hline 18177 & IN & Wayne & rural & 1,910 & $28.7 \%$ & 401.74 & 4.754 & $79.9 \%$ \\
\hline 18179 & IN & Wells & urban & 443 & $28.4 \%$ & 368.09 & 1.202 & $49.4 \%$ \\
\hline 18181 & IN & White & rural & 765 & $28.7 \%$ & 505.12 & 1.514 & $76.2 \%$ \\
\hline 18183 & IN & Whitley & urban & 506 & $28.5 \%$ & 335.57 & 1.508 & $37.8 \%$ \\
\hline 20001 & KS & Allen & rural & 403 & $28.4 \%$ & 500.30 & 0.805 & $85.3 \%$ \\
\hline 20003 & KS & Anderson & rural & 275 & $28.6 \%$ & 579.65 & 0.474 & $88.6 \%$ \\
\hline 20005 & KS & Atchison & rural & 469 & $28.5 \%$ & 431.17 & 1.088 & $86.0 \%$ \\
\hline 20007 & KS & Barber & rural & 174 & $28.7 \%$ & $1,134.07$ & 0.153 & $88.6 \%$ \\
\hline 20009 & KS & Barton & rural & 817 & $28.4 \%$ & 895.40 & 0.912 & $90.7 \%$ \\
\hline 20011 & KS & Bourbon & rural & 411 & $28.4 \%$ & 635.47 & 0.647 & $82.3 \%$ \\
\hline 20013 & KS & Brown & rural & 317 & $28.7 \%$ & 570.87 & 0.556 & $91.0 \%$ \\
\hline 20015 & KS & Butler & urban & 1,424 & $28.4 \%$ & $1,429.86$ & 0.996 & $76.1 \%$ \\
\hline 20019 & KS & Chautauqua & rural & 132 & $28.4 \%$ & 638.88 & 0.207 & $92.3 \%$ \\
\hline 20021 & KS & Cherokee & rural & 549 & $28.6 \%$ & 587.57 & 0.935 & $82.4 \%$ \\
\hline 20023 & KS & Cheyenne & rural & 136 & $28.3 \%$ & $1,019.89$ & 0.134 & $92.0 \%$ \\
\hline 20027 & KS & Clay & rural & 302 & $28.6 \%$ & 645.30 & 0.468 & $87.7 \%$ \\
\hline 20029 & KS & Cloud & rural & 362 & $28.7 \%$ & 715.34 & 0.507 & $89.3 \%$ \\
\hline 20031 & KS & Coffey & rural & 290 & $28.6 \%$ & 626.95 & 0.462 & $88.0 \%$ \\
\hline 20035 & KS & Cowley & rural & 1,009 & $28.5 \%$ & $1,125.75$ & 0.897 & $88.7 \%$ \\
\hline 20037 & KS & Crawford & rural & 1,019 & $28.6 \%$ & 589.76 & 1.727 & $85.7 \%$ \\
\hline 20039 & KS & Decatur & rural & 134 & $28.6 \%$ & 893.52 & 0.150 & $88.2 \%$ \\
\hline 20041 & KS & Dickinson & rural & 633 & $28.5 \%$ & 847.07 & 0.747 & $90.2 \%$ \\
\hline 20043 & KS & Doniphan & urban & 244 & $28.5 \%$ & 393.41 & 0.620 & $89.6 \%$ \\
\hline 20045 & KS & Douglas & urban & 2,239 & $28.6 \%$ & 455.87 & 4.911 & $75.1 \%$ \\
\hline 20047 & KS & Edwards & rural & 109 & $28.8 \%$ & 621.89 & 0.176 & $89.6 \%$ \\
\hline 20049 & KS & Elk & rural & 124 & $28.2 \%$ & 644.26 & 0.192 & $91.8 \%$ \\
\hline 20051 & KS & Ellis & rural & 773 & $28.5 \%$ & 899.91 & 0.858 & $86.7 \%$ \\
\hline 20053 & KS & Ellsworth & rural & 203 & $28.6 \%$ & 715.85 & 0.283 & $91.7 \%$ \\
\hline 20055 & KS & Finney & rural & 624 & $28.3 \%$ & $1,301.97$ & 0.479 & $81.4 \%$ \\
\hline 20057 & KS & Ford & rural & 645 & $28.3 \%$ & $1,098.27$ & 0.587 & $85.7 \%$ \\
\hline 20059 & KS & Franklin & rural & 689 & $28.7 \%$ & 571.76 & 1.204 & $82.5 \%$ \\
\hline 20061 & KS & Geary & rural & 451 & $28.3 \%$ & 384.62 & 1.172 & $81.5 \%$ \\
\hline
\end{tabular}




\begin{tabular}{|c|c|c|c|c|c|c|c|c|}
\hline 20067 & KS & Grant & rural & 161 & $28.4 \%$ & 574.80 & 0.280 & $86.8 \%$ \\
\hline 20069 & KS & Gray & rural & 194 & $28.9 \%$ & 868.87 & 0.223 & $91.4 \%$ \\
\hline 20073 & KS & Greenwood & rural & 273 & $28.5 \%$ & $1,143.30$ & 0.239 & $91.0 \%$ \\
\hline 20077 & KS & Harper & rural & 209 & $28.6 \%$ & 801.27 & 0.261 & $93.6 \%$ \\
\hline 20079 & KS & Harvey & urban & 1,002 & $28.5 \%$ & 539.75 & 1.857 & $73.1 \%$ \\
\hline 20085 & KS & Jackson & urban & 386 & $28.8 \%$ & 656.22 & 0.589 & $84.8 \%$ \\
\hline 20087 & KS & Jefferson & urban & 536 & $28.6 \%$ & 532.57 & 1.006 & $80.2 \%$ \\
\hline 20089 & KS & Jewell & rural & 146 & $28.1 \%$ & 909.78 & 0.160 & $89.5 \%$ \\
\hline 20091 & KS & Johnson & urban & 10,033 & $28.6 \%$ & 473.37 & 21.195 & $57.9 \%$ \\
\hline 20095 & KS & Kingman & urban & 246 & $28.3 \%$ & 863.36 & 0.285 & $89.8 \%$ \\
\hline 20103 & KS & Leavenworth & urban & 1,491 & $28.6 \%$ & 462.83 & 3.222 & $76.1 \%$ \\
\hline 20105 & KS & Lincoln & rural & 118 & $29.2 \%$ & 719.40 & 0.164 & $89.8 \%$ \\
\hline 20107 & KS & Linn & urban & 262 & $28.5 \%$ & 594.06 & 0.442 & $67.1 \%$ \\
\hline 20111 & KS & Lyon & rural & 884 & $28.6 \%$ & 847.47 & 1.043 & $86.7 \%$ \\
\hline 20115 & KS & Marion & rural & 488 & $28.6 \%$ & 944.29 & 0.517 & $90.9 \%$ \\
\hline 20117 & KS & Marshall & rural & 403 & $28.6 \%$ & 900.18 & 0.448 & $89.9 \%$ \\
\hline 20113 & KS & McPherson & rural & 974 & $28.5 \%$ & 898.27 & 1.084 & $83.8 \%$ \\
\hline 20119 & KS & Meade & rural & 141 & $28.2 \%$ & 978.09 & 0.144 & $89.7 \%$ \\
\hline 20121 & KS & Miami & urban & 592 & $28.4 \%$ & 575.66 & 1.028 & $67.1 \%$ \\
\hline 20125 & KS & Montgomery & rural & 1,119 & $28.6 \%$ & 643.53 & 1.738 & $88.8 \%$ \\
\hline 20127 & KS & Morris & rural & 267 & $28.6 \%$ & 695.28 & 0.384 & $91.9 \%$ \\
\hline 20131 & KS & Nemaha & rural & 383 & $28.5 \%$ & 717.43 & 0.534 & $92.6 \%$ \\
\hline 20133 & KS & Neosho & rural & 510 & $28.6 \%$ & 571.47 & 0.893 & $89.1 \%$ \\
\hline 20135 & KS & Ness & rural & 125 & $28.6 \%$ & $1,074.75$ & 0.116 & $89.0 \%$ \\
\hline 20137 & KS & Norton & rural & 194 & $28.9 \%$ & 878.13 & 0.221 & $89.0 \%$ \\
\hline 20139 & KS & Osage & urban & 509 & $28.6 \%$ & 705.52 & 0.722 & $88.6 \%$ \\
\hline 20141 & KS & Osborne & rural & 165 & $28.7 \%$ & 892.50 & 0.185 & $90.7 \%$ \\
\hline 20143 & KS & Ottawa & rural & 205 & $28.8 \%$ & 720.73 & 0.285 & $92.0 \%$ \\
\hline 20145 & KS & Pawnee & rural & 118 & $27.9 \%$ & 754.26 & 0.156 & $93.6 \%$ \\
\hline 20147 & KS & Phillips & rural & 228 & $28.8 \%$ & 885.88 & 0.257 & $91.8 \%$ \\
\hline 20149 & KS & Pottawatomie & urban & 475 & $28.5 \%$ & 841.02 & 0.565 & $83.0 \%$ \\
\hline 20151 & KS & Pratt & rural & 307 & $28.5 \%$ & 735.04 & 0.418 & $93.4 \%$ \\
\hline 20153 & KS & Rawlins & rural & 120 & $28.3 \%$ & $1,069.42$ & 0.112 & $92.6 \%$ \\
\hline 20155 & KS & Reno & rural & 1,968 & $28.6 \%$ & $1,255.35$ & 1.568 & $85.7 \%$ \\
\hline 20157 & KS & Republic & rural & 249 & $28.6 \%$ & 717.37 & 0.347 & $93.2 \%$ \\
\hline 20159 & KS & Rice & rural & 334 & $28.7 \%$ & 726.24 & 0.459 & $90.2 \%$ \\
\hline 20161 & KS & Riley & urban & 1,186 & $28.7 \%$ & 609.77 & 1.945 & $80.9 \%$ \\
\hline 20163 & KS & Rooks & rural & 201 & $28.6 \%$ & 890.53 & 0.226 & $92.1 \%$ \\
\hline 20171 & KS & Scott & rural & 106 & $28.7 \%$ & 717.54 & 0.148 & $87.9 \%$ \\
\hline 20173 & KS & Sedgwick & urban & 9,679 & $28.5 \%$ & 997.51 & 9.703 & $70.0 \%$ \\
\hline 20175 & KS & Seward & rural & 320 & $28.4 \%$ & 639.50 & 0.500 & $83.9 \%$ \\
\hline 20177 & KS & Shawnee & urban & 4,936 & $28.6 \%$ & 544.02 & 9.073 & $82.1 \%$ \\
\hline 20181 & KS & Sherman & rural & 195 & $28.6 \%$ & $1,056.07$ & 0.185 & $91.7 \%$ \\
\hline 20183 & $\mathrm{KS}$ & Smith & rural & 198 & $28.3 \%$ & 895.46 & 0.221 & $91.1 \%$ \\
\hline 20185 & KS & Stafford & rural & 238 & $28.9 \%$ & 792.05 & 0.300 & $89.7 \%$ \\
\hline 20189 & KS & Stevens & rural & 120 & $28.7 \%$ & 727.29 & 0.165 & $85.6 \%$ \\
\hline 20191 & KS & Sumner & urban & 723 & $28.6 \%$ & $1,181.94$ & 0.612 & $79.7 \%$ \\
\hline 20193 & KS & Thomas & rural & 219 & $28.9 \%$ & $1,074.69$ & 0.204 & $90.1 \%$ \\
\hline 20195 & KS & Trego & rural & 106 & $28.5 \%$ & 889.48 & 0.119 & $92.8 \%$ \\
\hline 20197 & KS & Wabaunsee & urban & 226 & $28.5 \%$ & 794.30 & 0.285 & $88.3 \%$ \\
\hline 20201 & KS & Washington & rural & 230 & $28.1 \%$ & 894.76 & 0.257 & $89.4 \%$ \\
\hline 20205 & KS & Wilson & rural & 287 & $28.6 \%$ & 570.42 & 0.504 & $92.0 \%$ \\
\hline 20207 & KS & Woodson & rural & 123 & $28.8 \%$ & 497.82 & 0.247 & $88.4 \%$ \\
\hline 20209 & KS & Wyandotte & urban & 1,926 & $28.2 \%$ & 151.60 & 12.706 & $52.3 \%$ \\
\hline 21001 & $\mathrm{KY}$ & Adair & rural & 390 & $28.3 \%$ & 405.28 & 0.963 & $69.9 \%$ \\
\hline 21003 & $\mathrm{KY}$ & Allen & urban & 411 & $28.7 \%$ & 344.34 & 1.194 & $69.9 \%$ \\
\hline 21005 & $\mathrm{KY}$ & Anderson & rural & 320 & $28.2 \%$ & 201.83 & 1.584 & $49.7 \%$ \\
\hline 21007 & $\mathrm{KY}$ & Ballard & rural & 269 & $28.6 \%$ & 246.66 & 1.091 & $72.9 \%$ \\
\hline 21009 & $\mathrm{KY}$ & Barren & rural & 899 & $28.5 \%$ & 487.54 & 1.844 & $67.7 \%$ \\
\hline 21011 & $\mathrm{KY}$ & Bath & rural & 206 & $28.1 \%$ & 278.79 & 0.740 & $56.9 \%$ \\
\hline 21013 & KY & Bell & rural & 535 & $28.3 \%$ & 359.00 & 1.491 & $69.2 \%$ \\
\hline 21015 & $\mathrm{KY}$ & Boone & urban & 1,684 & $28.6 \%$ & 246.36 & 6.835 & $48.3 \%$ \\
\hline 21017 & $\mathrm{KY}$ & Bourbon & urban & 357 & $28.2 \%$ & 289.72 & 1.231 & $54.8 \%$ \\
\hline 21019 & KY & Boyd & urban & 974 & $28.5 \%$ & 159.86 & 6.095 & $66.2 \%$ \\
\hline 21021 & $\mathrm{KY}$ & Boyle & rural & 684 & $28.6 \%$ & 180.17 & 3.799 & $62.8 \%$ \\
\hline 21023 & KY & Bracken & urban & 182 & $28.2 \%$ & 205.61 & 0.884 & $60.3 \%$ \\
\hline 21025 & $\mathrm{KY}$ & Breathitt & rural & 203 & $28.2 \%$ & 492.41 & 0.413 & $60.7 \%$ \\
\hline 21027 & KY & Breckinridge & rural & 465 & $28.6 \%$ & 567.17 & 0.820 & $74.8 \%$ \\
\hline 21029 & $\mathrm{KY}$ & Bullitt & urban & 1,032 & $28.6 \%$ & 297.02 & 3.475 & $58.3 \%$ \\
\hline 21031 & KY & Butler & urban & 182 & $28.1 \%$ & 426.09 & 0.428 & $58.7 \%$ \\
\hline 21033 & $\mathrm{KY}$ & Caldwell & rural & 321 & $28.4 \%$ & 344.79 & 0.931 & $69.3 \%$ \\
\hline 21035 & $\mathrm{KY}$ & Calloway & rural & 803 & $28.5 \%$ & 385.02 & 2.086 & $64.6 \%$ \\
\hline 21037 & KY & Campbell & urban & 1,314 & $28.5 \%$ & 151.31 & 8.682 & $48.0 \%$ \\
\hline 21039 & $\mathrm{KY}$ & Carlisle & rural & 132 & $27.9 \%$ & 189.43 & 0.694 & $70.7 \%$ \\
\hline 21041 & $\mathrm{KY}$ & Carroll & rural & 212 & $28.5 \%$ & 128.57 & 1.650 & $64.9 \%$ \\
\hline
\end{tabular}




\begin{tabular}{|c|c|c|c|c|c|c|c|c|}
\hline 21047 & $\mathrm{KY}$ & Christian & urban & 1,020 & $28.4 \%$ & 717.50 & 1.421 & $67.5 \%$ \\
\hline 21049 & $\mathrm{KY}$ & Clark & urban & 667 & $28.4 \%$ & 252.46 & 2.643 & $57.7 \%$ \\
\hline 21051 & $\mathrm{KY}$ & Clay & rural & 328 & $28.3 \%$ & 469.25 & 0.699 & $68.8 \%$ \\
\hline 21053 & KY & Clinton & rural & 258 & $28.6 \%$ & 197.25 & 1.309 & $71.7 \%$ \\
\hline 21055 & KY & Crittenden & rural & 226 & $29.1 \%$ & 359.95 & 0.628 & $74.9 \%$ \\
\hline 21057 & $\mathrm{KY}$ & Cumberland & rural & 181 & $28.5 \%$ & 305.18 & 0.595 & $68.0 \%$ \\
\hline 21059 & $\mathrm{KY}$ & Daviess & urban & 2,102 & $28.5 \%$ & 458.35 & 4.587 & $68.2 \%$ \\
\hline 21061 & $\mathrm{KY}$ & Edmonson & urban & 190 & $28.4 \%$ & 302.88 & 0.627 & $67.6 \%$ \\
\hline 21063 & $\mathrm{KY}$ & Elliott & rural & 246 & $28.5 \%$ & 234.32 & 1.048 & $60.7 \%$ \\
\hline 21065 & $\mathrm{KY}$ & Estill & rural & 299 & $28.7 \%$ & 253.08 & 1.182 & $65.3 \%$ \\
\hline 21069 & $\mathrm{KY}$ & Fleming & rural & 300 & $28.4 \%$ & 348.54 & 0.862 & $60.8 \%$ \\
\hline 21071 & $\mathrm{KY}$ & Floyd & rural & 630 & $28.7 \%$ & 393.34 & 1.601 & $61.6 \%$ \\
\hline 21073 & $\mathrm{KY}$ & Franklin & rural & 896 & $28.3 \%$ & 207.75 & 4.315 & $45.5 \%$ \\
\hline 21075 & $\mathrm{KY}$ & Fulton & rural & 208 & $28.3 \%$ & 205.50 & 1.011 & $77.4 \%$ \\
\hline 21077 & $\mathrm{KY}$ & Gallatin & urban & 107 & $28.1 \%$ & 101.23 & 1.055 & $55.8 \%$ \\
\hline 21079 & $\mathrm{KY}$ & Garrard & rural & 327 & $28.4 \%$ & 230.08 & 1.422 & $62.0 \%$ \\
\hline 21081 & $\mathrm{KY}$ & Grant & urban & 384 & $28.5 \%$ & 257.96 & 1.488 & $51.7 \%$ \\
\hline 21083 & $\mathrm{KY}$ & Graves & rural & 875 & $28.4 \%$ & 551.74 & 1.586 & $65.1 \%$ \\
\hline 21085 & $\mathrm{KY}$ & Grayson & rural & 660 & $28.7 \%$ & 496.70 & 1.329 & $72.9 \%$ \\
\hline 21091 & $\mathrm{KY}$ & Hancock & urban & 214 & $28.6 \%$ & 187.65 & 1.143 & $76.0 \%$ \\
\hline 21093 & $\mathrm{KY}$ & Hardin & urban & 2,026 & $28.5 \%$ & 623.28 & 3.251 & $74.5 \%$ \\
\hline 21095 & $\mathrm{KY}$ & Harlan & rural & 551 & $28.4 \%$ & 465.83 & 1.182 & $68.6 \%$ \\
\hline 21097 & $\mathrm{KY}$ & Harrison & rural & 380 & $28.4 \%$ & 306.36 & 1.240 & $63.2 \%$ \\
\hline 21099 & $\mathrm{KY}$ & Hart & rural & 362 & $28.5 \%$ & 412.09 & 0.878 & $71.8 \%$ \\
\hline 21101 & KY & Henderson & urban & 919 & $28.4 \%$ & 436.67 & 2.105 & $62.4 \%$ \\
\hline 21103 & $\mathrm{KY}$ & Henry & urban & 315 & $28.4 \%$ & 286.28 & 1.099 & $53.5 \%$ \\
\hline 21107 & $\mathrm{KY}$ & Hopkins & rural & 1,081 & $28.4 \%$ & 542.00 & 1.995 & $68.5 \%$ \\
\hline 21109 & $\mathrm{KY}$ & Jackson & rural & 243 & $28.4 \%$ & 345.20 & 0.703 & $67.8 \%$ \\
\hline 21111 & $\mathrm{KY}$ & Jefferson & urban & 13,846 & $28.5 \%$ & 380.42 & 36.396 & $58.7 \%$ \\
\hline 21113 & $\mathrm{KY}$ & Jessamine & urban & 747 & $28.3 \%$ & 172.12 & 4.342 & $56.0 \%$ \\
\hline 21115 & KY & Johnson & rural & 410 & $28.7 \%$ & 261.95 & 1.565 & $63.0 \%$ \\
\hline 21117 & KY & Kenton & urban & 2,272 & $28.5 \%$ & 160.25 & 14.176 & $51.8 \%$ \\
\hline 21119 & KY & Knott & rural & 207 & $28.3 \%$ & 351.52 & 0.590 & $62.1 \%$ \\
\hline 21121 & $\mathrm{KY}$ & Knox & rural & 425 & $28.6 \%$ & 386.30 & 1.099 & $68.9 \%$ \\
\hline 21123 & $\mathrm{KY}$ & Larue & urban & 325 & $28.6 \%$ & 261.52 & 1.244 & $70.0 \%$ \\
\hline 21125 & $\mathrm{KY}$ & Laurel & rural & 929 & $28.5 \%$ & 433.95 & 2.141 & $65.5 \%$ \\
\hline 21127 & KY & Lawrence & rural & 316 & $28.4 \%$ & 415.60 & 0.761 & $72.5 \%$ \\
\hline 21135 & $\mathrm{KY}$ & Lewis & rural & 261 & $28.4 \%$ & 482.84 & 0.540 & $66.3 \%$ \\
\hline 21137 & $\mathrm{KY}$ & Lincoln & rural & 555 & $28.5 \%$ & 334.09 & 1.663 & $62.7 \%$ \\
\hline 21139 & $\mathrm{KY}$ & Livingston & rural & 287 & $28.8 \%$ & 313.13 & 0.916 & $73.6 \%$ \\
\hline 21141 & $\mathrm{KY}$ & Logan & rural & 650 & $28.7 \%$ & 552.13 & 1.178 & $71.3 \%$ \\
\hline 21143 & $\mathrm{KY}$ & Lyon & rural & 229 & $28.9 \%$ & 213.84 & 1.070 & $63.8 \%$ \\
\hline 21151 & $\mathrm{KY}$ & Madison & rural & 1,232 & $28.4 \%$ & 437.29 & 2.817 & $54.6 \%$ \\
\hline 21153 & $\mathrm{KY}$ & Magoffin & rural & 204 & $28.6 \%$ & 308.44 & 0.661 & $65.8 \%$ \\
\hline 21155 & $\mathrm{KY}$ & Marion & rural & 414 & $28.3 \%$ & 343.01 & 1.208 & $69.4 \%$ \\
\hline 21157 & $\mathrm{KY}$ & Marshall & rural & 922 & $28.8 \%$ & 301.25 & 3.061 & $69.7 \%$ \\
\hline 21159 & $\mathrm{KY}$ & Martin & rural & 173 & $28.8 \%$ & 229.60 & 0.756 & $65.4 \%$ \\
\hline 21161 & $\mathrm{KY}$ & Mason & rural & 362 & $28.2 \%$ & 240.13 & 1.507 & $62.2 \%$ \\
\hline 21145 & $\mathrm{KY}$ & McCracken & rural & 1,683 & $28.5 \%$ & 248.74 & 6.767 & $70.3 \%$ \\
\hline 21147 & $\mathrm{KY}$ & McCreary & rural & 273 & $28.5 \%$ & 426.80 & 0.640 & $66.0 \%$ \\
\hline 21149 & $\mathrm{KY}$ & McLean & urban & 227 & $28.2 \%$ & 252.47 & 0.899 & $65.2 \%$ \\
\hline 21163 & $\mathrm{KY}$ & Meade & urban & 402 & $28.5 \%$ & 305.42 & 1.315 & $70.2 \%$ \\
\hline 21165 & $\mathrm{KY}$ & Menifee & rural & 144 & $28.3 \%$ & 203.58 & 0.707 & $54.9 \%$ \\
\hline 21167 & $\mathrm{KY}$ & Mercer & rural & 481 & $28.5 \%$ & 248.80 & 1.935 & $60.0 \%$ \\
\hline 21169 & $\mathrm{KY}$ & Metcalfe & rural & 212 & $28.3 \%$ & 289.65 & 0.733 & $56.9 \%$ \\
\hline 21171 & $\mathrm{KY}$ & Monroe & rural & 307 & $28.6 \%$ & 329.37 & 0.931 & $73.1 \%$ \\
\hline 21173 & KY & Montgomery & rural & 460 & $28.3 \%$ & 197.37 & 2.331 & $57.2 \%$ \\
\hline 21175 & $\mathrm{KY}$ & Morgan & rural & 208 & $28.6 \%$ & 381.13 & 0.545 & $57.0 \%$ \\
\hline 21177 & $\mathrm{KY}$ & Muhlenberg & rural & 715 & $28.8 \%$ & 467.08 & 1.531 & $73.1 \%$ \\
\hline 21179 & $\mathrm{KY}$ & Nelson & rural & 970 & $28.6 \%$ & 417.51 & 2.323 & $68.7 \%$ \\
\hline 21181 & $\mathrm{KY}$ & Nicholas & rural & 154 & $28.5 \%$ & 195.17 & 0.789 & $65.7 \%$ \\
\hline 21183 & KY & Ohio & rural & 412 & $28.5 \%$ & 587.27 & 0.701 & $64.2 \%$ \\
\hline 21185 & KY & Oldham & urban & 789 & $28.6 \%$ & 187.22 & 4.215 & $54.8 \%$ \\
\hline 21187 & $\mathrm{KY}$ & Owen & rural & 156 & $28.2 \%$ & 351.10 & 0.444 & $59.4 \%$ \\
\hline 21189 & $\mathrm{KY}$ & Owsley & rural & 102 & $28.0 \%$ & 197.41 & 0.515 & $67.6 \%$ \\
\hline 21191 & $\mathrm{KY}$ & Pendleton & urban & 222 & $28.3 \%$ & 277.16 & 0.802 & $53.9 \%$ \\
\hline 21193 & KY & Perry & rural & 569 & $28.8 \%$ & 339.67 & 1.674 & $67.6 \%$ \\
\hline 21195 & $\mathrm{KY}$ & Pike & rural & 1,068 & $28.4 \%$ & 786.83 & 1.357 & $65.9 \%$ \\
\hline 21197 & $\mathrm{KY}$ & Powell & rural & 200 & $28.7 \%$ & 178.98 & 1.120 & $57.0 \%$ \\
\hline 21199 & KY & Pulaski & rural & 1,502 & $28.7 \%$ & 658.41 & 2.282 & $61.7 \%$ \\
\hline 21203 & $\mathrm{KY}$ & Rockcastle & rural & 323 & $28.8 \%$ & 316.54 & 1.021 & $67.7 \%$ \\
\hline 21205 & KY & Rowan & rural & 307 & $28.5 \%$ & 279.80 & 1.095 & $48.0 \%$ \\
\hline 21207 & KY & Russell & rural & 433 & $28.4 \%$ & 253.66 & 1.706 & $68.5 \%$ \\
\hline 21209 & KY & Scott & urban & 636 & $28.5 \%$ & 281.77 & 2.256 & $51.8 \%$ \\
\hline
\end{tabular}




\begin{tabular}{|c|c|c|c|c|c|c|c|c|}
\hline 21215 & $\mathrm{KY}$ & Spencer & urban & 266 & $28.3 \%$ & 186.68 & 1.424 & $59.2 \%$ \\
\hline 21217 & $\mathrm{KY}$ & Taylor & rural & 669 & $28.6 \%$ & 266.33 & 2.511 & $69.0 \%$ \\
\hline 21219 & $\mathrm{KY}$ & Todd & rural & 229 & $28.6 \%$ & 374.50 & 0.612 & $72.1 \%$ \\
\hline 21221 & $\mathrm{KY}$ & Trigg & urban & 371 & $28.5 \%$ & 441.43 & 0.840 & $63.8 \%$ \\
\hline 21223 & $\mathrm{KY}$ & Trimble & urban & 171 & $28.6 \%$ & 151.65 & 1.126 & $65.6 \%$ \\
\hline 21225 & $\mathrm{KY}$ & Union & rural & 326 & $28.2 \%$ & 342.85 & 0.951 & $70.0 \%$ \\
\hline 21227 & $\mathrm{KY}$ & Warren & urban & 2,088 & $28.3 \%$ & 541.60 & 3.855 & $64.8 \%$ \\
\hline 21229 & $\mathrm{KY}$ & Washington & rural & 255 & $28.4 \%$ & 297.27 & 0.856 & $68.4 \%$ \\
\hline 21231 & $\mathrm{KY}$ & Wayne & rural & 413 & $28.7 \%$ & 458.17 & 0.902 & $63.6 \%$ \\
\hline 21233 & $\mathrm{KY}$ & Webster & rural & 296 & $28.2 \%$ & 331.94 & 0.892 & $63.8 \%$ \\
\hline 21237 & $\mathrm{KY}$ & Wolfe & rural & 144 & $28.5 \%$ & 222.17 & 0.650 & $60.1 \%$ \\
\hline 21239 & $\mathrm{KY}$ & Woodford & urban & 466 & $28.5 \%$ & 188.78 & 2.467 & $50.6 \%$ \\
\hline 22001 & LA & Acadia & urban & 1,157 & $28.4 \%$ & 655.12 & 1.766 & $82.2 \%$ \\
\hline 22003 & LA & Allen & rural & 435 & $28.4 \%$ & 761.85 & 0.571 & $78.2 \%$ \\
\hline 22005 & LA & Ascension & urban & 830 & $28.4 \%$ & 289.98 & 2.863 & $31.7 \%$ \\
\hline 22007 & LA & Assumption & rural & 343 & $28.2 \%$ & 338.66 & 1.013 & $62.7 \%$ \\
\hline 22009 & LA & Avoyelles & rural & 908 & $28.3 \%$ & 832.43 & 1.091 & $77.9 \%$ \\
\hline 22011 & LA & Beauregard & rural & 826 & $28.8 \%$ & $1,157.34$ & 0.714 & $80.0 \%$ \\
\hline 22013 & LA & Bienville & rural & 346 & $28.1 \%$ & 811.27 & 0.426 & $72.5 \%$ \\
\hline 22019 & LA & Calcasieu & urban & 4,125 & $28.4 \%$ & $1,063.66$ & 3.878 & $75.5 \%$ \\
\hline 22021 & LA & Caldwell & rural & 231 & $28.3 \%$ & 529.42 & 0.437 & $70.7 \%$ \\
\hline 22025 & LA & Catahoula & rural & 245 & $28.5 \%$ & 708.03 & 0.346 & $76.8 \%$ \\
\hline 22027 & LA & Claiborne & rural & 351 & $28.1 \%$ & 754.88 & 0.465 & $76.8 \%$ \\
\hline 22029 & LA & Concordia & rural & 417 & $28.3 \%$ & 696.92 & 0.599 & $74.8 \%$ \\
\hline 22031 & LA & De Soto & urban & 620 & $28.2 \%$ & 875.58 & 0.708 & $75.9 \%$ \\
\hline 22033 & LA & East Baton Rouge & urban & 5,245 & $28.4 \%$ & 455.37 & 11.518 & $43.8 \%$ \\
\hline 22035 & LA & East Carroll & rural & 127 & $28.2 \%$ & 420.7 & 0.303 & $77.8 \%$ \\
\hline 22037 & LA & East Feliciana & urban & 309 & $28.2 \%$ & 453.41 & 0.681 & $48.5 \%$ \\
\hline 22039 & LA & Evangeline & rural & 682 & $28.4 \%$ & 662.38 & 1.029 & $83.4 \%$ \\
\hline 22041 & LA & Franklin & rural & 461 & $28.2 \%$ & 624.59 & 0.738 & $75.0 \%$ \\
\hline 22043 & LA & Grant & urban & 417 & $29.0 \%$ & 643.03 & 0.649 & $73.3 \%$ \\
\hline 22045 & LA & Iberia & urban & 1,460 & $28.4 \%$ & 574.11 & 2.544 & $81.0 \%$ \\
\hline 22047 & LA & Iberville & urban & 350 & $28.2 \%$ & 618.63 & 0.566 & $37.1 \%$ \\
\hline 22049 & LA & Jackson & rural & 389 & $28.5 \%$ & 569.18 & 0.683 & $70.8 \%$ \\
\hline 22051 & LA & Jefferson & urban & 4,219 & $28.5 \%$ & 295.63 & 14.271 & $27.9 \%$ \\
\hline 22053 & LA & Jefferson Davis & rural & 665 & $28.5 \%$ & 651.33 & 1.021 & $84.6 \%$ \\
\hline 22059 & LA & LaSalle & rural & 326 & $28.6 \%$ & 624.68 & 0.522 & $77.6 \%$ \\
\hline 22063 & LA & Livingston & urban & 1,196 & $28.5 \%$ & 648.17 & 1.845 & $35.8 \%$ \\
\hline 22065 & LA & Madison & rural & 209 & $27.8 \%$ & 624.44 & 0.335 & $79.1 \%$ \\
\hline 22067 & LA & Morehouse & rural & 546 & $28.4 \%$ & 794.93 & 0.687 & $65.5 \%$ \\
\hline 22069 & LA & Natchitoches & rural & 785 & $28.3 \%$ & $1,252.25$ & 0.627 & $74.9 \%$ \\
\hline 22071 & LA & Orleans & urban & 3,300 & $28.3 \%$ & 169.42 & 19.478 & $36.2 \%$ \\
\hline 22073 & LA & Ouachita & urban & 2,783 & $28.5 \%$ & 610.41 & 4.559 & $67.2 \%$ \\
\hline 22075 & LA & Plaquemines & urban & 171 & $27.9 \%$ & 779.91 & 0.219 & $29.5 \%$ \\
\hline 22077 & LA & Pointe Coupee & urban & 324 & $28.2 \%$ & 557.35 & 0.582 & $44.4 \%$ \\
\hline 22079 & LA & Rapides & urban & 2,822 & $28.5 \%$ & $1,317.96$ & 2.141 & $74.5 \%$ \\
\hline 22081 & LA & Red River & rural & 178 & $28.9 \%$ & 389.09 & 0.456 & $70.6 \%$ \\
\hline 22083 & LA & Richland & rural & 436 & $28.6 \%$ & 559.04 & 0.781 & $70.5 \%$ \\
\hline 22085 & LA & Sabine & rural & 624 & $28.6 \%$ & 866.66 & 0.720 & $78.6 \%$ \\
\hline 22087 & LA & St. Bernard & urban & 307 & $28.5 \%$ & 377.52 & 0.812 & $37.0 \%$ \\
\hline 22089 & LA & St. Charles & urban & 393 & $28.3 \%$ & 279.08 & 1.409 & $29.4 \%$ \\
\hline 22093 & LA & St. James & urban & 276 & $28.5 \%$ & 241.54 & 1.141 & $43.7 \%$ \\
\hline 22095 & LA & St. John the Baptist & urban & 382 & $28.4 \%$ & 213.07 & 1.791 & $34.1 \%$ \\
\hline 22097 & LA & St. Landry & rural & 1,971 & $28.3 \%$ & 923.88 & 2.133 & $80.4 \%$ \\
\hline 22099 & LA & St. Martin & urban & 922 & $28.4 \%$ & 737.65 & 1.250 & $78.2 \%$ \\
\hline 22101 & LA & St. Mary & rural & 1,012 & $28.3 \%$ & 555.38 & 1.823 & $72.9 \%$ \\
\hline 22103 & LA & St. Tammany & urban & 3,158 & $28.5 \%$ & 845.55 & 3.735 & $37.1 \%$ \\
\hline 22105 & LA & Tangipahoa & urban & 1,724 & $28.3 \%$ & 791.27 & 2.179 & $52.2 \%$ \\
\hline 22107 & LA & Tensas & rural & 129 & $28.2 \%$ & 602.78 & 0.214 & $75.9 \%$ \\
\hline 22109 & LA & Terrebonne & urban & 2,107 & $28.5 \%$ & $1,231.81$ & 1.711 & $73.2 \%$ \\
\hline 22111 & LA & Union & urban & 549 & $28.4 \%$ & 876.99 & 0.626 & $71.0 \%$ \\
\hline 22113 & LA & Vermilion & urban & 1,336 & $28.5 \%$ & $1,173.20$ & 1.139 & $85.7 \%$ \\
\hline 22115 & LA & Vernon & rural & 737 & $28.6 \%$ & $1,327.91$ & 0.555 & $82.7 \%$ \\
\hline 22117 & LA & Washington & rural & 784 & $28.3 \%$ & 669.52 & 1.171 & $60.5 \%$ \\
\hline 22119 & LA & Webster & urban & 1,046 & $28.3 \%$ & 593.03 & 1.764 & $71.9 \%$ \\
\hline 22121 & LA & West Baton Rouge & urban & 226 & $28.2 \%$ & 192.39 & 1.175 & $35.2 \%$ \\
\hline 22123 & LA & West Carroll & rural & 285 & $28.9 \%$ & 359.65 & 0.793 & $81.0 \%$ \\
\hline 22125 & LA & West Feliciana & urban & 173 & $28.2 \%$ & 403.21 & 0.428 & $53.8 \%$ \\
\hline 22127 & LA & Winn & rural & 303 & $28.5 \%$ & 950.09 & 0.319 & $76.8 \%$ \\
\hline 25001 & $\mathrm{MA}$ & Barnstable & urban & 11,052 & $28.7 \%$ & 393.72 & 28.070 & $78.4 \%$ \\
\hline 25003 & MA & Berkshire & urban & 4,609 & $28.6 \%$ & 926.82 & 4.973 & $84.8 \%$ \\
\hline 25005 & MA & Bristol & urban & 11,768 & $28.7 \%$ & 553.10 & 21.276 & $68.7 \%$ \\
\hline 25007 & MA & Dukes & rural & 763 & $28.6 \%$ & 103.25 & 7.391 & $88.7 \%$ \\
\hline 25009 & MA & Essex & urban & 16,966 & $28.6 \%$ & 492.56 & 34.445 & $66.8 \%$ \\
\hline
\end{tabular}




\begin{tabular}{|c|c|c|c|c|c|c|c|c|}
\hline 25015 & $\mathrm{MA}$ & Hampshire & urban & 3,607 & $28.6 \%$ & 527.26 & 6.841 & $66.3 \%$ \\
\hline 25017 & $\mathrm{MA}$ & Middlesex & urban & 28,457 & $28.6 \%$ & 817.82 & 34.796 & $58.5 \%$ \\
\hline 25019 & $\mathrm{MA}$ & Nantucket & rural & 322 & $28.6 \%$ & 44.97 & 7.165 & $88.5 \%$ \\
\hline 25021 & MA & Norfolk & urban & 14,396 & $28.6 \%$ & 396.11 & 36.343 & $64.0 \%$ \\
\hline 25023 & MA & Plymouth & urban & 12,434 & $28.7 \%$ & 659.08 & 18.866 & $70.4 \%$ \\
\hline 25025 & $\mathrm{MA}$ & Suffolk & urban & 9,519 & $28.3 \%$ & 58.15 & 163.704 & $63.0 \%$ \\
\hline 25027 & $\mathrm{MA}$ & Worcester & urban & 12,280 & $28.6 \%$ & $1,510.77$ & 8.128 & $47.7 \%$ \\
\hline 24001 & $\mathrm{MD}$ & Allegany & urban & 2,271 & $28.6 \%$ & 424.16 & 5.355 & $88.2 \%$ \\
\hline 24003 & $\mathrm{MD}$ & Anne Arundel & urban & 11,727 & $28.6 \%$ & 414.9 & 28.266 & $78.0 \%$ \\
\hline 24005 & $\mathrm{MD}$ & Baltimore & urban & 17,432 & $28.4 \%$ & 598.30 & 29.135 & $76.5 \%$ \\
\hline 24009 & $M D$ & Calvert & urban & 1,791 & $28.4 \%$ & 213.15 & 8.405 & $80.5 \%$ \\
\hline 24011 & $\mathrm{MD}$ & Caroline & rural & 809 & $28.5 \%$ & 319.42 & 2.531 & $87.0 \%$ \\
\hline 24013 & $\mathrm{MD}$ & Carroll & urban & 4,491 & $28.7 \%$ & 447.59 & 10.033 & $80.2 \%$ \\
\hline 24015 & $\mathrm{MD}$ & Cecil & urban & 2,199 & $28.6 \%$ & 346.27 & 6.350 & $84.3 \%$ \\
\hline 24017 & $\mathrm{MD}$ & Charles & urban & 2,063 & $28.5 \%$ & 457.75 & 4.508 & $75.2 \%$ \\
\hline 24019 & $\mathrm{MD}$ & Dorchester & rural & 963 & $28.5 \%$ & 540.77 & 1.780 & $87.3 \%$ \\
\hline 24021 & $\mathrm{MD}$ & Frederick & urban & 4,434 & $28.7 \%$ & 660.22 & 6.715 & $76.7 \%$ \\
\hline 24023 & $\mathrm{MD}$ & Garrett & rural & 855 & $28.7 \%$ & 647.10 & 1.321 & $86.2 \%$ \\
\hline 24025 & $\mathrm{MD}$ & Harford & urban & 5,669 & $28.7 \%$ & 437.09 & 12.970 & $80.2 \%$ \\
\hline 24031 & $\mathrm{MD}$ & Montgomery & urban & 18,453 & $28.6 \%$ & 491.25 & 37.562 & $66.5 \%$ \\
\hline 24033 & $\mathrm{MD}$ & Prince George's & urban & 10,991 & $28.3 \%$ & 482.69 & 22.770 & $60.2 \%$ \\
\hline 24035 & $\mathrm{MD}$ & Queen Anne's & urban & 1,258 & $28.8 \%$ & 371.91 & 3.381 & $85.4 \%$ \\
\hline 24039 & $\mathrm{MD}$ & Somerset & urban & 596 & $28.4 \%$ & 319.72 & 1.864 & $87.4 \%$ \\
\hline 24037 & $\mathrm{MD}$ & St. Mary's & urban & 1,821 & $28.6 \%$ & 357.18 & 5.098 & $83.2 \%$ \\
\hline 24041 & $\mathrm{MD}$ & Talbot & rural & 1,710 & $28.7 \%$ & 268.54 & 6.367 & $88.1 \%$ \\
\hline 24043 & $\mathrm{MD}$ & Washington & urban & 3,240 & $28.6 \%$ & 457.78 & 7.079 & $77.8 \%$ \\
\hline 24045 & $\mathrm{MD}$ & Wicomico & urban & 2,337 & $28.4 \%$ & 374.44 & 6.241 & $86.5 \%$ \\
\hline 24047 & $\mathrm{MD}$ & Worcester & urban & 2,199 & $28.7 \%$ & 468.28 & 4.695 & $89.6 \%$ \\
\hline 23001 & $\mathrm{ME}$ & Androscoggin & urban & 2,201 & $28.6 \%$ & 467.93 & 4.704 & $57.0 \%$ \\
\hline 23003 & $\mathrm{ME}$ & Aroostook & rural & 2,216 & $28.5 \%$ & $6,671.33$ & 0.332 & $75.6 \%$ \\
\hline 23005 & $\mathrm{ME}$ & Cumberland & urban & 6,290 & $28.6 \%$ & 835.24 & 7.531 & $57.4 \%$ \\
\hline 23007 & $\mathrm{ME}$ & Franklin & rural & 740 & $28.7 \%$ & $1,696.61$ & 0.436 & $60.6 \%$ \\
\hline 23009 & $\mathrm{ME}$ & Hancock & rural & 1,843 & $28.8 \%$ & $1,586.89$ & 1.161 & $70.7 \%$ \\
\hline 23011 & $\mathrm{ME}$ & Kennebec & rural & 2,655 & $28.6 \%$ & 867.52 & 3.060 & $55.2 \%$ \\
\hline 23013 & $\mathrm{ME}$ & Knox & rural & 1,373 & $28.5 \%$ & 365.13 & 3.760 & $67.2 \%$ \\
\hline 23015 & $\mathrm{ME}$ & Lincoln & rural & 1,229 & $28.7 \%$ & 455.82 & 2.697 & $62.4 \%$ \\
\hline 23017 & $\mathrm{ME}$ & Oxford & rural & 1,489 & $28.6 \%$ & $2,076.84$ & 0.717 & $66.4 \%$ \\
\hline 23025 & $\mathrm{ME}$ & Somerset & rural & 1,374 & $28.4 \%$ & $3,924.40$ & 0.350 & $70.3 \%$ \\
\hline 23027 & $\mathrm{ME}$ & Waldo & rural & 1,087 & $28.5 \%$ & 729.92 & 1.489 & $63.8 \%$ \\
\hline 23029 & $\mathrm{ME}$ & Washington & rural & 1,149 & $28.6 \%$ & $2,562.66$ & 0.448 & $77.7 \%$ \\
\hline 23031 & $\mathrm{ME}$ & York & urban & 5,238 & $28.7 \%$ & 990.71 & 5.287 & $65.3 \%$ \\
\hline 26001 & $\mathrm{MI}$ & Alcona & rural & 442 & $28.7 \%$ & 674.59 & 0.655 & $66.5 \%$ \\
\hline 26003 & $\mathrm{MI}$ & Alger & rural & 314 & $28.3 \%$ & 915.07 & 0.344 & $69.3 \%$ \\
\hline 26005 & $\mathrm{MI}$ & Allegan & rural & 1,280 & $28.4 \%$ & 825.23 & 1.551 & $39.0 \%$ \\
\hline 26007 & $\mathrm{MI}$ & Alpena & rural & 1,000 & $28.6 \%$ & 571.86 & 1.748 & $72.0 \%$ \\
\hline 26009 & $\mathrm{MI}$ & Antrim & rural & 648 & $28.7 \%$ & 475.70 & 1.363 & $58.7 \%$ \\
\hline 26011 & $\mathrm{MI}$ & Arenac & rural & 466 & $28.3 \%$ & 363.19 & 1.283 & $62.5 \%$ \\
\hline 26013 & $\mathrm{MI}$ & Baraga & rural & 231 & $28.7 \%$ & 898.26 & 0.257 & $67.8 \%$ \\
\hline 26015 & $\mathrm{MI}$ & Barry & urban & 864 & $28.6 \%$ & 553.09 & 1.562 & $47.3 \%$ \\
\hline 26017 & $\mathrm{MI}$ & Bay & urban & 2,233 & $28.5 \%$ & 442.30 & 5.048 & $59.1 \%$ \\
\hline 26019 & $\mathrm{MI}$ & Benzie & rural & 475 & $28.6 \%$ & 319.70 & 1.487 & $55.9 \%$ \\
\hline 26021 & $\mathrm{MI}$ & Berrien & urban & 3,965 & $28.5 \%$ & 567.75 & 6.984 & $63.4 \%$ \\
\hline 26023 & $\mathrm{MI}$ & Branch & rural & 985 & $28.7 \%$ & 506.37 & 1.946 & $69.0 \%$ \\
\hline 26025 & $\mathrm{MI}$ & Calhoun & urban & 2,985 & $28.5 \%$ & 706.23 & 4.227 & $68.2 \%$ \\
\hline 26027 & $\mathrm{MI}$ & Cass & urban & 812 & $28.4 \%$ & 490.06 & 1.657 & $61.6 \%$ \\
\hline 26029 & $\mathrm{MI}$ & Charlevoix & rural & 760 & $28.5 \%$ & 416.34 & 1.825 & $60.1 \%$ \\
\hline 26031 & $\mathrm{MI}$ & Cheboygan & rural & 746 & $28.7 \%$ & 715.26 & 1.043 & $65.1 \%$ \\
\hline 26033 & $\mathrm{MI}$ & Chippewa & rural & 764 & $28.6 \%$ & $1,558.42$ & 0.490 & $63.6 \%$ \\
\hline 26035 & $\mathrm{MI}$ & Clare & rural & 812 & $28.6 \%$ & 564.32 & 1.439 & $64.6 \%$ \\
\hline 26037 & $\mathrm{MI}$ & Clinton & urban & 1,101 & $28.5 \%$ & 566.41 & 1.943 & $56.7 \%$ \\
\hline 26039 & $\mathrm{MI}$ & Crawford & rural & 317 & $28.7 \%$ & 556.28 & 0.570 & $64.3 \%$ \\
\hline 26041 & $\mathrm{MI}$ & Delta & rural & 1,178 & $28.5 \%$ & $1,171.10$ & 1.005 & $66.7 \%$ \\
\hline 26043 & $\mathrm{MI}$ & Dickinson & rural & 751 & $28.5 \%$ & 761.40 & 0.986 & $65.5 \%$ \\
\hline 26045 & $\mathrm{MI}$ & Eaton & urban & 1,664 & $28.7 \%$ & 575.17 & 2.894 & $57.8 \%$ \\
\hline 26047 & $\mathrm{MI}$ & Emmet & rural & 1,040 & $28.6 \%$ & 467.49 & 2.224 & $62.4 \%$ \\
\hline 26049 & $\mathrm{MI}$ & Genesee & urban & 5,614 & $28.5 \%$ & 636.98 & 8.813 & $48.5 \%$ \\
\hline 26051 & $\mathrm{MI}$ & Gladwin & rural & 790 & $28.7 \%$ & 501.78 & 1.575 & $62.2 \%$ \\
\hline 26053 & $\mathrm{MI}$ & Gogebic & rural & 521 & $28.5 \%$ & $1,101.85$ & 0.473 & $65.1 \%$ \\
\hline 26055 & $\mathrm{MI}$ & Grand Traverse & rural & 2,257 & $28.6 \%$ & 464.33 & 4.860 & $54.4 \%$ \\
\hline 26057 & $\mathrm{MI}$ & Gratiot & rural & 889 & $28.7 \%$ & 568.46 & 1.564 & $65.2 \%$ \\
\hline 26059 & $\mathrm{MI}$ & Hillsdale & rural & 970 & $28.6 \%$ & 598.13 & 1.621 & $63.1 \%$ \\
\hline 26061 & $\mathrm{MI}$ & Houghton & rural & 758 & $28.7 \%$ & $1,009.10$ & 0.751 & $63.9 \%$ \\
\hline 26063 & $\mathrm{MI}$ & Huron & rural & 1,004 & $28.7 \%$ & 835.71 & 1.201 & $70.3 \%$ \\
\hline 26065 & $\mathrm{MI}$ & Ingham & urban & 5,230 & $28.5 \%$ & 556.12 & 9.404 & $58.8 \%$ \\
\hline
\end{tabular}




\begin{tabular}{|c|c|c|c|c|c|c|c|c|}
\hline 26071 & $\mathrm{Ml}$ & Iron & rural & 436 & $28.2 \%$ & $1,166.15$ & 0.374 & $69.8 \%$ \\
\hline 26073 & $\mathrm{Ml}$ & Isabella & rural & 949 & $28.6 \%$ & 572.68 & 1.658 & $58.0 \%$ \\
\hline 26075 & $\mathrm{Ml}$ & Jackson & urban & 3,305 & $28.5 \%$ & 701.67 & 4.710 & $63.1 \%$ \\
\hline 26077 & $\mathrm{MI}$ & Kalamazoo & urban & 3,728 & $28.5 \%$ & 561.66 & 6.637 & $49.0 \%$ \\
\hline 26079 & $\mathrm{Ml}$ & Kalkaska & rural & 336 & $28.6 \%$ & 559.86 & 0.601 & $63.8 \%$ \\
\hline 26081 & $\mathrm{Ml}$ & Kent & urban & 6,248 & $28.3 \%$ & 846.95 & 7.377 & $34.7 \%$ \\
\hline 26083 & $\mathrm{Ml}$ & Keweenaw & rural & 132 & $28.6 \%$ & 540.11 & 0.245 & $65.0 \%$ \\
\hline 26085 & $\mathrm{MI}$ & Lake & rural & 307 & $28.6 \%$ & 567.37 & 0.542 & $64.3 \%$ \\
\hline 26087 & $\mathrm{MI}$ & Lapeer & urban & 1,402 & $28.7 \%$ & 643.01 & 2.180 & $54.0 \%$ \\
\hline 26089 & $\mathrm{MI}$ & Leelanau & rural & 586 & $28.5 \%$ & 347.17 & 1.687 & $56.2 \%$ \\
\hline 26093 & $\mathrm{MI}$ & Livingston & urban & 2,614 & $28.7 \%$ & 565.25 & 4.624 & $52.6 \%$ \\
\hline 26095 & $\mathrm{MI}$ & Luce & rural & 176 & $28.7 \%$ & 899.08 & 0.196 & $71.7 \%$ \\
\hline 26097 & $\mathrm{MI}$ & Mackinac & rural & 329 & $28.5 \%$ & $1,021.57$ & 0.322 & $63.5 \%$ \\
\hline 26099 & $\mathrm{MI}$ & Macomb & urban & 12,436 & $28.7 \%$ & 479.22 & 25.951 & $52.4 \%$ \\
\hline 26101 & $\mathrm{MI}$ & Manistee & rural & 707 & $28.6 \%$ & 542.15 & 1.304 & $61.9 \%$ \\
\hline 26103 & $\mathrm{MI}$ & Marquette & rural & 1,581 & $28.4 \%$ & $1,808.40$ & 0.874 & $64.1 \%$ \\
\hline 26105 & $\mathrm{MI}$ & Mason & rural & 830 & $28.7 \%$ & 495.07 & 1.677 & $63.4 \%$ \\
\hline 26107 & $\mathrm{MI}$ & Mecosta & rural & 830 & $28.5 \%$ & 555.07 & 1.496 & $53.2 \%$ \\
\hline 26109 & $\mathrm{MI}$ & Menominee & rural & 605 & $28.6 \%$ & $1,044.08$ & 0.580 & $57.2 \%$ \\
\hline 26115 & $\mathrm{MI}$ & Monroe & urban & 2,230 & $28.5 \%$ & 549.39 & 4.060 & $52.8 \%$ \\
\hline 26117 & $\mathrm{MI}$ & Montcalm & urban & 1,203 & $28.5 \%$ & 705.40 & 1.705 & $53.7 \%$ \\
\hline 26119 & $\mathrm{MI}$ & Montmorency & rural & 415 & $28.8 \%$ & 546.66 & 0.758 & $66.2 \%$ \\
\hline 26121 & $\mathrm{MI}$ & Muskegon & urban & 2,544 & $28.3 \%$ & 499.25 & 5.097 & $45.7 \%$ \\
\hline 26123 & $\mathrm{MI}$ & Newaygo & rural & 668 & $28.4 \%$ & 813.20 & 0.822 & $40.8 \%$ \\
\hline 26125 & $\mathrm{MI}$ & Oakland & urban & 18,200 & $28.6 \%$ & 867.66 & 20.975 & $51.6 \%$ \\
\hline 26127 & $\mathrm{MI}$ & Oceana & rural & 604 & $28.7 \%$ & 512.07 & 1.180 & $53.8 \%$ \\
\hline 26129 & $\mathrm{MI}$ & Ogemaw & rural & 641 & $28.6 \%$ & 563.49 & 1.137 & $69.5 \%$ \\
\hline 26131 & $\mathrm{MI}$ & Ontonagon & rural & 306 & $28.5 \%$ & $1,311.22$ & 0.234 & $71.4 \%$ \\
\hline 26133 & $\mathrm{MI}$ & Osceola & rural & 623 & $28.7 \%$ & 566.39 & 1.100 & $59.5 \%$ \\
\hline 26135 & $\mathrm{MI}$ & Oscoda & rural & 228 & $28.6 \%$ & 565.73 & 0.403 & $70.7 \%$ \\
\hline 26137 & $\mathrm{MI}$ & Otsego & rural & 623 & $28.7 \%$ & 514.97 & 1.209 & $62.0 \%$ \\
\hline 26139 & $\mathrm{MI}$ & Ottawa & urban & 2,771 & $28.4 \%$ & 563.47 & 4.917 & $29.8 \%$ \\
\hline 26141 & $\mathrm{MI}$ & Presque Isle & rural & 510 & $28.5 \%$ & 658.72 & 0.773 & $68.0 \%$ \\
\hline 26143 & $\mathrm{MI}$ & Roscommon & rural & 945 & $28.7 \%$ & 519.64 & 1.818 & $64.0 \%$ \\
\hline 26145 & $\mathrm{MI}$ & Saginaw & urban & 3,558 & $28.5 \%$ & 800.11 & 4.447 & $55.2 \%$ \\
\hline 26151 & $\mathrm{MI}$ & Sanilac & rural & 945 & $28.6 \%$ & 962.57 & 0.982 & $64.8 \%$ \\
\hline 26153 & $\mathrm{MI}$ & Schoolcraft & rural & 306 & $28.4 \%$ & $1,171.36$ & 0.261 & $73.2 \%$ \\
\hline 26157 & $\mathrm{MI}$ & Tuscola & rural & 1,206 & $28.6 \%$ & 803.13 & 1.502 & $61.5 \%$ \\
\hline 26159 & $\mathrm{MI}$ & Van Buren & urban & 1,632 & $28.5 \%$ & 607.47 & 2.686 & $56.5 \%$ \\
\hline 26161 & $\mathrm{MI}$ & Washtenaw & urban & 5,308 & $28.6 \%$ & 705.97 & 7.519 & $56.6 \%$ \\
\hline 26163 & $\mathrm{MI}$ & Wayne & urban & 20,256 & $28.4 \%$ & 612.08 & 33.094 & $49.5 \%$ \\
\hline 26165 & $\mathrm{MI}$ & Wexford & rural & 800 & $28.5 \%$ & 565.00 & 1.416 & $62.4 \%$ \\
\hline 27001 & $\mathrm{MN}$ & Aitkin & rural & 362 & $28.6 \%$ & $1,821.66$ & 0.199 & $34.9 \%$ \\
\hline 27003 & MN & Anoka & urban & 2,167 & $28.4 \%$ & 423.01 & 5.122 & $24.5 \%$ \\
\hline 27005 & MN & Becker & rural & 485 & $28.3 \%$ & $1,315.20$ & 0.369 & $34.3 \%$ \\
\hline 27007 & $\mathrm{MN}$ & Beltrami & rural & 615 & $28.5 \%$ & $2,504.94$ & 0.246 & $41.0 \%$ \\
\hline 27009 & MN & Benton & urban & 240 & $28.2 \%$ & 408.30 & 0.588 & $26.7 \%$ \\
\hline 27011 & $\mathrm{MN}$ & Big Stone & rural & 122 & $28.4 \%$ & 499.02 & 0.244 & $44.6 \%$ \\
\hline 27013 & $\mathrm{MN}$ & Blue Earth & urban & 792 & $28.4 \%$ & 747.84 & 1.059 & $37.2 \%$ \\
\hline 27015 & $\mathrm{MN}$ & Brown & rural & 537 & $28.4 \%$ & 611.09 & 0.879 & $46.1 \%$ \\
\hline 27017 & $\mathrm{MN}$ & Carlton & urban & 511 & $28.5 \%$ & 861.38 & 0.593 & $38.1 \%$ \\
\hline 27019 & $\mathrm{MN}$ & Carver & urban & 576 & $28.4 \%$ & 354.33 & 1.625 & $26.5 \%$ \\
\hline 27021 & $\mathrm{MN}$ & Cass & rural & 523 & $28.6 \%$ & $2,021.54$ & 0.259 & $37.2 \%$ \\
\hline 27023 & $\mathrm{MN}$ & Chippewa & rural & 169 & $28.5 \%$ & 581.12 & 0.292 & $35.0 \%$ \\
\hline 27025 & $\mathrm{MN}$ & Chisago & urban & 516 & $28.2 \%$ & 414.86 & 1.244 & $26.3 \%$ \\
\hline 27027 & MN & Clay & urban & 680 & $28.3 \%$ & $1,045.37$ & 0.651 & $40.0 \%$ \\
\hline 27029 & $\mathrm{MN}$ & Clearwater & rural & 145 & $28.5 \%$ & 998.94 & 0.145 & $37.2 \%$ \\
\hline 27031 & $\mathrm{MN}$ & Cook & rural & 132 & $28.4 \%$ & $1,452.28$ & 0.091 & $41.2 \%$ \\
\hline 27033 & $\mathrm{MN}$ & Cottonwood & rural & 180 & $28.1 \%$ & 638.61 & 0.281 & $45.9 \%$ \\
\hline 27035 & $\mathrm{MN}$ & Crow Wing & rural & 1,105 & $28.3 \%$ & 999.09 & 1.106 & $34.7 \%$ \\
\hline 27037 & $\mathrm{MN}$ & Dakota & urban & 2,507 & $28.5 \%$ & 562.17 & 4.459 & $28.9 \%$ \\
\hline 27039 & $\mathrm{MN}$ & Dodge & urban & 232 & $28.3 \%$ & 439.28 & 0.529 & $39.3 \%$ \\
\hline 27041 & $\mathrm{MN}$ & Douglas & rural & 511 & $28.3 \%$ & 637.30 & 0.801 & $26.9 \%$ \\
\hline 27043 & $\mathrm{MN}$ & Faribault & rural & 250 & $28.1 \%$ & 712.48 & 0.350 & $36.0 \%$ \\
\hline 27045 & $\mathrm{MN}$ & Fillmore & urban & 420 & $28.1 \%$ & 861.30 & 0.488 & $43.5 \%$ \\
\hline 27047 & $\mathrm{MN}$ & Freeborn & rural & 480 & $28.3 \%$ & 707.09 & 0.678 & $34.7 \%$ \\
\hline 27049 & $\mathrm{MN}$ & Goodhue & rural & 632 & $28.3 \%$ & 756.84 & 0.835 & $33.8 \%$ \\
\hline 27051 & $\mathrm{MN}$ & Grant & rural & 125 & $28.2 \%$ & 548.16 & 0.227 & $41.4 \%$ \\
\hline 27053 & $\mathrm{MN}$ & Hennepin & urban & 10,796 & $28.3 \%$ & 553.59 & 19.502 & $28.9 \%$ \\
\hline 27055 & $\mathrm{MN}$ & Houston & urban & 381 & $28.4 \%$ & 552.06 & 0.690 & $44.5 \%$ \\
\hline 27057 & $\mathrm{MN}$ & Hubbard & rural & 379 & $28.7 \%$ & 925.67 & 0.410 & $38.2 \%$ \\
\hline 27059 & $\mathrm{MN}$ & Isanti & urban & 333 & $28.5 \%$ & 435.79 & 0.765 & $29.3 \%$ \\
\hline 27061 & $\mathrm{MN}$ & Itasca & rural & 877 & $28.5 \%$ & $2,667.72$ & 0.329 & $42.8 \%$ \\
\hline 27063 & $\mathrm{MN}$ & Jackson & rural & 271 & $28.2 \%$ & 702.98 & 0.385 & $54.4 \%$ \\
\hline
\end{tabular}




\begin{tabular}{|c|c|c|c|c|c|c|c|c|}
\hline 27071 & $\mathrm{MN}$ & Koochiching & rural & 254 & $28.2 \%$ & $3,104.07$ & 0.082 & $39.1 \%$ \\
\hline 27073 & $\mathrm{MN}$ & Lac qui Parle & rural & 111 & $28.2 \%$ & 765.02 & 0.145 & $37.1 \%$ \\
\hline 27075 & $\mathrm{MN}$ & Lake & rural & 230 & $28.7 \%$ & $2,109.29$ & 0.109 & $44.0 \%$ \\
\hline 27079 & $\mathrm{MN}$ & Le Sueur & urban & 389 & $28.3 \%$ & 448.76 & 0.866 & $31.9 \%$ \\
\hline 27081 & $\mathrm{MN}$ & Lincoln & rural & 153 & $28.3 \%$ & 536.76 & 0.284 & $55.6 \%$ \\
\hline 27083 & $\mathrm{MN}$ & Lyon & rural & 491 & $28.4 \%$ & 714.56 & 0.688 & $51.4 \%$ \\
\hline 27089 & $\mathrm{MN}$ & Marshall & rural & 159 & $28.1 \%$ & $1,775.07$ & 0.089 & $41.6 \%$ \\
\hline 27091 & $\mathrm{MN}$ & Martin & rural & 382 & $28.2 \%$ & 712.35 & 0.536 & $40.0 \%$ \\
\hline 27085 & $\mathrm{MN}$ & McLeod & rural & 499 & $28.4 \%$ & 491.47 & 1.016 & $29.9 \%$ \\
\hline 27093 & $\mathrm{MN}$ & Meeker & rural & 226 & $28.2 \%$ & 608.18 & 0.372 & $25.0 \%$ \\
\hline 27097 & $\mathrm{MN}$ & Morrison & rural & 395 & $28.1 \%$ & $1,125.06$ & 0.351 & $29.3 \%$ \\
\hline 27099 & $\mathrm{MN}$ & Mower & rural & 796 & $28.2 \%$ & 711.33 & 1.119 & $50.6 \%$ \\
\hline 27101 & $\mathrm{MN}$ & Murray & rural & 230 & $28.2 \%$ & 704.70 & 0.326 & $59.0 \%$ \\
\hline 27103 & $\mathrm{MN}$ & Nicollet & urban & 327 & $28.5 \%$ & 448.49 & 0.730 & $34.1 \%$ \\
\hline 27105 & $\mathrm{MN}$ & Nobles & rural & 412 & $28.4 \%$ & 715.11 & 0.577 & $60.6 \%$ \\
\hline 27107 & $\mathrm{MN}$ & Norman & rural & 132 & $28.4 \%$ & 872.79 & 0.151 & $47.0 \%$ \\
\hline 27109 & $\mathrm{MN}$ & Olmsted & urban & 2,328 & $28.6 \%$ & 653.35 & 3.563 & $49.1 \%$ \\
\hline 27111 & $\mathrm{MN}$ & Otter Tail & rural & 926 & $28.2 \%$ & 1972.07 & 0.470 & $32.9 \%$ \\
\hline 27113 & $\mathrm{MN}$ & Pennington & rural & 169 & $28.5 \%$ & 616.57 & 0.275 & $33.1 \%$ \\
\hline 27119 & $\mathrm{MN}$ & Polk & urban & 462 & $28.1 \%$ & $1,971.13$ & 0.234 & $40.3 \%$ \\
\hline 27121 & $\mathrm{MN}$ & Pope & rural & 200 & $28.7 \%$ & 669.71 & 0.298 & $36.5 \%$ \\
\hline 27123 & $\mathrm{MN}$ & Ramsey & urban & 6,181 & $28.4 \%$ & 152.21 & 40.610 & $27.5 \%$ \\
\hline 27127 & $\mathrm{MN}$ & Redwood & rural & 294 & $28.5 \%$ & 878.57 & 0.334 & $41.6 \%$ \\
\hline 27129 & $\mathrm{MN}$ & Renville & rural & 280 & $28.5 \%$ & 982.91 & 0.285 & $46.4 \%$ \\
\hline 27131 & $\mathrm{MN}$ & Rice & rural & 673 & $28.4 \%$ & 495.68 & 1.357 & $30.8 \%$ \\
\hline 27133 & $\mathrm{MN}$ & Rock & rural & 212 & $28.1 \%$ & 482.45 & 0.439 & $49.6 \%$ \\
\hline 27135 & $\mathrm{MN}$ & Roseau & rural & 134 & $27.9 \%$ & $1,671.60$ & 0.080 & $24.8 \%$ \\
\hline 27139 & $\mathrm{MN}$ & Scott & urban & 752 & $28.6 \%$ & 356.48 & 2.110 & $27.8 \%$ \\
\hline 27141 & $\mathrm{MN}$ & Sherburne & urban & 464 & $28.5 \%$ & 432.92 & 1.071 & $27.0 \%$ \\
\hline 27143 & $\mathrm{MN}$ & Sibley & urban & 188 & $28.7 \%$ & 588.78 & 0.319 & $35.0 \%$ \\
\hline 27137 & $\mathrm{MN}$ & St. Louis & urban & 2,940 & $28.3 \%$ & 6247.4 & 0.471 & $37.7 \%$ \\
\hline 27145 & $\mathrm{MN}$ & Stearns & urban & 1,807 & $28.4 \%$ & $1,343.13$ & 1.345 & $31.2 \%$ \\
\hline 27147 & $\mathrm{MN}$ & Steele & rural & 401 & $28.2 \%$ & 429.65 & 0.933 & $30.3 \%$ \\
\hline 27149 & $\mathrm{MN}$ & Stevens & rural & 167 & $28.4 \%$ & 563.60 & 0.296 & $49.0 \%$ \\
\hline 27151 & $\mathrm{MN}$ & Swift & rural & 196 & $28.2 \%$ & 742.08 & 0.264 & $44.8 \%$ \\
\hline 27153 & $\mathrm{MN}$ & Todd & rural & 231 & $28.0 \%$ & 944.98 & 0.245 & $25.1 \%$ \\
\hline 27157 & $\mathrm{MN}$ & Wabasha & urban & 385 & $28.6 \%$ & 522.98 & 0.736 & $36.0 \%$ \\
\hline 27165 & $\mathrm{MN}$ & Watonwan & rural & 222 & $28.4 \%$ & 434.95 & 0.509 & $46.6 \%$ \\
\hline 27167 & $\mathrm{MN}$ & Wilkin & rural & 106 & $28.4 \%$ & 750.96 & 0.141 & $41.0 \%$ \\
\hline 27169 & $\mathrm{MN}$ & Winona & rural & 733 & $28.5 \%$ & 626.21 & 1.170 & $44.2 \%$ \\
\hline 27171 & $\mathrm{MN}$ & Wright & urban & 916 & $28.3 \%$ & 661.46 & 1.385 & $26.3 \%$ \\
\hline 27173 & $\mathrm{MN}$ & Yellow Medicine & rural & 248 & $28.4 \%$ & 759.1 & 0.326 & $51.3 \%$ \\
\hline 29001 & $\mathrm{MO}$ & Adair & rural & 595 & $28.6 \%$ & 567.32 & 1.048 & $87.3 \%$ \\
\hline 29003 & $\mathrm{MO}$ & Andrew & urban & 346 & $28.6 \%$ & 432.70 & 0.799 & $85.6 \%$ \\
\hline 29005 & MO & Atchison & rural & 232 & $28.6 \%$ & 547.30 & 0.423 & $90.4 \%$ \\
\hline 29007 & MO & Audrain & rural & 617 & $28.4 \%$ & 692.23 & 0.891 & $77.3 \%$ \\
\hline 29009 & MO & Barry & rural & 895 & $28.5 \%$ & 778.25 & 1.150 & $56.2 \%$ \\
\hline 29011 & $\mathrm{MO}$ & Barton & rural & 289 & $28.5 \%$ & 591.92 & 0.488 & $73.2 \%$ \\
\hline 29013 & $\mathrm{MO}$ & Bates & urban & 450 & $28.7 \%$ & 836.69 & 0.537 & $74.9 \%$ \\
\hline 29015 & $\mathrm{MO}$ & Benton & rural & 765 & $28.7 \%$ & 704.06 & 1.087 & $70.5 \%$ \\
\hline 29017 & $\mathrm{MO}$ & Bollinger & urban & 299 & $28.4 \%$ & 617.91 & 0.483 & $78.7 \%$ \\
\hline 29019 & $\mathrm{MO}$ & Boone & urban & 3,010 & $28.6 \%$ & 685.41 & 4.392 & $73.8 \%$ \\
\hline 29021 & $\mathrm{MO}$ & Buchanan & urban & 2,211 & $28.5 \%$ & 408.03 & 5.418 & $83.2 \%$ \\
\hline 29023 & $\mathrm{MO}$ & Butler & rural & 1,076 & $28.7 \%$ & 694.67 & 1.549 & $81.1 \%$ \\
\hline 29025 & $\mathrm{MO}$ & Caldwell & urban & 283 & $28.4 \%$ & 426.39 & 0.664 & $79.0 \%$ \\
\hline 29027 & $\mathrm{MO}$ & Callaway & urban & 910 & $28.4 \%$ & 834.57 & 1.090 & $74.8 \%$ \\
\hline 29029 & MO & Camden & rural & 1,454 & $28.7 \%$ & 655.92 & 2.217 & $78.3 \%$ \\
\hline 29031 & $\mathrm{MO}$ & Cape Girardeau & urban & 1,987 & $28.7 \%$ & 578.53 & 3.434 & $84.3 \%$ \\
\hline 29033 & $\mathrm{MO}$ & Carroll & rural & 308 & $28.6 \%$ & 694.62 & 0.444 & $81.0 \%$ \\
\hline 29035 & $\mathrm{MO}$ & Carter & rural & 192 & $28.3 \%$ & 507.36 & 0.378 & $79.6 \%$ \\
\hline 29037 & $\mathrm{MO}$ & Cass & urban & 1,444 & $28.5 \%$ & 696.84 & 2.073 & $50.3 \%$ \\
\hline 29039 & MO & Cedar & rural & 397 & $28.6 \%$ & 474.48 & 0.837 & $59.4 \%$ \\
\hline 29041 & $\mathrm{MO}$ & Chariton & rural & 242 & $28.5 \%$ & 751.18 & 0.322 & $85.4 \%$ \\
\hline 29043 & $\mathrm{MO}$ & Christian & urban & 1,275 & $28.6 \%$ & 562.65 & 2.266 & $46.5 \%$ \\
\hline 29045 & $\mathrm{MO}$ & Clark & rural & 191 & $28.7 \%$ & 504.69 & 0.378 & $85.9 \%$ \\
\hline 29047 & $\mathrm{MO}$ & Clay & urban & 4,429 & $28.7 \%$ & 397.30 & 11.147 & $64.7 \%$ \\
\hline 29049 & MO & Clinton & urban & 603 & $28.7 \%$ & 418.96 & 1.438 & $80.0 \%$ \\
\hline 29051 & $\mathrm{MO}$ & Cole & urban & 1,871 & $28.6 \%$ & 393.75 & 4.751 & $79.6 \%$ \\
\hline 29053 & $\mathrm{MO}$ & Cooper & rural & 433 & $28.6 \%$ & 564.77 & 0.766 & $75.0 \%$ \\
\hline 29055 & $\mathrm{MO}$ & Crawford & rural & 439 & $28.3 \%$ & 742.52 & 0.592 & $63.5 \%$ \\
\hline 29057 & $\mathrm{MO}$ & Dade & rural & 183 & $28.4 \%$ & 490.01 & 0.373 & $52.8 \%$ \\
\hline 29059 & $\mathrm{MO}$ & Dallas & urban & 265 & $28.4 \%$ & 540.77 & 0.490 & $44.1 \%$ \\
\hline 29061 & $\mathrm{MO}$ & Daviess & rural & 242 & $28.4 \%$ & 563.24 & 0.430 & $84.2 \%$ \\
\hline 29063 & $\mathrm{MO}$ & DeKalb & urban & 236 & $28.6 \%$ & 421.36 & 0.561 & $84.8 \%$ \\
\hline
\end{tabular}




\begin{tabular}{|c|c|c|c|c|c|c|c|c|}
\hline 29069 & $\mathrm{MO}$ & Dunklin & rural & 807 & $28.6 \%$ & 541.07 & 1.491 & $82.0 \%$ \\
\hline 29071 & $\mathrm{MO}$ & Franklin & urban & 1,591 & $28.6 \%$ & 922.68 & 1.724 & $41.1 \%$ \\
\hline 29073 & $\mathrm{MO}$ & Gasconade & rural & 455 & $28.6 \%$ & 517.80 & 0.878 & $66.6 \%$ \\
\hline 29075 & MO & Gentry & rural & 220 & $28.5 \%$ & 491.42 & 0.447 & $89.1 \%$ \\
\hline 29077 & MO & Greene & urban & 4,725 & $28.6 \%$ & 675.30 & 6.997 & $46.8 \%$ \\
\hline 29079 & $\mathrm{MO}$ & Grundy & rural & 378 & $28.7 \%$ & 435.28 & 0.869 & $90.0 \%$ \\
\hline 29081 & $\mathrm{MO}$ & Harrison & rural & 334 & $28.4 \%$ & 722.50 & 0.463 & $89.6 \%$ \\
\hline 29083 & $\mathrm{MO}$ & Henry & rural & 713 & $28.6 \%$ & 696.95 & 1.023 & $70.5 \%$ \\
\hline 29085 & MO & Hickory & rural & 281 & $28.7 \%$ & 399.09 & 0.703 & $56.5 \%$ \\
\hline 29087 & MO & Holt & rural & 172 & $28.1 \%$ & 462.69 & 0.372 & $86.0 \%$ \\
\hline 29091 & $\mathrm{MO}$ & Howell & rural & 1,203 & $28.6 \%$ & 927.25 & 1.297 & $71.8 \%$ \\
\hline 29093 & $\mathrm{MO}$ & Iron & rural & 317 & $28.6 \%$ & 550.26 & 0.575 & $82.2 \%$ \\
\hline 29095 & $\mathrm{MO}$ & Jackson & urban & 10,180 & $28.5 \%$ & 604.46 & 16.841 & $48.9 \%$ \\
\hline 29097 & $\mathrm{MO}$ & Jasper & urban & 2,733 & $28.6 \%$ & 638.49 & 4.281 & $67.0 \%$ \\
\hline 29099 & MO & Jefferson & urban & 2,674 & $28.6 \%$ & 656.63 & 4.072 & $43.4 \%$ \\
\hline 29101 & MO & Johnson & rural & 876 & $28.6 \%$ & 829.28 & 1.056 & $72.6 \%$ \\
\hline 29103 & $\mathrm{MO}$ & Knox & rural & 143 & $28.3 \%$ & 504.01 & 0.284 & $89.7 \%$ \\
\hline 29105 & $\mathrm{MO}$ & Laclede & rural & 657 & $28.5 \%$ & 764.72 & 0.859 & $45.8 \%$ \\
\hline 29107 & $\mathrm{MO}$ & Lafayette & urban & 793 & $28.6 \%$ & 628.43 & 1.263 & $65.6 \%$ \\
\hline 29113 & $\mathrm{MO}$ & Lincoln & urban & 720 & $28.6 \%$ & 626.56 & 1.149 & $54.7 \%$ \\
\hline 29115 & $\mathrm{MO}$ & Linn & rural & 465 & $28.5 \%$ & 615.56 & 0.756 & $85.2 \%$ \\
\hline 29117 & $\mathrm{MO}$ & Livingston & rural & 432 & $28.6 \%$ & 532.33 & 0.812 & $83.8 \%$ \\
\hline 29121 & $\mathrm{MO}$ & Macon & rural & 538 & $28.5 \%$ & 801.23 & 0.671 & $86.3 \%$ \\
\hline 29123 & $\mathrm{MO}$ & Madison & rural & 345 & $28.3 \%$ & 494.39 & 0.697 & $79.2 \%$ \\
\hline 29125 & $\mathrm{MO}$ & Maries & rural & 195 & $28.9 \%$ & 526.98 & 0.371 & $80.1 \%$ \\
\hline 29127 & $\mathrm{MO}$ & Marion & rural & 815 & $28.6 \%$ & 436.92 & 1.864 & $80.2 \%$ \\
\hline 29119 & $\mathrm{MO}$ & McDonald & urban & 364 & $28.7 \%$ & 539.48 & 0.676 & $57.3 \%$ \\
\hline 29129 & $\mathrm{MO}$ & Mercer & rural & 116 & $28.3 \%$ & 453.84 & 0.256 & $86.7 \%$ \\
\hline 29131 & $\mathrm{MO}$ & Miller & rural & 791 & $28.5 \%$ & 592.59 & 1.334 & $75.9 \%$ \\
\hline 29133 & $\mathrm{MO}$ & Mississippi & rural & 367 & $28.5 \%$ & 411.58 & 0.891 & $87.8 \%$ \\
\hline 29135 & $\mathrm{MO}$ & Moniteau & urban & 346 & $28.6 \%$ & 415.03 & 0.833 & $79.2 \%$ \\
\hline 29137 & $\mathrm{MO}$ & Monroe & rural & 328 & $28.3 \%$ & 647.65 & 0.506 & $84.5 \%$ \\
\hline 29139 & $\mathrm{MO}$ & Montgomery & rural & 340 & $28.7 \%$ & 536.25 & 0.634 & $74.9 \%$ \\
\hline 29141 & $\mathrm{MO}$ & Morgan & rural & 754 & $28.6 \%$ & 597.63 & 1.261 & $79.0 \%$ \\
\hline 29143 & $\mathrm{MO}$ & New Madrid & rural & 383 & $28.7 \%$ & 674.84 & 0.567 & $81.3 \%$ \\
\hline 29145 & $\mathrm{MO}$ & Newton & urban & 950 & $28.7 \%$ & 624.76 & 1.521 & $67.5 \%$ \\
\hline 29147 & $\mathrm{MO}$ & Nodaway & rural & 593 & $28.7 \%$ & 876.96 & 0.676 & $89.5 \%$ \\
\hline 29155 & MO & Pemiscot & rural & 316 & $28.5 \%$ & 492.54 & 0.642 & $74.2 \%$ \\
\hline 29157 & MO & Perry & rural & 504 & $28.5 \%$ & 474.35 & 1.064 & $83.9 \%$ \\
\hline 29159 & MO & Pettis & rural & 1,069 & $28.6 \%$ & 682.22 & 1.566 & $78.5 \%$ \\
\hline 29161 & MO & Phelps & rural & 1,072 & $28.6 \%$ & 671.78 & 1.595 & $77.3 \%$ \\
\hline 29163 & $\mathrm{MO}$ & Pike & rural & 424 & $28.9 \%$ & 670.44 & 0.632 & $76.7 \%$ \\
\hline 29165 & $\mathrm{MO}$ & Platte & urban & 1,622 & $28.5 \%$ & 420.19 & 3.861 & $64.2 \%$ \\
\hline 29167 & $\mathrm{MO}$ & Polk & urban & 576 & $28.4 \%$ & 635.52 & 0.906 & $46.0 \%$ \\
\hline 29169 & $\mathrm{MO}$ & Pulaski & rural & 740 & $28.5 \%$ & 547.10 & 1.353 & $80.6 \%$ \\
\hline 29171 & MO & Putnam & rural & 187 & $28.4 \%$ & 517.32 & 0.361 & $86.8 \%$ \\
\hline 29173 & $\mathrm{MO}$ & Ralls & rural & 229 & $28.4 \%$ & 469.78 & 0.487 & $80.2 \%$ \\
\hline 29175 & $\mathrm{MO}$ & Randolph & rural & 597 & $28.4 \%$ & 482.68 & 1.237 & $76.1 \%$ \\
\hline 29177 & $\mathrm{MO}$ & Ray & urban & 499 & $28.7 \%$ & 568.81 & 0.877 & $77.4 \%$ \\
\hline 29179 & $\mathrm{MO}$ & Reynolds & rural & 176 & $28.6 \%$ & 808.48 & 0.218 & $80.0 \%$ \\
\hline 29181 & $\mathrm{MO}$ & Ripley & rural & 360 & $28.7 \%$ & 629.54 & 0.572 & $82.1 \%$ \\
\hline 29195 & $\mathrm{MO}$ & Saline & rural & 507 & $28.7 \%$ & 755.50 & 0.671 & $73.0 \%$ \\
\hline 29197 & $\mathrm{MO}$ & Schuyler & rural & 169 & $28.9 \%$ & 307.30 & 0.549 & $82.5 \%$ \\
\hline 29199 & $\mathrm{MO}$ & Scotland & rural & 139 & $28.6 \%$ & 436.67 & 0.318 & $89.2 \%$ \\
\hline 29201 & $\mathrm{MO}$ & Scott & rural & 1,116 & $28.6 \%$ & 419.99 & 2.657 & $82.4 \%$ \\
\hline 29203 & $\mathrm{MO}$ & Shannon & rural & 203 & $28.8 \%$ & $1,003.82$ & 0.203 & $75.5 \%$ \\
\hline 29205 & MO & Shelby & rural & 243 & $28.7 \%$ & 500.86 & 0.485 & $87.9 \%$ \\
\hline 29183 & $\mathrm{MO}$ & St. Charles & urban & 5,487 & $28.7 \%$ & 560.44 & 9.790 & $46.4 \%$ \\
\hline 29185 & $\mathrm{MO}$ & St. Clair & rural & 292 & $28.8 \%$ & 669.98 & 0.436 & $69.3 \%$ \\
\hline 29187 & $\mathrm{MO}$ & St. Francois & rural & 1,422 & $28.7 \%$ & 451.89 & 3.146 & $73.7 \%$ \\
\hline 29189 & $\mathrm{MO}$ & St. Louis & urban & 17,975 & $28.5 \%$ & 507.8 & 35.397 & $48.7 \%$ \\
\hline 29510 & MO & St. Louis City & urban & 3,042 & $28.0 \%$ & 61.91 & 49.143 & $45.3 \%$ \\
\hline 29186 & $\mathrm{MO}$ & Ste. Genevieve & rural & 439 & $28.6 \%$ & 499.15 & 0.879 & $77.3 \%$ \\
\hline 29207 & $\mathrm{MO}$ & Stoddard & rural & 838 & $28.6 \%$ & 823.22 & 1.018 & $77.7 \%$ \\
\hline 29209 & $\mathrm{MO}$ & Stone & rural & 914 & $28.7 \%$ & 464.03 & 1.971 & $54.7 \%$ \\
\hline 29211 & $\mathrm{MO}$ & Sullivan & rural & 197 & $28.5 \%$ & 647.98 & 0.304 & $86.6 \%$ \\
\hline 29213 & MO & Taney & rural & 1,275 & $28.7 \%$ & 632.44 & 2.016 & $54.5 \%$ \\
\hline 29215 & $\mathrm{MO}$ & Texas & rural & 760 & $28.5 \%$ & $1,177.27$ & 0.645 & $81.9 \%$ \\
\hline 29217 & $\mathrm{MO}$ & Vernon & rural & 533 & $28.7 \%$ & 826.40 & 0.645 & $74.3 \%$ \\
\hline 29219 & $\mathrm{MO}$ & Warren & urban & 476 & $28.5 \%$ & 428.60 & 1.111 & $45.3 \%$ \\
\hline 29221 & $\mathrm{MO}$ & Washington & rural & 473 & $28.6 \%$ & 759.91 & 0.623 & $72.3 \%$ \\
\hline 29223 & $\mathrm{MO}$ & Wayne & rural & 479 & $28.7 \%$ & 759.18 & 0.631 & $77.6 \%$ \\
\hline 29225 & $\mathrm{MO}$ & Webster & urban & 611 & $28.3 \%$ & 592.56 & 1.030 & $40.3 \%$ \\
\hline 29229 & $\mathrm{MO}$ & Wright & rural & 444 & $28.4 \%$ & 681.77 & 0.651 & $53.1 \%$ \\
\hline
\end{tabular}




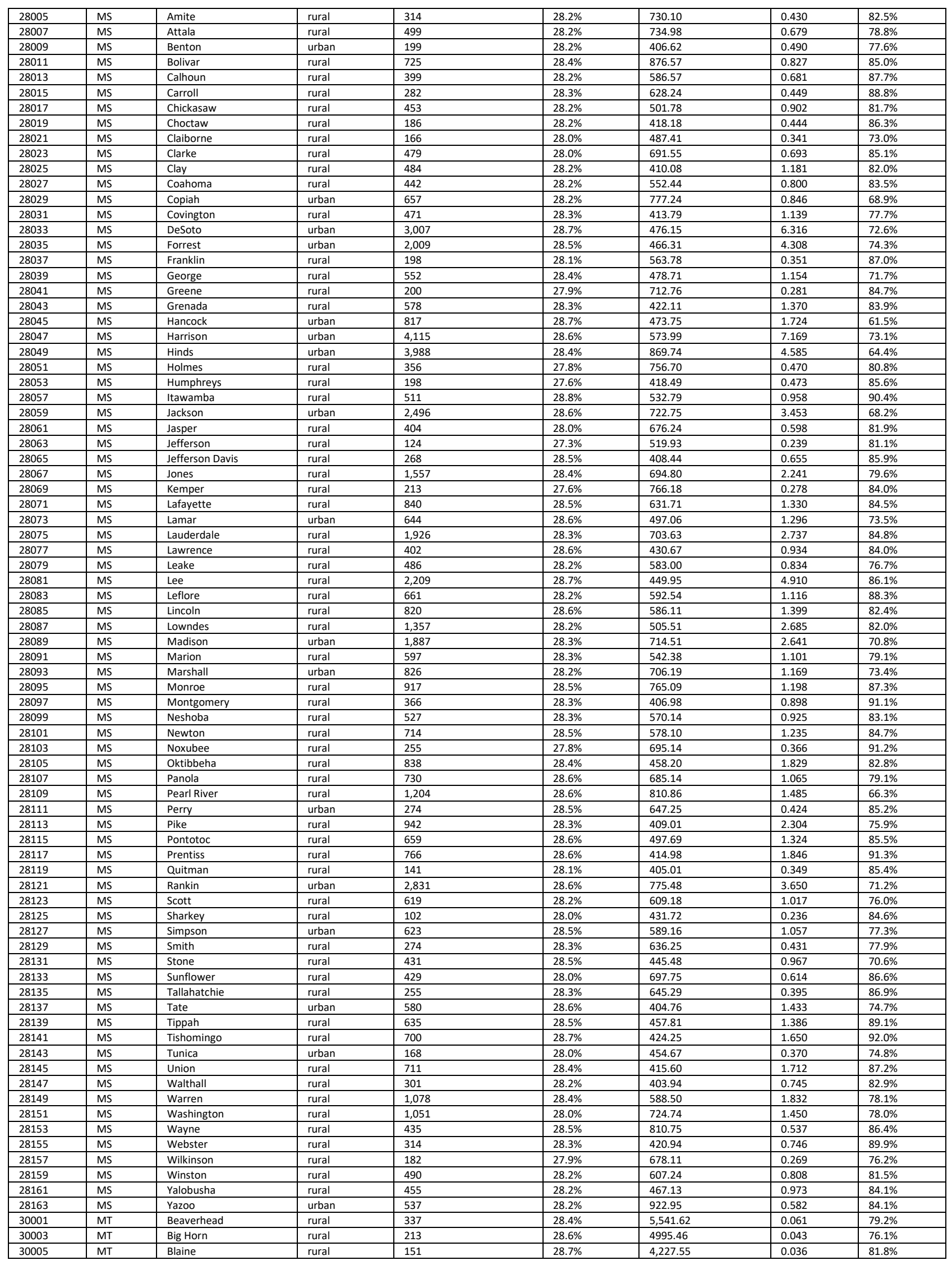




\begin{tabular}{|c|c|c|c|c|c|c|c|c|}
\hline 30007 & MT & Broadwater & rural & 218 & $28.6 \%$ & $1,192.54$ & 0.183 & $70.5 \%$ \\
\hline 30009 & MT & Carbon & urban & 357 & $28.2 \%$ & $2,048.79$ & 0.174 & $70.7 \%$ \\
\hline 30013 & MT & Cascade & urban & 2,063 & $28.4 \%$ & $2,698.16$ & 0.764 & $66.5 \%$ \\
\hline 30015 & MT & Chouteau & rural & 162 & $28.2 \%$ & $3,972.49$ & 0.041 & $74.3 \%$ \\
\hline 30017 & MT & Custer & rural & 377 & $28.4 \%$ & $3,783.36$ & 0.100 & $83.6 \%$ \\
\hline 30021 & MT & Dawson & rural & 291 & $28.4 \%$ & $2,371.86$ & 0.123 & $92.0 \%$ \\
\hline 30023 & MT & Deer Lodge & rural & 350 & $28.5 \%$ & 736.53 & 0.475 & $81.3 \%$ \\
\hline 30027 & MT & Fergus & rural & 415 & $28.2 \%$ & $4,339.80$ & 0.096 & $71.8 \%$ \\
\hline 30029 & MT & Flathead & rural & 3,002 & $28.5 \%$ & $5,087.66$ & 0.590 & $69.1 \%$ \\
\hline 30031 & MT & Gallatin & rural & 1,964 & $28.4 \%$ & $2,602.69$ & 0.754 & $69.8 \%$ \\
\hline 30039 & MT & Granite & rural & 120 & $28.4 \%$ & $1,727.41$ & 0.070 & $78.6 \%$ \\
\hline 30041 & MT & Hill & rural & 432 & $28.6 \%$ & $2,898.95$ & 0.149 & $85.8 \%$ \\
\hline 30043 & MT & Jefferson & rural & 359 & $28.4 \%$ & $1,656.26$ & 0.216 & $68.6 \%$ \\
\hline 30047 & MT & Lake & rural & 800 & $28.6 \%$ & $1,490.15$ & 0.537 & $72.6 \%$ \\
\hline 30049 & MT & Lewis and Clark & rural & 1,740 & $28.5 \%$ & 3458.83 & 0.503 & $67.9 \%$ \\
\hline 30051 & MT & Liberty & rural & 103 & $28.3 \%$ & $1,430.05$ & 0.072 & $91.3 \%$ \\
\hline 30053 & MT & Lincoln & rural & 689 & $28.4 \%$ & $3,612.92$ & 0.191 & $66.3 \%$ \\
\hline 30057 & MT & Madison & rural & 351 & $28.1 \%$ & $3,587.48$ & 0.098 & $82.6 \%$ \\
\hline 30061 & MT & Mineral & rural & 203 & $28.4 \%$ & $1,219.44$ & 0.167 & $75.3 \%$ \\
\hline 30067 & MT & Park & rural & 525 & $28.5 \%$ & $2,803.06$ & 0.187 & $72.3 \%$ \\
\hline 30071 & MT & Phillips & rural & 165 & $28.5 \%$ & $5,140.04$ & 0.032 & $87.7 \%$ \\
\hline 30073 & MT & Pondera & rural & 195 & $28.4 \%$ & $1,622.86$ & 0.120 & $74.9 \%$ \\
\hline 30077 & MT & Powell & rural & 213 & $28.1 \%$ & $2,326.39$ & 0.091 & $78.5 \%$ \\
\hline 30081 & MT & Ravalli & rural & 1,626 & $28.5 \%$ & $2,390.82$ & 0.680 & $70.0 \%$ \\
\hline 30083 & MT & Richland & rural & 299 & $28.7 \%$ & $2,084.14$ & 0.144 & $92.6 \%$ \\
\hline 30085 & MT & Roosevelt & rural & 235 & $28.6 \%$ & $2,354.79$ & 0.100 & $89.7 \%$ \\
\hline 30087 & MT & Rosebud & rural & 217 & $28.6 \%$ & $5,010.40$ & 0.043 & $76.9 \%$ \\
\hline 30089 & MT & Sanders & rural & 506 & $28.2 \%$ & $2,760.52$ & 0.183 & $73.7 \%$ \\
\hline 30091 & MT & Sheridan & rural & 170 & $28.4 \%$ & $1,677.08$ & 0.101 & $90.2 \%$ \\
\hline 30093 & MT & Silver Bow & rural & 1,013 & $28.5 \%$ & 718.48 & 1.410 & $75.6 \%$ \\
\hline 30095 & MT & Stillwater & rural & 303 & $28.6 \%$ & $1,795.35$ & 0.169 & $68.5 \%$ \\
\hline 30097 & MT & Sweet Grass & rural & 139 & $28.2 \%$ & 1855.2 & 0.075 & $80.1 \%$ \\
\hline 30099 & MT & Teton & rural & 179 & $28.4 \%$ & $2,272.37$ & 0.079 & $60.4 \%$ \\
\hline 30105 & MT & Valley & rural & 328 & $28.4 \%$ & $4,925.82$ & 0.067 & $90.4 \%$ \\
\hline 30111 & MT & Yellowstone & urban & 3,701 & $28.5 \%$ & $2,633.29$ & 1.405 & $68.1 \%$ \\
\hline 37001 & NC & Alamance & urban & 2,052 & $28.5 \%$ & 423.94 & 4.839 & $37.5 \%$ \\
\hline 37003 & $\mathrm{NC}$ & Alexander & urban & 729 & $28.7 \%$ & 259.99 & 2.805 & $59.9 \%$ \\
\hline 37011 & NC & Avery & rural & 578 & $28.7 \%$ & 247.09 & 2.339 & $67.4 \%$ \\
\hline 37013 & $\mathrm{NC}$ & Beaufort & rural & 1,563 & $28.5 \%$ & 827.19 & 1.890 & $77.6 \%$ \\
\hline 37015 & $\mathrm{NC}$ & Bertie & rural & 514 & $27.8 \%$ & 699.27 & 0.734 & $82.3 \%$ \\
\hline 37017 & NC & Bladen & rural & 563 & $28.0 \%$ & 874.33 & 0.644 & $65.3 \%$ \\
\hline 37019 & $\mathrm{NC}$ & Brunswick & urban & 4,986 & $28.7 \%$ & 846.97 & 5.886 & $75.0 \%$ \\
\hline 37021 & $\mathrm{NC}$ & Buncombe & urban & 6,598 & $28.6 \%$ & 656.67 & 10.048 & $65.9 \%$ \\
\hline 37023 & $\mathrm{NC}$ & Burke & urban & 1,627 & $28.6 \%$ & 507.10 & 3.209 & $58.2 \%$ \\
\hline 37025 & $\mathrm{NC}$ & Cabarrus & urban & 2,930 & $28.5 \%$ & 361.75 & 8.099 & $55.9 \%$ \\
\hline 37027 & NC & Caldwell & urban & 1,560 & $28.4 \%$ & 471.57 & 3.309 & $55.6 \%$ \\
\hline 37029 & $\mathrm{NC}$ & Camden & rural & 186 & $28.2 \%$ & 240.56 & 0.773 & $76.5 \%$ \\
\hline 37031 & $\mathrm{NC}$ & Carteret & rural & 1,914 & $28.6 \%$ & 506.25 & 3.780 & $75.3 \%$ \\
\hline 37033 & $\mathrm{NC}$ & Caswell & rural & 336 & $27.9 \%$ & 424.92 & 0.792 & $48.4 \%$ \\
\hline 37035 & $\mathrm{NC}$ & Catawba & urban & 3,329 & $28.6 \%$ & 398.72 & 8.349 & $58.9 \%$ \\
\hline 37037 & NC & Chatham & urban & 1,038 & $28.5 \%$ & 682.19 & 1.522 & $50.6 \%$ \\
\hline 37039 & $\mathrm{NC}$ & Cherokee & rural & 974 & $28.6 \%$ & 455.43 & 2.139 & $68.5 \%$ \\
\hline 37041 & $\mathrm{NC}$ & Chowan & rural & 476 & $28.6 \%$ & 172.47 & 2.759 & $82.2 \%$ \\
\hline 37043 & $\mathrm{NC}$ & Clay & rural & 448 & $28.5 \%$ & 214.75 & 2.087 & $73.5 \%$ \\
\hline 37045 & $\mathrm{NC}$ & Cleveland & rural & 2,277 & $28.5 \%$ & 464.25 & 4.905 & $68.0 \%$ \\
\hline 37047 & $\mathrm{NC}$ & Columbus & rural & 1,180 & $28.2 \%$ & 937.29 & 1.259 & $76.7 \%$ \\
\hline 37049 & $\mathrm{NC}$ & Craven & urban & 2,963 & $28.5 \%$ & 708.96 & 4.179 & $83.5 \%$ \\
\hline 37051 & $\mathrm{NC}$ & Cumberland & urban & 4,382 & $28.4 \%$ & 652.31 & 6.718 & $67.5 \%$ \\
\hline 37053 & $\mathrm{NC}$ & Currituck & urban & 568 & $28.6 \%$ & 261.85 & 2.170 & $79.5 \%$ \\
\hline 37055 & $\mathrm{NC}$ & Dare & rural & 1,185 & $28.7 \%$ & 383.42 & 3.091 & $83.2 \%$ \\
\hline 37057 & $\mathrm{NC}$ & Davidson & urban & 1,723 & $28.4 \%$ & 552.67 & 3.118 & $35.3 \%$ \\
\hline 37059 & NC & Davie & urban & 676 & $28.7 \%$ & 264.11 & 2.559 & $38.9 \%$ \\
\hline 37061 & NC & Duplin & rural & 950 & $28.2 \%$ & 816.22 & 1.164 & $74.2 \%$ \\
\hline 37063 & $\mathrm{NC}$ & Durham & urban & 4,080 & $28.4 \%$ & 285.98 & 14.268 & $58.8 \%$ \\
\hline 37065 & $\mathrm{NC}$ & Edgecombe & urban & 804 & $28.0 \%$ & 505.34 & 1.590 & $73.4 \%$ \\
\hline 37067 & NC & Forsyth & urban & 4,537 & $28.4 \%$ & 408.15 & 11.116 & $34.8 \%$ \\
\hline 37069 & NC & Franklin & urban & 930 & $28.3 \%$ & 491.68 & 1.892 & $60.0 \%$ \\
\hline 37071 & NC & Gaston & urban & 3,504 & $28.6 \%$ & 356.03 & 9.843 & $54.4 \%$ \\
\hline 37073 & NC & Gates & urban & 244 & $28.2 \%$ & 340.44 & 0.718 & $74.4 \%$ \\
\hline 37075 & NC & Graham & rural & 231 & $28.3 \%$ & 292.08 & 0.790 & $70.0 \%$ \\
\hline 37077 & NC & Granville & rural & 833 & $28.2 \%$ & 531.57 & 1.567 & $58.3 \%$ \\
\hline 37079 & NC & Greene & rural & 301 & $28.2 \%$ & 265.93 & 1.131 & $77.8 \%$ \\
\hline 37081 & NC & Guilford & urban & 6,177 & $28.4 \%$ & 645.70 & 9.567 & $38.3 \%$ \\
\hline 37083 & NC & Halifax & rural & 1,236 & $27.9 \%$ & 724.09 & 1.707 & $70.2 \%$ \\
\hline
\end{tabular}




\begin{tabular}{|c|c|c|c|c|c|c|c|c|}
\hline 37089 & $\mathrm{NC}$ & Henderson & urban & 4,182 & $28.8 \%$ & 373.07 & 11.210 & $68.2 \%$ \\
\hline 37091 & $\mathrm{NC}$ & Hertford & rural & 500 & $27.8 \%$ & 353.06 & 1.415 & $79.4 \%$ \\
\hline 37093 & $\mathrm{NC}$ & Hoke & urban & 339 & $28.3 \%$ & 390.74 & 0.868 & $63.1 \%$ \\
\hline 37095 & $\mathrm{NC}$ & Hyde & rural & 111 & $28.7 \%$ & 612.70 & 0.182 & $74.1 \%$ \\
\hline 37097 & $\mathrm{NC}$ & Iredell & urban & 2,853 & $28.5 \%$ & 573.83 & 4.972 & $57.3 \%$ \\
\hline 37099 & $\mathrm{NC}$ & Jackson & rural & 931 & $28.7 \%$ & 490.75 & 1.897 & $67.0 \%$ \\
\hline 37101 & $\mathrm{NC}$ & Johnston & urban & 2,386 & $28.5 \%$ & 791.30 & 3.016 & $66.8 \%$ \\
\hline 37103 & $\mathrm{NC}$ & Jones & urban & 245 & $28.3 \%$ & 470.71 & 0.522 & $77.5 \%$ \\
\hline 37105 & $\mathrm{NC}$ & Lee & rural & 1,441 & $28.6 \%$ & 254.96 & 5.652 & $68.4 \%$ \\
\hline 37107 & $\mathrm{NC}$ & Lenoir & rural & 1,519 & $28.4 \%$ & 400.59 & 3.793 & $78.3 \%$ \\
\hline 37113 & NC & Macon & rural & 1,564 & $28.8 \%$ & 515.56 & 3.034 & $80.4 \%$ \\
\hline 37115 & $\mathrm{NC}$ & Madison & urban & 589 & $28.6 \%$ & 449.57 & 1.310 & $66.4 \%$ \\
\hline 37117 & $\mathrm{NC}$ & Martin & rural & 611 & $28.1 \%$ & 461.22 & 1.326 & $78.5 \%$ \\
\hline 37111 & NC & McDowell & rural & 1,015 & $28.4 \%$ & 440.61 & 2.303 & $60.0 \%$ \\
\hline 37119 & $\mathrm{NC}$ & Mecklenburg & urban & 13,349 & $28.5 \%$ & 523.84 & 25.484 & $57.9 \%$ \\
\hline 37121 & NC & Mitchell & rural & 457 & $28.5 \%$ & 221.42 & 2.065 & $66.0 \%$ \\
\hline 37123 & $\mathrm{NC}$ & Montgomery & rural & 528 & $28.4 \%$ & 491.76 & 1.074 & $65.4 \%$ \\
\hline 37125 & $\mathrm{NC}$ & Moore & rural & 3,055 & $28.6 \%$ & 697.84 & 4.377 & $68.0 \%$ \\
\hline 37127 & $\mathrm{NC}$ & Nash & urban & 2,349 & $28.3 \%$ & 540.41 & 4.347 & $71.5 \%$ \\
\hline 37133 & $\mathrm{NC}$ & Onslow & urban & 2,381 & $28.5 \%$ & 762.74 & 3.121 & $78.9 \%$ \\
\hline 37135 & $\mathrm{NC}$ & Orange & urban & 2,472 & $28.5 \%$ & 397.96 & 6.213 & $55.5 \%$ \\
\hline 37137 & $\mathrm{NC}$ & Pamlico & urban & 454 & $28.3 \%$ & 336.54 & 1.349 & $82.5 \%$ \\
\hline 37139 & $\mathrm{NC}$ & Pasquotank & rural & 892 & $28.3 \%$ & 226.88 & 3.931 & $77.6 \%$ \\
\hline 37141 & NC & Pender & urban & 1,271 & $28.4 \%$ & 869.79 & 1.462 & $74.7 \%$ \\
\hline 37143 & NC & Perquimans & rural & 481 & $28.6 \%$ & 247.09 & 1.948 & $81.2 \%$ \\
\hline 37145 & $\mathrm{NC}$ & Person & urban & 654 & $28.4 \%$ & 392.32 & 1.668 & $54.5 \%$ \\
\hline 37147 & $\mathrm{NC}$ & Pitt & urban & 2,863 & $28.4 \%$ & 651.97 & 4.392 & $73.9 \%$ \\
\hline 37149 & $\mathrm{NC}$ & Polk & rural & 841 & $28.6 \%$ & 237.79 & 3.536 & $73.2 \%$ \\
\hline 37151 & $\mathrm{NC}$ & Randolph & urban & 1,823 & $28.6 \%$ & 782.52 & 2.330 & $36.4 \%$ \\
\hline 37153 & $\mathrm{NC}$ & Richmond & rural & 946 & $28.4 \%$ & 473.82 & 1.997 & $75.1 \%$ \\
\hline 37155 & $\mathrm{NC}$ & Robeson & rural & 2,062 & $28.4 \%$ & 949.22 & 2.172 & $66.4 \%$ \\
\hline 37157 & $\mathrm{NC}$ & Rockingham & urban & 1,281 & $28.3 \%$ & 565.55 & 2.266 & $37.5 \%$ \\
\hline 37159 & $\mathrm{NC}$ & Rowan & urban & 2,050 & $28.4 \%$ & 511.37 & 4.009 & $49.2 \%$ \\
\hline 37161 & $\mathrm{NC}$ & Rutherford & rural & 1,711 & $28.4 \%$ & 564.15 & 3.033 & $71.9 \%$ \\
\hline 37163 & $\mathrm{NC}$ & Sampson & rural & 1,012 & $28.4 \%$ & 944.74 & 1.071 & $69.1 \%$ \\
\hline 37165 & $\mathrm{NC}$ & Scotland & rural & 662 & $28.4 \%$ & 318.84 & 2.075 & $70.0 \%$ \\
\hline 37167 & $\mathrm{NC}$ & Stanly & rural & 1,331 & $28.5 \%$ & 395.09 & 3.369 & $63.5 \%$ \\
\hline 37175 & NC & Transylvania & rural & 1,327 & $28.9 \%$ & 378.53 & 3.506 & $68.9 \%$ \\
\hline 37179 & $\mathrm{NC}$ & Union & urban & 2,686 & $28.5 \%$ & 631.52 & 4.253 & $63.3 \%$ \\
\hline 37181 & $\mathrm{NC}$ & Vance & rural & 833 & $28.0 \%$ & 253.52 & 3.286 & $56.6 \%$ \\
\hline 37183 & NC & Wake & urban & 13,556 & $28.5 \%$ & 835.22 & 16.230 & $57.8 \%$ \\
\hline 37185 & $\mathrm{NC}$ & Warren & rural & 361 & $27.6 \%$ & 428.46 & 0.843 & $61.4 \%$ \\
\hline 37187 & $\mathrm{NC}$ & Washington & rural & 345 & $28.3 \%$ & 348.13 & 0.991 & $80.8 \%$ \\
\hline 37189 & $\mathrm{NC}$ & Watauga & rural & 898 & $28.7 \%$ & 312.56 & 2.873 & $62.3 \%$ \\
\hline 37191 & $\mathrm{NC}$ & Wayne & urban & 2,238 & $28.5 \%$ & 553.09 & 4.046 & $71.3 \%$ \\
\hline 37193 & NC & Wilkes & rural & 1,202 & $28.4 \%$ & 754.28 & 1.594 & $48.0 \%$ \\
\hline 37195 & $\mathrm{NC}$ & Wilson & rural & 1,659 & $28.3 \%$ & 368.17 & 4.506 & $75.8 \%$ \\
\hline 37197 & $\mathrm{NC}$ & Yadkin & urban & 477 & $28.2 \%$ & 334.83 & 1.423 & $31.6 \%$ \\
\hline 37199 & NC & Yancey & rural & 583 & $28.6 \%$ & 312.60 & 1.866 & $64.9 \%$ \\
\hline 38001 & ND & Adams & rural & 101 & $28.2 \%$ & 987.62 & 0.102 & $85.0 \%$ \\
\hline 38003 & ND & Barnes & rural & 350 & $28.4 \%$ & $1,491.55$ & 0.235 & $73.4 \%$ \\
\hline 38005 & ND & Benson & rural & 136 & $28.4 \%$ & $1,388.71$ & 0.098 & $79.9 \%$ \\
\hline 38009 & ND & Bottineau & rural & 288 & $28.6 \%$ & $1,668.42$ & 0.173 & $90.2 \%$ \\
\hline 38011 & ND & Bowman & rural & 112 & $28.5 \%$ & $1,161.81$ & 0.096 & $83.4 \%$ \\
\hline 38015 & ND & Burleigh & urban & 2,011 & $28.5 \%$ & $1,632.65$ & 1.232 & $68.9 \%$ \\
\hline 38017 & ND & Cass & urban & 2,651 & $28.4 \%$ & $1,764.94$ & 1.502 & $66.5 \%$ \\
\hline 38019 & ND & Cavalier & rural & 169 & $28.3 \%$ & $1,488.75$ & 0.114 & $83.1 \%$ \\
\hline 38021 & ND & Dickey & rural & 173 & $28.4 \%$ & $1,131.47$ & 0.153 & $81.7 \%$ \\
\hline 38029 & ND & Emmons & rural & 113 & $28.5 \%$ & $1,510.43$ & 0.075 & $64.4 \%$ \\
\hline 38031 & ND & Foster & rural & 113 & $28.3 \%$ & 635.45 & 0.178 & $76.9 \%$ \\
\hline 38035 & ND & Grand Forks & urban & 1,269 & $28.4 \%$ & 1436.38 & 0.883 & $72.8 \%$ \\
\hline 38041 & ND & Hettinger & rural & 106 & $28.3 \%$ & $1,132.22$ & 0.093 & $78.9 \%$ \\
\hline 38045 & ND & LaMoure & rural & 145 & $28.5 \%$ & $1,145.94$ & 0.127 & $71.5 \%$ \\
\hline 38049 & ND & McHenry & rural & 183 & $28.5 \%$ & $1,873.95$ & 0.098 & $76.8 \%$ \\
\hline 38051 & ND & McIntosh & rural & 115 & $28.4 \%$ & 974.73 & 0.118 & $66.6 \%$ \\
\hline 38053 & ND & McKenzie & rural & 145 & $28.6 \%$ & $2,760.32$ & 0.053 & $88.2 \%$ \\
\hline 38055 & ND & McLean & rural & 338 & $28.4 \%$ & $2,110.88$ & 0.160 & $77.5 \%$ \\
\hline 38057 & ND & Mercer & rural & 248 & $28.4 \%$ & $1,042.96$ & 0.237 & $77.3 \%$ \\
\hline 38059 & ND & Morton & urban & 675 & $28.6 \%$ & $1,926.27$ & 0.350 & $67.5 \%$ \\
\hline 38061 & ND & Mountrail & rural & 188 & $28.2 \%$ & $1,825.30$ & 0.103 & $86.5 \%$ \\
\hline 38063 & ND & Nelson & rural & 185 & $28.3 \%$ & 981.77 & 0.189 & $92.8 \%$ \\
\hline 38067 & ND & Pembina & rural & 251 & $28.2 \%$ & $1,118.69$ & 0.224 & $78.0 \%$ \\
\hline 38069 & ND & Pierce & rural & 111 & $28.6 \%$ & $1,018.60$ & 0.109 & $62.9 \%$ \\
\hline 38071 & ND & Ramsey & rural & 416 & $28.4 \%$ & $1,186.85$ & 0.350 & $89.8 \%$ \\
\hline
\end{tabular}




\begin{tabular}{|c|c|c|c|c|c|c|c|c|}
\hline 38079 & ND & Rolette & rural & 215 & $28.6 \%$ & 903.08 & 0.238 & $74.7 \%$ \\
\hline 38081 & ND & Sargent & rural & 156 & $28.4 \%$ & 858.51 & 0.181 & $83.5 \%$ \\
\hline 38089 & ND & Stark & rural & 634 & $28.5 \%$ & $1,334.74$ & 0.475 & $78.1 \%$ \\
\hline 38093 & ND & Stutsman & rural & 500 & $28.6 \%$ & $2,221.72$ & 0.225 & $60.9 \%$ \\
\hline 38097 & ND & Traill & rural & 250 & $28.5 \%$ & 861.95 & 0.290 & $78.3 \%$ \\
\hline 38099 & ND & Walsh & rural & 363 & $28.2 \%$ & $1,281.93$ & 0.283 & $79.3 \%$ \\
\hline 38101 & ND & Ward & rural & 1,226 & $28.5 \%$ & $2,013.28$ & 0.609 & $78.0 \%$ \\
\hline 38103 & $\mathrm{ND}$ & Wells & rural & 218 & $28.5 \%$ & $1,271.05$ & 0.172 & $95.4 \%$ \\
\hline 38105 & ND & Williams & rural & 584 & $28.4 \%$ & $2,077.40$ & 0.281 & $89.8 \%$ \\
\hline 31001 & $\mathrm{NE}$ & Adams & rural & 931 & $28.6 \%$ & 563.27 & 1.653 & $87.4 \%$ \\
\hline 31011 & $\mathrm{NE}$ & Boone & rural & 222 & $28.3 \%$ & 686.55 & 0.324 & $91.3 \%$ \\
\hline 31013 & $\mathrm{NE}$ & Box Butte & rural & 339 & $28.5 \%$ & 1075.29 & 0.315 & $89.5 \%$ \\
\hline 31015 & $\mathrm{NE}$ & Boyd & rural & 114 & $28.1 \%$ & 539.94 & 0.211 & $95.3 \%$ \\
\hline 31017 & $\mathrm{NE}$ & Brown & rural & 138 & $28.7 \%$ & $1,221.33$ & 0.113 & $93.2 \%$ \\
\hline 31019 & $\mathrm{NE}$ & Buffalo & rural & 1,166 & $28.6 \%$ & 968.11 & 1.204 & $82.4 \%$ \\
\hline 31021 & $\mathrm{NE}$ & Burt & rural & 271 & $28.5 \%$ & 491.58 & 0.551 & $84.0 \%$ \\
\hline 31023 & $\mathrm{NE}$ & Butler & rural & 310 & $28.6 \%$ & 584.91 & 0.529 & $88.5 \%$ \\
\hline 31025 & $\mathrm{NE}$ & Cass & urban & 682 & $28.5 \%$ & 557.45 & 1.224 & $77.2 \%$ \\
\hline 31027 & $\mathrm{NE}$ & Cedar & rural & 262 & $28.6 \%$ & 740.31 & 0.354 & $72.3 \%$ \\
\hline 31033 & $\mathrm{NE}$ & Cheyenne & rural & 304 & $28.3 \%$ & $1,196.29$ & 0.254 & $86.1 \%$ \\
\hline 31035 & $\mathrm{NE}$ & Clay & rural & 267 & $28.3 \%$ & 572.29 & 0.466 & $91.0 \%$ \\
\hline 31037 & $\mathrm{NE}$ & Colfax & rural & 365 & $28.2 \%$ & 411.66 & 0.885 & $88.9 \%$ \\
\hline 31039 & $\mathrm{NE}$ & Cuming & rural & 343 & $28.7 \%$ & 570.62 & 0.601 & $88.3 \%$ \\
\hline 31041 & $\mathrm{NE}$ & Custer & rural & 436 & $28.5 \%$ & $2,575.52$ & 0.169 & $92.4 \%$ \\
\hline 31043 & $\mathrm{NE}$ & Dakota & urban & 214 & $28.1 \%$ & 264.25 & 0.811 & $59.7 \%$ \\
\hline 31045 & $\mathrm{NE}$ & Dawes & rural & 264 & $28.4 \%$ & $1,396.46$ & 0.189 & $75.4 \%$ \\
\hline 31047 & $\mathrm{NE}$ & Dawson & rural & 603 & $28.4 \%$ & $1,013.10$ & 0.596 & $80.9 \%$ \\
\hline 31051 & $\mathrm{NE}$ & Dixon & urban & 286 & $28.6 \%$ & 476.23 & 0.600 & $64.7 \%$ \\
\hline 31053 & $\mathrm{NE}$ & Dodge & rural & 1,190 & $28.5 \%$ & 528.71 & 2.251 & $81.6 \%$ \\
\hline 31055 & $\mathrm{NE}$ & Douglas & urban & 9,755 & $28.5 \%$ & 328.45 & 29.702 & $65.0 \%$ \\
\hline 31059 & $\mathrm{NE}$ & Fillmore & rural & 256 & $28.3 \%$ & 575.37 & 0.446 & $94.5 \%$ \\
\hline 31061 & $\mathrm{NE}$ & Franklin & rural & 156 & $28.4 \%$ & 575.82 & 0.270 & $93.7 \%$ \\
\hline 31065 & $\mathrm{NE}$ & Furnas & rural & 231 & $28.4 \%$ & 719.13 & 0.322 & $92.3 \%$ \\
\hline 31067 & $\mathrm{NE}$ & Gage & rural & 765 & $28.5 \%$ & 851.49 & 0.898 & $84.7 \%$ \\
\hline 31069 & $\mathrm{NE}$ & Garden & rural & 123 & $28.5 \%$ & $1,704.28$ & 0.072 & $92.7 \%$ \\
\hline 31071 & $\mathrm{NE}$ & Garfield & rural & 109 & $28.3 \%$ & 569.79 & 0.192 & $92.1 \%$ \\
\hline 31077 & $\mathrm{NE}$ & Greeley & rural & 117 & $28.9 \%$ & 569.81 & 0.205 & $94.0 \%$ \\
\hline 31087 & $\mathrm{NE}$ & Hitchcock & rural & 141 & $28.5 \%$ & 709.94 & 0.198 & $93.6 \%$ \\
\hline 31089 & $\mathrm{NE}$ & Holt & rural & 401 & $28.4 \%$ & $2,412.40$ & 0.166 & $93.5 \%$ \\
\hline 31093 & $\mathrm{NE}$ & Howard & urban & 235 & $28.1 \%$ & 569.34 & 0.412 & $90.7 \%$ \\
\hline 31095 & $\mathrm{NE}$ & Jefferson & rural & 306 & $28.6 \%$ & 570.18 & 0.537 & $87.2 \%$ \\
\hline 31097 & $\mathrm{NE}$ & Johnson & rural & 172 & $28.5 \%$ & 376.05 & 0.457 & $89.1 \%$ \\
\hline 31099 & $\mathrm{NE}$ & Kearney & rural & 206 & $28.5 \%$ & 516.24 & 0.400 & $88.8 \%$ \\
\hline 31101 & $\mathrm{NE}$ & Keith & rural & 348 & $28.7 \%$ & $1,061.60$ & 0.328 & $88.0 \%$ \\
\hline 31105 & $\mathrm{NE}$ & Kimball & rural & 157 & $28.6 \%$ & 951.85 & 0.165 & $91.3 \%$ \\
\hline 31107 & $\mathrm{NE}$ & Knox & rural & 361 & $28.6 \%$ & $1,108.35$ & 0.326 & $83.4 \%$ \\
\hline 31109 & $\mathrm{NE}$ & Lancaster & urban & 6,452 & $28.5 \%$ & 837.55 & 7.704 & $78.3 \%$ \\
\hline 31111 & $\mathrm{NE}$ & Lincoln & rural & 956 & $28.6 \%$ & $2,564.07$ & 0.373 & $77.3 \%$ \\
\hline 31119 & $\mathrm{NE}$ & Madison & rural & 1,016 & $28.6 \%$ & 572.74 & 1.774 & $77.7 \%$ \\
\hline 31121 & $\mathrm{NE}$ & Merrick & urban & 244 & $28.9 \%$ & 484.88 & 0.504 & $85.4 \%$ \\
\hline 31123 & $\mathrm{NE}$ & Morrill & rural & 177 & $28.2 \%$ & $1,423.84$ & 0.125 & $86.4 \%$ \\
\hline 31125 & $\mathrm{NE}$ & Nance & rural & 125 & $28.2 \%$ & 441.63 & 0.284 & $88.6 \%$ \\
\hline 31127 & $\mathrm{NE}$ & Nemaha & rural & 227 & $28.6 \%$ & 407.38 & 0.558 & $90.5 \%$ \\
\hline 31129 & $\mathrm{NE}$ & Nuckolls & rural & 198 & $28.4 \%$ & 575.16 & 0.344 & $90.3 \%$ \\
\hline 31131 & $\mathrm{NE}$ & Otoe & rural & 477 & $28.4 \%$ & 615.63 & 0.775 & $83.7 \%$ \\
\hline 31133 & $\mathrm{NE}$ & Pawnee & rural & 126 & $28.5 \%$ & 431.07 & 0.292 & $92.5 \%$ \\
\hline 31135 & $\mathrm{NE}$ & Perkins & rural & 120 & $28.3 \%$ & 883.34 & 0.136 & $91.6 \%$ \\
\hline 31137 & $\mathrm{NE}$ & Phelps & rural & 333 & $28.9 \%$ & 539.79 & 0.616 & $92.8 \%$ \\
\hline 31139 & $\mathrm{NE}$ & Pierce & rural & 229 & $28.4 \%$ & 573.25 & 0.399 & $90.6 \%$ \\
\hline 31141 & $\mathrm{NE}$ & Platte & rural & 906 & $28.6 \%$ & 674.06 & 1.345 & $86.1 \%$ \\
\hline 31143 & $\mathrm{NE}$ & Polk & rural & 179 & $28.5 \%$ & 438.34 & 0.408 & $92.4 \%$ \\
\hline 31145 & $\mathrm{NE}$ & Red Willow & rural & 414 & $28.6 \%$ & 716.99 & 0.578 & $92.5 \%$ \\
\hline 31147 & $\mathrm{NE}$ & Richardson & rural & 344 & $28.8 \%$ & 551.84 & 0.624 & $93.7 \%$ \\
\hline 31151 & $\mathrm{NE}$ & Saline & rural & 441 & $28.7 \%$ & 574.02 & 0.769 & $89.8 \%$ \\
\hline 31153 & $\mathrm{NE}$ & Sarpy & urban & 2,536 & $28.6 \%$ & 238.99 & 10.613 & $75.7 \%$ \\
\hline 31155 & $\mathrm{NE}$ & Saunders & urban & 582 & $28.6 \%$ & 750.23 & 0.776 & $79.2 \%$ \\
\hline 31157 & NE & Scotts Bluff & rural & 1,189 & $28.6 \%$ & 739.4 & 1.608 & $83.2 \%$ \\
\hline 31159 & $\mathrm{NE}$ & Seward & urban & 517 & $28.5 \%$ & 571.43 & 0.904 & $87.6 \%$ \\
\hline 31161 & $\mathrm{NE}$ & Sheridan & rural & 217 & $28.3 \%$ & $2,440.86$ & 0.089 & $80.4 \%$ \\
\hline 31163 & $\mathrm{NE}$ & Sherman & rural & 124 & $28.3 \%$ & 565.83 & 0.219 & $84.1 \%$ \\
\hline 31169 & $\mathrm{NE}$ & Thayer & rural & 255 & $28.8 \%$ & 573.81 & 0.444 & $93.4 \%$ \\
\hline 31173 & $\mathrm{NE}$ & Thurston & rural & 138 & $28.8 \%$ & 393.58 & 0.350 & $79.7 \%$ \\
\hline 31175 & $\mathrm{NE}$ & Valley & rural & 192 & $28.7 \%$ & 568.05 & 0.339 & $93.5 \%$ \\
\hline 31177 & $\mathrm{NE}$ & Washington & urban & 512 & $28.4 \%$ & 389.96 & 1.312 & $75.3 \%$ \\
\hline
\end{tabular}




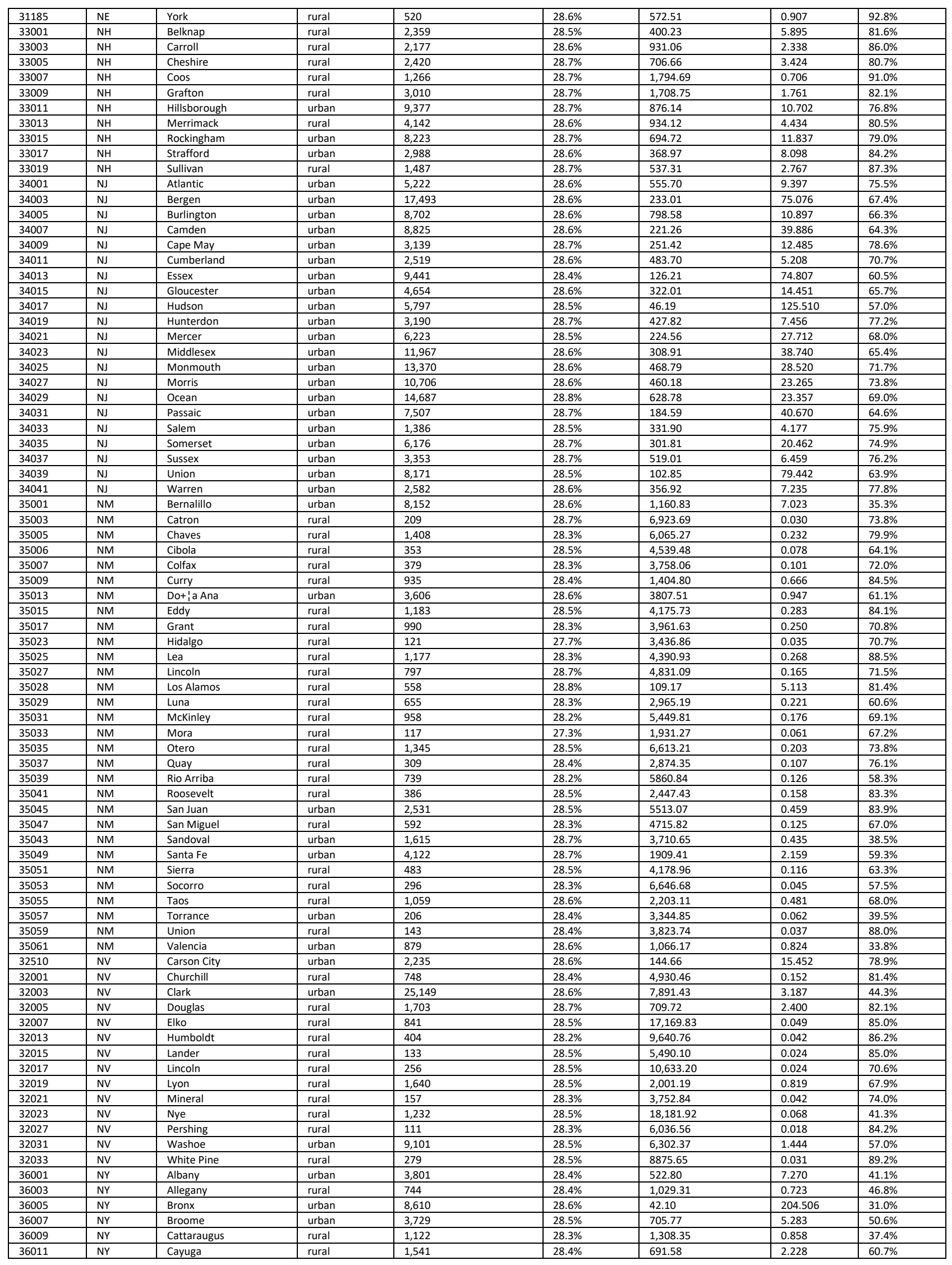




\begin{tabular}{|c|c|c|c|c|c|c|c|c|}
\hline 36013 & NY & Chautauqua & rural & 2,126 & $28.5 \%$ & $1,060.23$ & 2.005 & $39.8 \%$ \\
\hline 36015 & NY & Chemung & urban & 1,617 & $28.4 \%$ & 407.35 & 3.970 & $53.2 \%$ \\
\hline 36017 & NY & Chenango & rural & 983 & $28.5 \%$ & 893.55 & 1.100 & $51.1 \%$ \\
\hline 36019 & NY & Clinton & rural & 1,622 & $28.5 \%$ & $1,037.85$ & 1.562 & $69.2 \%$ \\
\hline 36021 & NY & Columbia & rural & 1,459 & $28.5 \%$ & 634.71 & 2.298 & $57.3 \%$ \\
\hline 36023 & NY & Cortland & rural & 920 & $28.5 \%$ & 498.76 & 1.845 & $61.7 \%$ \\
\hline 36025 & NY & Delaware & rural & 1,106 & $28.5 \%$ & $1,442.44$ & 0.767 & $60.9 \%$ \\
\hline 36027 & NY & Dutchess & urban & 5,767 & $28.7 \%$ & 795.63 & 7.248 & $67.9 \%$ \\
\hline 36029 & NY & Erie & urban & 8,891 & $28.3 \%$ & $1,042.69$ & 8.527 & $25.3 \%$ \\
\hline 36031 & NY & Essex & rural & 1,092 & $28.4 \%$ & $1,794.23$ & 0.609 & $71.0 \%$ \\
\hline 36035 & NY & Fulton & rural & 681 & $28.4 \%$ & 495.47 & 1.375 & $37.4 \%$ \\
\hline 36037 & $\mathrm{NY}$ & Genesee & rural & 633 & $28.2 \%$ & 492.94 & 1.284 & $27.0 \%$ \\
\hline 36039 & NY & Greene & rural & 971 & $28.4 \%$ & 647.16 & 1.501 & $53.0 \%$ \\
\hline 36041 & NY & Hamilton & rural & 187 & $28.7 \%$ & $1,717.37$ & 0.109 & $63.4 \%$ \\
\hline 36043 & NY & Herkimer & urban & 1,113 & $28.5 \%$ & $1,411.47$ & 0.789 & $49.6 \%$ \\
\hline 36045 & NY & Jefferson & urban & 1,819 & $28.5 \%$ & $1,268.59$ & 1.434 & $60.9 \%$ \\
\hline 36047 & NY & Kings & urban & 17,732 & $28.4 \%$ & 70.82 & 250.387 & $36.0 \%$ \\
\hline 36049 & $\mathrm{NY}$ & Lewis & rural & 512 & $28.5 \%$ & $1,274.68$ & 0.401 & $61.6 \%$ \\
\hline 36051 & NY & Livingston & urban & 590 & $28.2 \%$ & 631.76 & 0.933 & $24.5 \%$ \\
\hline 36057 & NY & Montgomery & rural & 888 & $28.2 \%$ & 403.04 & 2.202 & $44.3 \%$ \\
\hline 36059 & NY & Nassau & urban & 20,314 & $28.6 \%$ & 284.72 & 71.349 & $53.3 \%$ \\
\hline 36061 & NY & New York & urban & 22,299 & $28.8 \%$ & 22.83 & 976.758 & $48.7 \%$ \\
\hline 36063 & NY & Niagara & urban & 2,410 & $28.3 \%$ & 522.36 & 4.613 & $29.7 \%$ \\
\hline 36065 & NY & Oneida & urban & 4,209 & $28.5 \%$ & $1,212.43$ & 3.471 & $52.0 \%$ \\
\hline 36067 & NY & Onondaga & urban & 7,872 & $28.5 \%$ & 778.39 & 10.114 & $51.1 \%$ \\
\hline 36069 & NY & Ontario & urban & 1,119 & $28.4 \%$ & 644.07 & 1.738 & $24.3 \%$ \\
\hline 36071 & NY & Orange & urban & 5,548 & $28.6 \%$ & 811.69 & 6.835 & $67.7 \%$ \\
\hline 36073 & NY & Orleans & urban & 407 & $28.3 \%$ & 391.26 & 1.041 & $29.0 \%$ \\
\hline 36075 & NY & Oswego & urban & 1,952 & $28.5 \%$ & 951.65 & 2.051 & $51.8 \%$ \\
\hline 36077 & NY & Otsego & rural & 1,598 & $28.5 \%$ & $1,001.70$ & 1.596 & $65.5 \%$ \\
\hline 36079 & NY & Putnam & urban & 1,801 & $28.7 \%$ & 230.31 & 7.822 & $65.7 \%$ \\
\hline 36081 & NY & Queens & urban & 17,974 & $28.5 \%$ & 108.53 & 165.616 & $33.5 \%$ \\
\hline 36083 & NY & Rensselaer & urban & 2,143 & $28.5 \%$ & 652.43 & 3.285 & $41.1 \%$ \\
\hline 36085 & NY & Richmond & urban & 4,540 & $28.7 \%$ & 58.37 & 77.786 & $36.5 \%$ \\
\hline 36087 & NY & Rockland & urban & 5,214 & $28.7 \%$ & 173.55 & 30.045 & $62.8 \%$ \\
\hline 36091 & NY & Saratoga & urban & 3,493 & $28.5 \%$ & 809.98 & 4.312 & $43.3 \%$ \\
\hline 36093 & NY & Schenectady & urban & 2,494 & $28.4 \%$ & 204.52 & 12.196 & $41.0 \%$ \\
\hline 36089 & NY & St. Lawrence & rural & 2,315 & $28.5 \%$ & 2680.38 & 0.864 & $68.5 \%$ \\
\hline 36101 & NY & Steuben & rural & 1,831 & $28.6 \%$ & $1,390.56$ & 1.317 & $53.8 \%$ \\
\hline 36103 & NY & Suffolk & urban & 23,417 & $28.7 \%$ & 912.05 & 25.675 & $58.8 \%$ \\
\hline 36105 & NY & Sullivan & rural & 1,805 & $28.6 \%$ & 968.13 & 1.865 & $77.1 \%$ \\
\hline 36107 & NY & Tioga & urban & 930 & $28.4 \%$ & 518.60 & 1.794 & $51.7 \%$ \\
\hline 36109 & NY & Tompkins & urban & 1,857 & $28.5 \%$ & 474.65 & 3.912 & $62.9 \%$ \\
\hline 36111 & NY & Ulster & urban & 3,794 & $28.6 \%$ & $1,124.24$ & 3.375 & $63.0 \%$ \\
\hline 36113 & NY & Warren & urban & 1,418 & $28.5 \%$ & 866.95 & 1.635 & $48.1 \%$ \\
\hline 36115 & NY & Washington & urban & 1,101 & $28.4 \%$ & 831.18 & 1.325 & $48.2 \%$ \\
\hline 36117 & NY & Wayne & urban & 846 & $28.3 \%$ & 603.83 & 1.401 & $23.2 \%$ \\
\hline 36119 & NY & Westchester & urban & 16,186 & $28.6 \%$ & 430.50 & 37.599 & $57.2 \%$ \\
\hline 36121 & NY & Wyoming & rural & 403 & $28.4 \%$ & 592.75 & 0.680 & $28.1 \%$ \\
\hline 36123 & NY & Yates & urban & 357 & $28.6 \%$ & 338.14 & 1.056 & $32.6 \%$ \\
\hline 39001 & $\mathrm{OH}$ & Adams & rural & 523 & $28.6 \%$ & 583.87 & 0.896 & $52.2 \%$ \\
\hline 39003 & $\mathrm{OH}$ & Allen & urban & 2,008 & $28.3 \%$ & 402.50 & 4.989 & $62.1 \%$ \\
\hline 39005 & $\mathrm{OH}$ & Ashland & rural & 887 & $28.6 \%$ & 422.95 & 2.096 & $48.4 \%$ \\
\hline 39007 & $\mathrm{OH}$ & Ashtabula & rural & 2,028 & $28.4 \%$ & 701.93 & 2.890 & $59.3 \%$ \\
\hline 39009 & $\mathrm{OH}$ & Athens & rural & 797 & $28.4 \%$ & 503.60 & 1.582 & $46.9 \%$ \\
\hline 39011 & $\mathrm{OH}$ & Auglaize & rural & 1,263 & $28.5 \%$ & 401.39 & 3.145 & $64.0 \%$ \\
\hline 39013 & $\mathrm{OH}$ & Belmont & urban & 986 & $28.3 \%$ & 532.13 & 1.852 & $37.1 \%$ \\
\hline 39015 & $\mathrm{OH}$ & Brown & urban & 591 & $28.6 \%$ & 490.02 & 1.206 & $44.8 \%$ \\
\hline 39017 & $\mathrm{OH}$ & Butler & urban & 4,625 & $28.5 \%$ & 467.06 & 9.902 & $44.7 \%$ \\
\hline 39019 & $\mathrm{OH}$ & Carroll & urban & 332 & $28.6 \%$ & 394.61 & 0.842 & $39.4 \%$ \\
\hline 39021 & $\mathrm{OH}$ & Champaign & rural & 566 & $28.4 \%$ & 428.67 & 1.319 & $42.7 \%$ \\
\hline 39023 & $\mathrm{OH}$ & Clark & urban & 1,984 & $28.4 \%$ & 397.47 & 4.991 & $37.9 \%$ \\
\hline 39025 & $\mathrm{OH}$ & Clermont & urban & 2,042 & $28.5 \%$ & 452.10 & 4.517 & $41.4 \%$ \\
\hline 39027 & $\mathrm{OH}$ & Clinton & rural & 737 & $28.4 \%$ & 408.68 & 1.803 & $49.3 \%$ \\
\hline 39029 & $\mathrm{OH}$ & Columbiana & rural & 1,890 & $28.5 \%$ & 531.89 & 3.554 & $46.7 \%$ \\
\hline 39031 & $\mathrm{OH}$ & Coshocton & rural & 653 & $28.6 \%$ & 563.91 & 1.159 & $59.5 \%$ \\
\hline 39033 & $\mathrm{OH}$ & Crawford & rural & 1,005 & $28.5 \%$ & 401.79 & 2.501 & $60.6 \%$ \\
\hline 39035 & $\mathrm{OH}$ & Cuyahoga & urban & 19,128 & $28.3 \%$ & 457.19 & 41.838 & $44.4 \%$ \\
\hline 39037 & $\mathrm{OH}$ & Darke & rural & 1,155 & $28.6 \%$ & 598.10 & 1.932 & $62.1 \%$ \\
\hline 39039 & $\mathrm{OH}$ & Defiance & rural & 884 & $28.5 \%$ & 411.46 & 2.149 & $65.7 \%$ \\
\hline 39041 & $\mathrm{OH}$ & Delaware & urban & 1,712 & $28.7 \%$ & 443.10 & 3.863 & $42.7 \%$ \\
\hline 39043 & $\mathrm{OH}$ & Erie & rural & 1,999 & $28.6 \%$ & 251.56 & 7.947 & $63.9 \%$ \\
\hline 39045 & $\mathrm{OH}$ & Fairfield & urban & 1,640 & $28.6 \%$ & 504.41 & 3.252 & $36.2 \%$ \\
\hline 39047 & $\mathrm{OH}$ & Fayette & rural & 376 & $28.5 \%$ & 406.36 & 0.926 & $42.0 \%$ \\
\hline
\end{tabular}




\begin{tabular}{|c|c|c|c|c|c|c|c|c|}
\hline 39053 & $\mathrm{OH}$ & Gallia & rural & 632 & $28.6 \%$ & 466.53 & 1.354 & $61.0 \%$ \\
\hline 39055 & $\mathrm{OH}$ & Geauga & urban & 1,741 & $28.7 \%$ & 400.16 & 4.351 & $50.7 \%$ \\
\hline 39057 & $\mathrm{OH}$ & Greene & urban & 1,798 & $28.4 \%$ & 413.73 & 4.346 & $46.6 \%$ \\
\hline 39059 & $\mathrm{OH}$ & Guernsey & rural & 761 & $28.6 \%$ & 522.25 & 1.458 & $54.2 \%$ \\
\hline 39061 & $\mathrm{OH}$ & Hamilton & urban & 12,371 & $28.4 \%$ & 405.91 & 30.477 & $46.2 \%$ \\
\hline 39063 & $\mathrm{OH}$ & Hancock & rural & 1,386 & $28.6 \%$ & 531.36 & 2.608 & $61.0 \%$ \\
\hline 39065 & $\mathrm{OH}$ & Hardin & rural & 595 & $28.5 \%$ & 470.40 & 1.266 & $64.0 \%$ \\
\hline 39067 & $\mathrm{OH}$ & Harrison & rural & 338 & $28.5 \%$ & 402.34 & 0.841 & $52.4 \%$ \\
\hline 39069 & $\mathrm{OH}$ & Henry & rural & 637 & $28.4 \%$ & 416.01 & 1.532 & $64.8 \%$ \\
\hline 39071 & $\mathrm{OH}$ & Highland & rural & 688 & $28.7 \%$ & 553.08 & 1.244 & $54.3 \%$ \\
\hline 39075 & $\mathrm{OH}$ & Holmes & rural & 331 & $28.3 \%$ & 422.53 & 0.785 & $42.8 \%$ \\
\hline 39077 & $\mathrm{OH}$ & Huron & rural & 1,493 & $28.6 \%$ & 491.49 & 3.037 & $67.0 \%$ \\
\hline 39079 & $\mathrm{OH}$ & Jackson & rural & 558 & $28.5 \%$ & 420.30 & 1.327 & $59.0 \%$ \\
\hline 39081 & $\mathrm{OH}$ & Jefferson & urban & 1,357 & $28.4 \%$ & 408.33 & 3.324 & $49.8 \%$ \\
\hline 39083 & $\mathrm{OH}$ & Knox & rural & 1,075 & $28.5 \%$ & 525.49 & 2.045 & $51.9 \%$ \\
\hline 39085 & $\mathrm{OH}$ & Lake & urban & 4,229 & $28.6 \%$ & 227.49 & 18.588 & $46.6 \%$ \\
\hline 39087 & $\mathrm{OH}$ & Lawrence & urban & 1,271 & $28.5 \%$ & 453.37 & 2.803 & $65.0 \%$ \\
\hline 39089 & $\mathrm{OH}$ & Licking & urban & 2,205 & $28.5 \%$ & 682.50 & 3.231 & $41.7 \%$ \\
\hline 39091 & $\mathrm{OH}$ & Logan & rural & 1,005 & $28.4 \%$ & 458.43 & 2.193 & $59.8 \%$ \\
\hline 39097 & $\mathrm{OH}$ & Madison & urban & 549 & $28.4 \%$ & 465.88 & 1.179 & $36.5 \%$ \\
\hline 39099 & $\mathrm{OH}$ & Mahoning & urban & 3,329 & $28.3 \%$ & 411.62 & 8.088 & $36.7 \%$ \\
\hline 39101 & $\mathrm{OH}$ & Marion & rural & 1,084 & $28.4 \%$ & 403.76 & 2.684 & $50.9 \%$ \\
\hline 39103 & $\mathrm{OH}$ & Medina & urban & 2,836 & $28.6 \%$ & 421.36 & 6.731 & $45.6 \%$ \\
\hline 39105 & $\mathrm{OH}$ & Meigs & rural & 431 & $28.4 \%$ & 430.10 & 1.003 & $62.2 \%$ \\
\hline 39107 & $\mathrm{OH}$ & Mercer & rural & 868 & $28.4 \%$ & 462.45 & 1.876 & $62.5 \%$ \\
\hline 39109 & $\mathrm{OH}$ & Miami & urban & 1,910 & $28.5 \%$ & 406.58 & 4.697 & $48.2 \%$ \\
\hline 39111 & $\mathrm{OH}$ & Monroe & rural & 260 & $28.3 \%$ & 455.72 & 0.570 & $41.1 \%$ \\
\hline 39113 & $\mathrm{OH}$ & Montgomery & urban & 8,092 & $28.4 \%$ & 461.55 & 17.531 & $40.2 \%$ \\
\hline 39115 & $\mathrm{OH}$ & Morgan & rural & 246 & $28.3 \%$ & 416.42 & 0.591 & $52.4 \%$ \\
\hline 39117 & $\mathrm{OH}$ & Morrow & urban & 415 & $28.6 \%$ & 406.08 & 1.021 & $50.5 \%$ \\
\hline 39119 & $\mathrm{OH}$ & Muskingum & rural & 1,654 & $28.5 \%$ & 664.58 & 2.489 & $54.7 \%$ \\
\hline 39121 & $\mathrm{OH}$ & Noble & rural & 163 & $28.5 \%$ & 398.01 & 0.410 & $52.9 \%$ \\
\hline 39123 & $\mathrm{OH}$ & Ottawa & rural & 1,109 & $28.5 \%$ & 254.92 & 4.352 & $57.6 \%$ \\
\hline 39125 & $\mathrm{OH}$ & $\begin{array}{l}\text { Paulding } \\
\end{array}$ & rural & 350 & $28.3 \%$ & 416.44 & 0.841 & $60.0 \%$ \\
\hline 39127 & $\mathrm{OH}$ & Perry & urban & 651 & $28.4 \%$ & 407.97 & 1.595 & $53.6 \%$ \\
\hline 39129 & $\mathrm{OH}$ & Pickaway & urban & 660 & $28.2 \%$ & 501.32 & 1.317 & $39.0 \%$ \\
\hline 39131 & $\mathrm{OH}$ & Pike & rural & 435 & $28.5 \%$ & 440.28 & 0.989 & $58.1 \%$ \\
\hline 39139 & $\mathrm{OH}$ & Richland & urban & 2,824 & $28.6 \%$ & 495.27 & 5.702 & $62.1 \%$ \\
\hline 39141 & $\mathrm{OH}$ & Ross & rural & 1,249 & $28.5 \%$ & 689.19 & 1.813 & $47.8 \%$ \\
\hline 39143 & $\mathrm{OH}$ & Sandusky & rural & 1,046 & $28.5 \%$ & 408.45 & 2.562 & $59.9 \%$ \\
\hline 39145 & $\mathrm{OH}$ & Scioto & rural & 1,422 & $28.5 \%$ & 610.21 & 2.330 & $62.1 \%$ \\
\hline 39147 & $\mathrm{OH}$ & Seneca & rural & 1,371 & $28.6 \%$ & 551.02 & 2.488 & $67.8 \%$ \\
\hline 39149 & $\mathrm{OH}$ & Shelby & rural & 847 & $28.4 \%$ & 407.68 & 2.077 & $58.5 \%$ \\
\hline 39151 & $\mathrm{OH}$ & Stark & urban & 5,565 & $28.4 \%$ & 575.27 & 9.675 & $35.0 \%$ \\
\hline 39153 & $\mathrm{OH}$ & Summit & urban & 6,703 & $28.3 \%$ & 412.75 & 16.241 & $35.6 \%$ \\
\hline 39155 & $\mathrm{OH}$ & Trumbull & urban & 3,347 & $28.5 \%$ & 618.30 & 5.413 & $40.8 \%$ \\
\hline 39157 & $\mathrm{OH}$ & Tuscarawas & rural & 1,535 & $28.5 \%$ & 567.64 & 2.705 & $41.9 \%$ \\
\hline 39159 & $\mathrm{OH}$ & Union & urban & 482 & $28.5 \%$ & 431.73 & 1.118 & $45.2 \%$ \\
\hline 39161 & $\mathrm{OH}$ & Van Wert & rural & 524 & $28.5 \%$ & 409.16 & 1.281 & $61.9 \%$ \\
\hline 39163 & $\mathrm{OH}$ & Vinton & rural & 190 & $28.1 \%$ & 412.36 & 0.461 & $55.3 \%$ \\
\hline 39165 & $\mathrm{OH}$ & Warren & urban & 2,632 & $28.6 \%$ & 401.31 & 6.559 & $44.6 \%$ \\
\hline 39167 & $\mathrm{OH}$ & Washington & rural & 1,587 & $28.7 \%$ & 631.97 & 2.510 & $67.4 \%$ \\
\hline 39169 & $\mathrm{OH}$ & Wayne & rural & 1,885 & $28.4 \%$ & 554.93 & 3.397 & $44.5 \%$ \\
\hline 39171 & $\mathrm{OH}$ & Williams & rural & 775 & $28.7 \%$ & 420.97 & 1.841 & $56.0 \%$ \\
\hline 39173 & $\mathrm{OH}$ & Wood & urban & 1,739 & $28.4 \%$ & 617.20 & 2.817 & $45.2 \%$ \\
\hline 39175 & $\mathrm{OH}$ & Wyandot & rural & 501 & $28.4 \%$ & 406.86 & 1.230 & $61.5 \%$ \\
\hline 40001 & $\mathrm{OK}$ & Adair & rural & 442 & $28.3 \%$ & 573.48 & 0.771 & $81.6 \%$ \\
\hline 40003 & $\mathrm{OK}$ & Alfalfa & rural & 189 & $28.7 \%$ & 866.45 & 0.218 & $91.1 \%$ \\
\hline 40005 & $\mathrm{OK}$ & Atoka & rural & 342 & $28.5 \%$ & 975.52 & 0.350 & $86.8 \%$ \\
\hline 40007 & $\mathrm{OK}$ & Beaver & rural & 149 & $28.3 \%$ & $1,814.67$ & 0.082 & $86.7 \%$ \\
\hline 40009 & $\mathrm{OK}$ & Beckham & rural & 446 & $28.5 \%$ & 901.81 & 0.495 & $86.8 \%$ \\
\hline 40011 & OK & Blaine & rural & 315 & $28.6 \%$ & 928.42 & 0.340 & $87.7 \%$ \\
\hline 40013 & $\mathrm{OK}$ & Bryan & rural & 1,163 & $28.6 \%$ & 904.47 & 1.286 & $84.9 \%$ \\
\hline 40015 & $\mathrm{OK}$ & Caddo & rural & 811 & $28.8 \%$ & $1,278.29$ & 0.634 & $85.9 \%$ \\
\hline 40017 & $\mathrm{OK}$ & Canadian & urban & 1,947 & $28.7 \%$ & 896.63 & 2.171 & $61.8 \%$ \\
\hline 40019 & $\mathrm{OK}$ & Carter & rural & 1,399 & $28.6 \%$ & 822.18 & 1.701 & $85.1 \%$ \\
\hline 40021 & OK & Cherokee & rural & 979 & $28.8 \%$ & 749.41 & 1.307 & $78.5 \%$ \\
\hline 40023 & $\mathrm{OK}$ & Choctaw & rural & 453 & $28.6 \%$ & 770.36 & 0.588 & $85.5 \%$ \\
\hline 40027 & $\mathrm{OK}$ & Cleveland & urban & 3,789 & $28.8 \%$ & 538.77 & 7.033 & $70.7 \%$ \\
\hline 40029 & $\mathrm{OK}$ & Coal & rural & 160 & $28.2 \%$ & 516.68 & 0.309 & $86.2 \%$ \\
\hline 40031 & $\mathrm{OK}$ & Comanche & urban & 2,218 & $28.5 \%$ & $1,069.29$ & 2.074 & $87.5 \%$ \\
\hline 40033 & OK & Cotton & urban & 169 & $29.0 \%$ & 632.65 & 0.266 & $86.6 \%$ \\
\hline 40035 & OK & Craig & rural & 615 & $28.6 \%$ & 761.35 & 0.807 & $81.6 \%$ \\
\hline 40037 & OK & Creek & urban & 1,310 & $28.5 \%$ & 950.14 & 1.379 & $54.6 \%$ \\
\hline
\end{tabular}




\begin{tabular}{|c|c|c|c|c|c|c|c|c|}
\hline 40043 & OK & Dewey & rural & 168 & $28.9 \%$ & 999.48 & 0.169 & $86.4 \%$ \\
\hline 40045 & OK & Ellis & rural & 135 & $28.6 \%$ & $1,231.52$ & 0.110 & $84.2 \%$ \\
\hline 40047 & OK & Garfield & rural & 1,681 & $28.6 \%$ & $1,058.47$ & 1.588 & $85.8 \%$ \\
\hline 40049 & OK & Garvin & rural & 898 & $28.7 \%$ & 802.12 & 1.119 & $84.1 \%$ \\
\hline 40051 & OK & Grady & urban & 1,083 & $28.7 \%$ & $1,100.50$ & 0.984 & $79.0 \%$ \\
\hline 40053 & OK & Grant & rural & 154 & $28.7 \%$ & $1,000.87$ & 0.153 & $89.6 \%$ \\
\hline 40055 & OK & Greer & rural & 183 & $28.7 \%$ & 639.32 & 0.287 & $93.8 \%$ \\
\hline 40059 & OK & Harper & rural & 129 & $28.1 \%$ & $1,039.02$ & 0.125 & $89.5 \%$ \\
\hline 40061 & OK & Haskell & rural & 389 & $28.9 \%$ & 576.52 & 0.674 & $82.7 \%$ \\
\hline 40063 & OK & Hughes & rural & 339 & $28.3 \%$ & 804.65 & 0.421 & $79.9 \%$ \\
\hline 40067 & OK & Jefferson & rural & 203 & $28.7 \%$ & 758.83 & 0.268 & $85.8 \%$ \\
\hline 40069 & OK & Johnston & rural & 295 & $28.9 \%$ & 642.94 & 0.459 & $85.7 \%$ \\
\hline 40071 & OK & Kay & rural & 1,429 & $28.5 \%$ & 919.73 & 1.553 & $83.8 \%$ \\
\hline 40073 & OK & Kingfisher & rural & 403 & $28.6 \%$ & 898.16 & 0.449 & $84.0 \%$ \\
\hline 40075 & OK & Kiowa & rural & 267 & $28.5 \%$ & $1,015.23$ & 0.263 & $85.4 \%$ \\
\hline 40077 & OK & Latimer & rural & 225 & $28.7 \%$ & 722.08 & 0.312 & $84.2 \%$ \\
\hline 40079 & OK & Le Flore & urban & 1,200 & $28.5 \%$ & 1589.21 & 0.755 & $77.4 \%$ \\
\hline 40081 & OK & Lincoln & urban & 794 & $28.7 \%$ & 952.31 & 0.834 & $73.2 \%$ \\
\hline 40083 & $\mathrm{OK}$ & Logan & urban & 661 & $28.5 \%$ & 743.83 & 0.889 & $70.0 \%$ \\
\hline 40095 & OK & Marshall & rural & 499 & $28.8 \%$ & 371.08 & 1.345 & $84.8 \%$ \\
\hline 40097 & OK & Mayes & rural & 1,056 & $28.6 \%$ & 655.39 & 1.612 & $75.8 \%$ \\
\hline 40087 & OK & McClain & urban & 972 & $28.8 \%$ & 570.70 & 1.702 & $77.5 \%$ \\
\hline 40089 & OK & McCurtain & rural & 856 & $28.6 \%$ & $1,850.01$ & 0.463 & $83.9 \%$ \\
\hline 40091 & OK & McIntosh & rural & 743 & $28.8 \%$ & 618.50 & 1.201 & $82.2 \%$ \\
\hline 40099 & OK & Murray & rural & 400 & $28.4 \%$ & 416.46 & 0.962 & $87.6 \%$ \\
\hline 40101 & OK & Muskogee & rural & 1,771 & $28.4 \%$ & 810.45 & 2.185 & $77.0 \%$ \\
\hline 40103 & OK & Noble & rural & 302 & $28.5 \%$ & 731.90 & 0.413 & $88.6 \%$ \\
\hline 40105 & OK & Nowata & rural & 317 & $28.4 \%$ & 565.78 & 0.560 & $85.6 \%$ \\
\hline 40107 & OK & Okfuskee & rural & 289 & $28.5 \%$ & 618.57 & 0.467 & $79.4 \%$ \\
\hline 40109 & OK & Oklahoma & urban & 14,348 & $28.6 \%$ & 708.82 & 20.242 & $63.6 \%$ \\
\hline 40111 & OK & Okmulgee & urban & 937 & $28.4 \%$ & 697.35 & 1.344 & $75.6 \%$ \\
\hline 40113 & OK & Osage & urban & 483 & $28.5 \%$ & $2,246.36$ & 0.215 & $75.0 \%$ \\
\hline 40115 & OK & Ottawa & rural & 907 & $28.6 \%$ & 470.82 & 1.927 & $81.7 \%$ \\
\hline 40117 & OK & Pawnee & urban & 455 & $28.7 \%$ & 567.95 & 0.801 & $78.5 \%$ \\
\hline 40119 & OK & Payne & rural & 1,721 & $28.8 \%$ & 684.70 & 2.514 & $86.1 \%$ \\
\hline 40121 & OK & Pittsburg & rural & 1,271 & $28.5 \%$ & $1,305.46$ & 0.973 & $82.6 \%$ \\
\hline 40123 & OK & Pontotoc & rural & 978 & $28.7 \%$ & 720.44 & 1.357 & $82.8 \%$ \\
\hline 40131 & OK & Rogers & urban & 1,500 & $28.7 \%$ & 675.63 & 2.220 & $57.4 \%$ \\
\hline 40133 & OK & Seminole & rural & 609 & $28.5 \%$ & 632.84 & 0.962 & $78.2 \%$ \\
\hline 40135 & OK & Sequoyah & urban & 1,025 & $28.7 \%$ & 673.27 & 1.523 & $74.1 \%$ \\
\hline 40137 & OK & Stephens & rural & 1,189 & $28.7 \%$ & 870.24 & 1.366 & $81.2 \%$ \\
\hline 40139 & OK & Texas & rural & 359 & $28.4 \%$ & $2,041.26$ & 0.176 & $82.3 \%$ \\
\hline 40141 & OK & Tillman & rural & 195 & $28.6 \%$ & 871.13 & 0.224 & $86.8 \%$ \\
\hline 40143 & OK & Tulsa & urban & 11,866 & $28.6 \%$ & 570.25 & 20.808 & $54.4 \%$ \\
\hline 40145 & OK & Wagoner & urban & 651 & $28.6 \%$ & 561.56 & 1.159 & $56.2 \%$ \\
\hline 40147 & OK & Washington & rural & 1,707 & $28.6 \%$ & 415.45 & 4.108 & $85.1 \%$ \\
\hline 40149 & OK & Washita & rural & 352 & $28.6 \%$ & $1,003.17$ & 0.351 & $87.4 \%$ \\
\hline 40151 & OK & Woods & rural & 263 & $28.5 \%$ & $1,286.45$ & 0.205 & $89.5 \%$ \\
\hline 40153 & OK & Woodward & rural & 500 & $28.6 \%$ & $1,242.40$ & 0.403 & $88.0 \%$ \\
\hline 41001 & $\mathrm{OR}$ & Baker & rural & 776 & $28.6 \%$ & $3,068.36$ & 0.253 & $87.3 \%$ \\
\hline 41003 & $\mathrm{OR}$ & Benton & urban & 1,157 & $28.5 \%$ & 675.94 & 1.711 & $42.4 \%$ \\
\hline 41005 & $\mathrm{OR}$ & Clackamas & urban & 4,492 & $28.5 \%$ & $1,870.32$ & 2.402 & $27.3 \%$ \\
\hline 41007 & $\mathrm{OR}$ & Clatsop & rural & 1,105 & $28.7 \%$ & 829.05 & 1.333 & $63.9 \%$ \\
\hline 41009 & $\mathrm{OR}$ & Columbia & urban & 721 & $28.5 \%$ & 657.36 & 1.097 & $34.9 \%$ \\
\hline 41011 & OR & Coos & rural & 2,365 & $28.6 \%$ & $1,596.17$ & 1.482 & $78.8 \%$ \\
\hline 41013 & OR & Crook & rural & 818 & $28.7 \%$ & $2,979.09$ & 0.275 & $69.6 \%$ \\
\hline 41015 & OR & Curry & rural & 1,154 & $28.6 \%$ & $1,627.46$ & 0.709 & $79.9 \%$ \\
\hline 41017 & $\mathrm{OR}$ & Deschutes & urban & 5,013 & $28.7 \%$ & $3,018.19$ & 1.661 & $62.8 \%$ \\
\hline 41019 & $\mathrm{OR}$ & Douglas & rural & 3,228 & $28.6 \%$ & $5,036.07$ & 0.641 & $57.4 \%$ \\
\hline 41023 & $\mathrm{OR}$ & Grant & rural & 291 & $28.3 \%$ & $4,528.54$ & 0.064 & $71.4 \%$ \\
\hline 41025 & $\mathrm{OR}$ & Harney & rural & 285 & $28.4 \%$ & $10,133.17$ & 0.028 & $84.0 \%$ \\
\hline 41027 & OR & Hood River & rural & 486 & $28.4 \%$ & 521.95 & 0.930 & $63.7 \%$ \\
\hline 41029 & OR & Jackson & urban & 5,945 & $28.6 \%$ & $2,783.55$ & 2.136 & $59.0 \%$ \\
\hline 41031 & $\mathrm{OR}$ & Jefferson & rural & 612 & $28.5 \%$ & $1,780.78$ & 0.344 & $63.4 \%$ \\
\hline 41033 & $\mathrm{OR}$ & Josephine & urban & 2,548 & $28.6 \%$ & $1,639.67$ & 1.554 & $53.3 \%$ \\
\hline 41035 & $\mathrm{OR}$ & Klamath & rural & 1,888 & $28.5 \%$ & $5,941.05$ & 0.318 & $69.3 \%$ \\
\hline 41037 & OR & Lake & rural & 331 & $28.4 \%$ & $8,138.98$ & 0.041 & $84.7 \%$ \\
\hline 41039 & OR & Lane & urban & 6,028 & $28.5 \%$ & $4,553.12$ & 1.324 & $39.0 \%$ \\
\hline 41041 & OR & Lincoln & rural & 1,783 & $28.8 \%$ & 979.77 & 1.819 & $68.8 \%$ \\
\hline 41043 & $\mathrm{OR}$ & Linn & urban & 2,014 & $28.5 \%$ & $2,290.13$ & 0.879 & $37.6 \%$ \\
\hline 41045 & OR & Malheur & rural & 762 & $28.6 \%$ & $9,887.53$ & 0.077 & $81.1 \%$ \\
\hline 41047 & OR & Marion & urban & 3,411 & $28.5 \%$ & $1,182.33$ & 2.885 & $29.3 \%$ \\
\hline 41049 & OR & Morrow & rural & 256 & $28.8 \%$ & $2,031.61$ & 0.126 & $76.0 \%$ \\
\hline 41051 & OR & Multnomah & urban & 7,036 & $28.4 \%$ & 431.30 & 16.314 & $28.8 \%$ \\
\hline
\end{tabular}




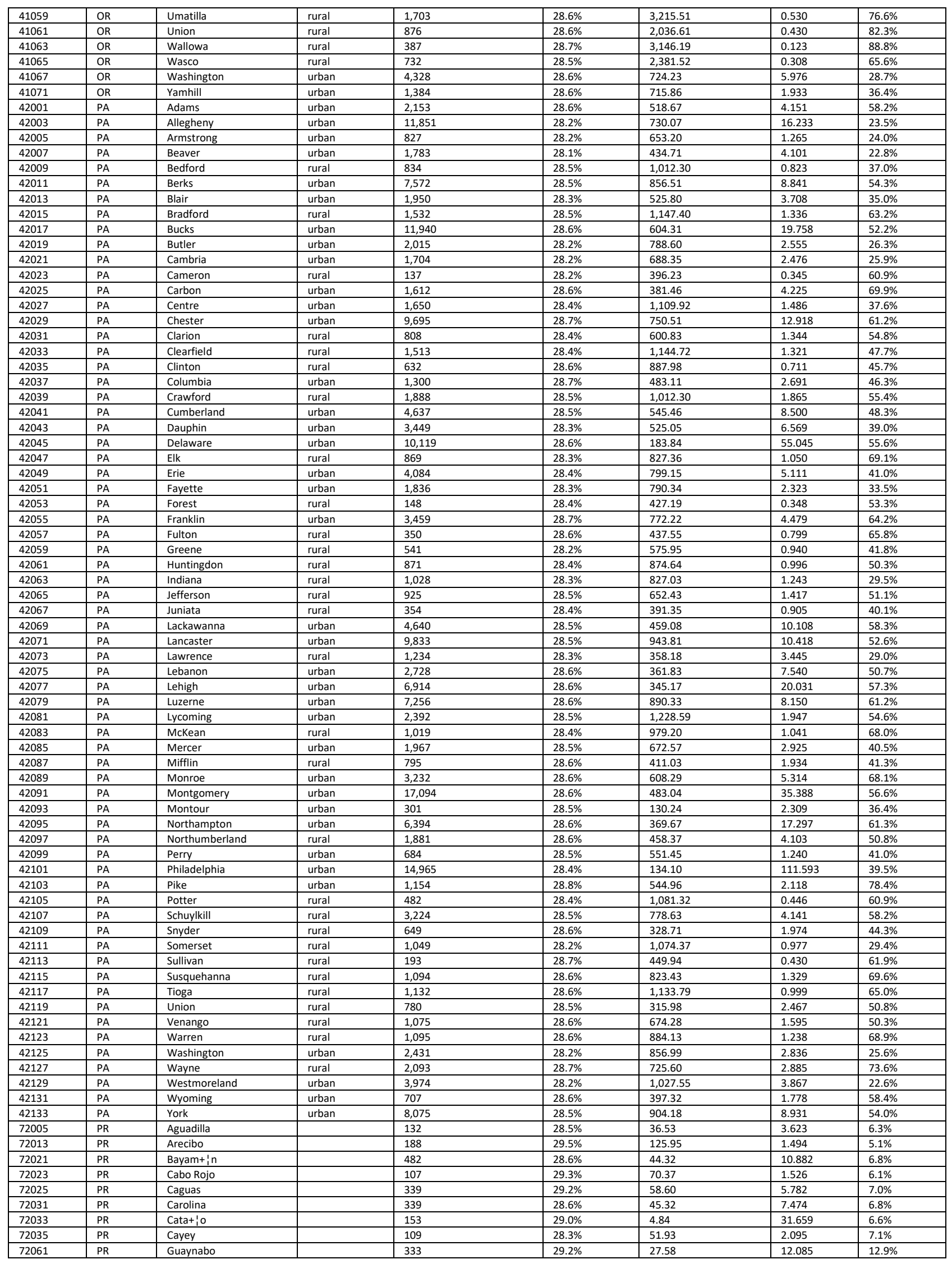




\begin{tabular}{|c|c|c|c|c|c|c|c|c|}
\hline 72069 & $P R$ & Humacao & & 132 & $28.3 \%$ & 44.75 & 2.949 & $7.3 \%$ \\
\hline 72087 & $P R$ & Lo+iza & & 104 & $29.5 \%$ & 19.37 & 5.385 & $5.6 \%$ \\
\hline 72097 & PR & Mayag++ez & & 193 & $29.0 \%$ & 77.65 & 2.484 & $5.9 \%$ \\
\hline 72113 & PR & Ponce & & 411 & $28.9 \%$ & 114.76 & 3.580 & $7.1 \%$ \\
\hline 72127 & PR & San Juan & & 1,645 & $28.8 \%$ & 47.85 & 34.380 & $11.7 \%$ \\
\hline 72139 & PR & Trujillo Alto & & 128 & $29.9 \%$ & 20.76 & 6.176 & $7.7 \%$ \\
\hline 44001 & $\mathrm{RI}$ & Bristol & urban & 874 & $28.7 \%$ & 24.16 & 36.160 & $42.3 \%$ \\
\hline 44003 & $\mathrm{RI}$ & Kent & urban & 2,640 & $28.7 \%$ & 168.53 & 15.665 & $40.5 \%$ \\
\hline 44005 & $\mathrm{RI}$ & Newport & urban & 2,055 & $28.6 \%$ & 102.39 & 20.067 & $58.4 \%$ \\
\hline 44007 & $\mathrm{RI}$ & Providence & urban & 7,687 & $28.6 \%$ & 409.50 & 18.771 & $40.2 \%$ \\
\hline 45001 & SC & Abbeville & rural & 491 & $28.1 \%$ & 490.48 & 1.001 & $68.0 \%$ \\
\hline 45003 & $\mathrm{SC}$ & Aiken & urban & 4,195 & $28.5 \%$ & $1,071.03$ & 3.916 & $71.7 \%$ \\
\hline 45005 & SC & Allendale & rural & 152 & $28.1 \%$ & 408.09 & 0.371 & $60.6 \%$ \\
\hline 45007 & SC & Anderson & urban & 3,894 & $28.5 \%$ & 715.43 & 5.444 & $63.7 \%$ \\
\hline 45009 & SC & Bamberg & rural & 273 & $28.4 \%$ & 393.37 & 0.693 & $66.8 \%$ \\
\hline 45011 & SC & Barnwell & rural & 441 & $28.5 \%$ & 548.39 & 0.805 & $69.5 \%$ \\
\hline 45013 & SC & Beaufort & urban & 7,151 & $28.8 \%$ & 576.28 & 12.409 & $78.7 \%$ \\
\hline 45015 & SC & Berkeley & urban & 2,574 & $28.5 \%$ & $1,098.86$ & 2.343 & $71.2 \%$ \\
\hline 45017 & SC & Calhoun & urban & 236 & $28.6 \%$ & 381.15 & 0.619 & $68.4 \%$ \\
\hline 45023 & SC & Chester & urban & 730 & $28.4 \%$ & 580.66 & 1.257 & $72.4 \%$ \\
\hline 45025 & SC & Chesterfield & rural & 927 & $28.4 \%$ & 799.08 & 1.160 & $79.2 \%$ \\
\hline 45027 & SC & Clarendon & rural & 804 & $28.5 \%$ & 606.94 & 1.325 & $71.3 \%$ \\
\hline 45029 & $\mathrm{SC}$ & Colleton & rural & 762 & $28.4 \%$ & $1,056.49$ & 0.721 & $67.8 \%$ \\
\hline 45031 & SC & Darlington & urban & 1,470 & $28.4 \%$ & 561.15 & 2.619 & $79.6 \%$ \\
\hline 45033 & SC & Dillon & rural & 592 & $28.4 \%$ & 404.87 & 1.462 & $77.8 \%$ \\
\hline 45035 & SC & Dorchester & urban & 2,457 & $28.6 \%$ & 573.23 & 4.285 & $71.1 \%$ \\
\hline 45037 & SC & Edgefield & urban & 364 & $28.1 \%$ & 500.41 & 0.728 & $67.8 \%$ \\
\hline 45039 & SC & Fairfield & urban & 459 & $28.2 \%$ & 686.28 & 0.669 & $65.9 \%$ \\
\hline 45041 & SC & Florence & urban & 3,352 & $28.4 \%$ & 799.96 & 4.191 & $81.8 \%$ \\
\hline 45043 & SC & Georgetown & rural & 2,454 & $28.6 \%$ & 813.55 & 3.016 & $79.4 \%$ \\
\hline 45045 & SC & Greenville & urban & 9,209 & $28.6 \%$ & 785.12 & 11.730 & $62.3 \%$ \\
\hline 45047 & SC & Greenwood & rural & 1,842 & $28.5 \%$ & 454.73 & 4.050 & $72.8 \%$ \\
\hline 45049 & SC & Hampton & rural & 463 & $27.9 \%$ & 559.90 & 0.827 & $73.4 \%$ \\
\hline 45051 & SC & Horry & urban & 10,098 & $28.7 \%$ & $1,133.90$ & 8.905 & $78.2 \%$ \\
\hline 45053 & SC & Jasper & urban & 376 & $28.2 \%$ & 655.32 & 0.574 & $64.9 \%$ \\
\hline 45055 & SC & Kershaw & urban & 1,581 & $28.5 \%$ & 726.56 & 2.176 & $75.4 \%$ \\
\hline 45057 & SC & Lancaster & urban & 1,461 & $28.4 \%$ & 549.16 & 2.661 & $79.6 \%$ \\
\hline 45067 & SC & Marion & rural & 794 & $28.4 \%$ & 489.23 & 1.623 & $79.8 \%$ \\
\hline 45069 & SC & Marlboro & rural & 548 & $28.1 \%$ & 479.67 & 1.142 & $74.4 \%$ \\
\hline 45065 & SC & McCormick & rural & 403 & $28.6 \%$ & 359.13 & 1.121 & $65.9 \%$ \\
\hline 45071 & SC & Newberry & rural & 974 & $28.5 \%$ & 630.04 & 1.545 & $72.3 \%$ \\
\hline 45073 & SC & Oconee & rural & 2,270 & $28.6 \%$ & 626.33 & 3.624 & $71.9 \%$ \\
\hline 45075 & SC & Orangeburg & rural & 1,720 & $28.3 \%$ & $1,106.10$ & 1.555 & $63.3 \%$ \\
\hline 45077 & SC & Pickens & urban & 2,626 & $28.6 \%$ & 496.41 & 5.289 & $61.8 \%$ \\
\hline 45079 & SC & Richland & urban & 6,590 & $28.5 \%$ & 757.07 & 8.705 & $72.2 \%$ \\
\hline 45081 & SC & Saluda & urban & 315 & $28.4 \%$ & 452.78 & 0.696 & $68.2 \%$ \\
\hline 45083 & SC & Spartanburg & urban & 5,994 & $28.5 \%$ & 807.93 & 7.419 & $59.1 \%$ \\
\hline 45085 & SC & Sumter & urban & 2,191 & $28.2 \%$ & 665.07 & 3.294 & $78.2 \%$ \\
\hline 45087 & $\mathrm{SC}$ & Union & urban & 599 & $28.2 \%$ & 514.17 & 1.165 & $65.5 \%$ \\
\hline 45089 & SC & Williamsburg & rural & 639 & $28.1 \%$ & 934.16 & 0.684 & $71.9 \%$ \\
\hline 45091 & SC & York & urban & 5,962 & $28.7 \%$ & 680.59 & 8.760 & $70.6 \%$ \\
\hline 46005 & SD & Beadle & rural & 487 & $28.5 \%$ & $1,258.71$ & 0.387 & $72.4 \%$ \\
\hline 46009 & SD & Bon Homme & rural & 239 & $28.6 \%$ & 563.7 & 0.423 & $78.2 \%$ \\
\hline 46011 & SD & Brookings & rural & 552 & $28.5 \%$ & 792.21 & 0.697 & $72.0 \%$ \\
\hline 46013 & SD & Brown & rural & 1,028 & $28.4 \%$ & $1,712.98$ & 0.600 & $82.3 \%$ \\
\hline 46015 & SD & Brule & rural & 156 & $28.4 \%$ & 817.24 & 0.191 & $77.2 \%$ \\
\hline 46019 & SD & Butte & rural & 274 & $28.3 \%$ & $2,249.90$ & 0.122 & $65.6 \%$ \\
\hline 46023 & SD & Charles Mix & rural & 262 & $28.7 \%$ & 1097.49 & 0.239 & $83.1 \%$ \\
\hline 46027 & SD & Clay & rural & 254 & $28.3 \%$ & 412.19 & 0.617 & $75.0 \%$ \\
\hline 46029 & SD & Codington & rural & 509 & $28.4 \%$ & 688.50 & 0.740 & $53.8 \%$ \\
\hline 46033 & SD & Custer & urban & 356 & $28.8 \%$ & $1,557.00$ & 0.229 & $74.0 \%$ \\
\hline 46035 & SD & Davison & rural & 571 & $28.6 \%$ & 435.56 & 1.312 & $79.7 \%$ \\
\hline 46037 & SD & Day & rural & 197 & $28.5 \%$ & $1,027.87$ & 0.192 & $70.4 \%$ \\
\hline 46039 & SD & Deuel & rural & 113 & $28.5 \%$ & 622.69 & 0.181 & $62.2 \%$ \\
\hline 46043 & SD & Douglas & rural & 124 & $28.4 \%$ & 431.80 & 0.286 & $78.5 \%$ \\
\hline 46045 & SD & Edmunds & rural & 148 & $28.8 \%$ & $1,125.96$ & 0.131 & $85.1 \%$ \\
\hline 46047 & SD & Fall River & rural & 340 & $28.4 \%$ & 1739.92 & 0.195 & $72.9 \%$ \\
\hline 46051 & SD & Grant & rural & 188 & $28.4 \%$ & 681.46 & 0.276 & $55.9 \%$ \\
\hline 46053 & SD & Gregory & rural & 183 & $28.4 \%$ & $1,014.96$ & 0.181 & $78.7 \%$ \\
\hline 46057 & SD & Hamlin & rural & 126 & $28.4 \%$ & 507.23 & 0.249 & $61.8 \%$ \\
\hline 46059 & SD & Hand & rural & 108 & $28.2 \%$ & $1,436.61$ & 0.075 & $83.7 \%$ \\
\hline 46061 & $S D$ & Hanson & rural & 210 & $28.7 \%$ & 434.51 & 0.484 & $80.5 \%$ \\
\hline 46065 & $S D$ & Hughes & rural & 470 & $28.3 \%$ & 741.56 & 0.634 & $79.6 \%$ \\
\hline 46067 & SD & Hutchinson & rural & 265 & $28.2 \%$ & 812.90 & 0.326 & $73.4 \%$ \\
\hline
\end{tabular}




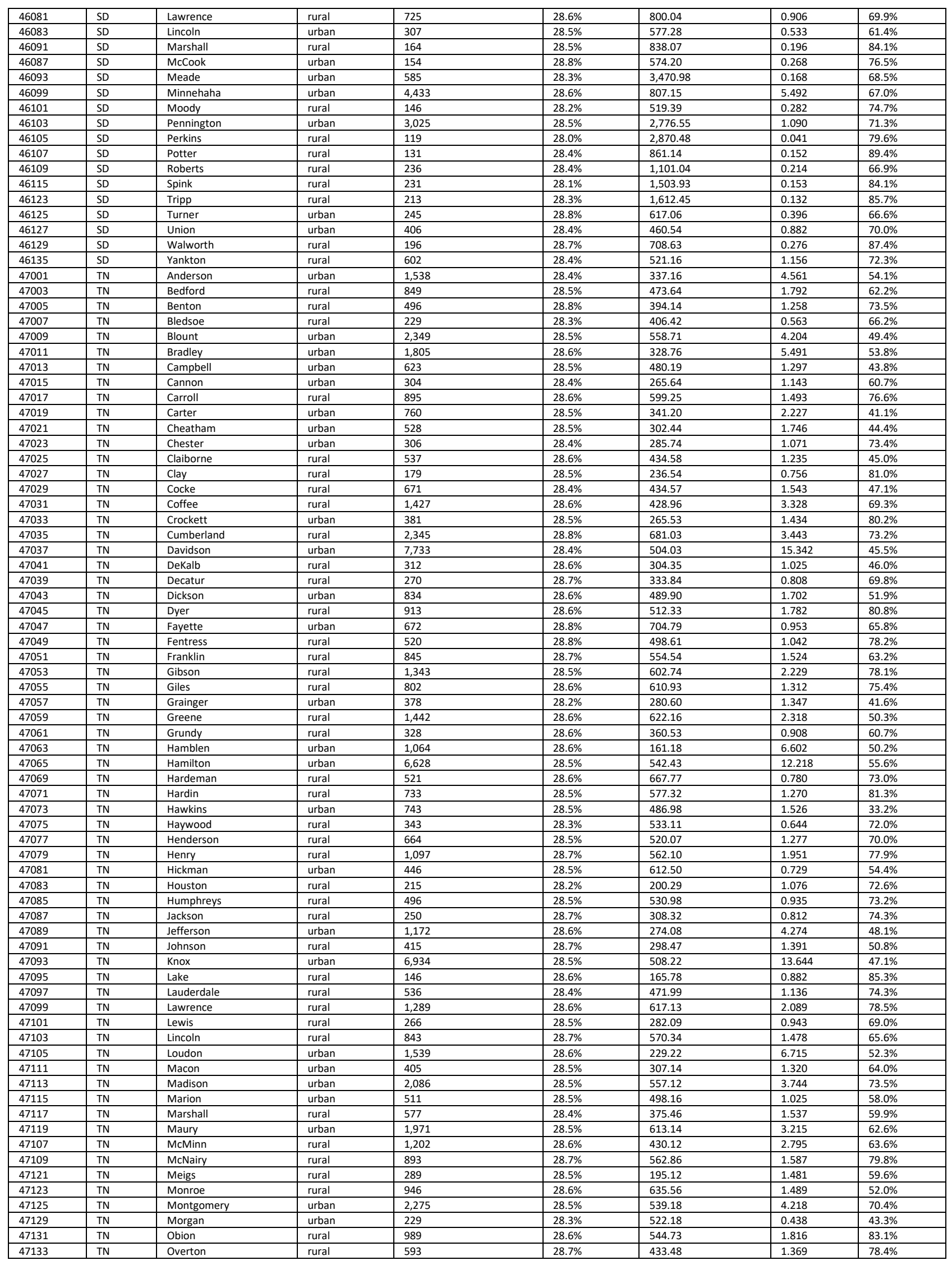




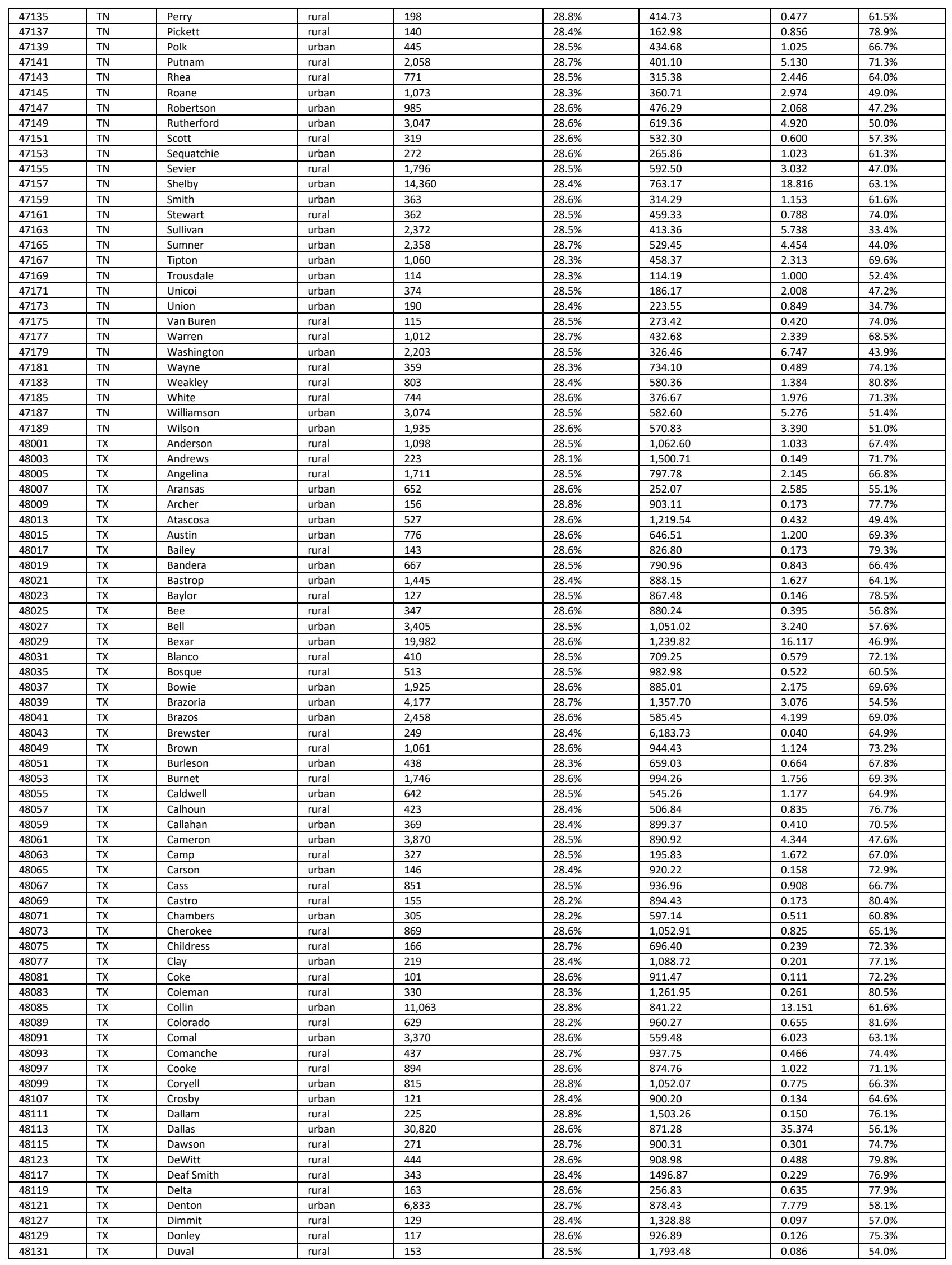




\begin{tabular}{|c|c|c|c|c|c|c|c|c|}
\hline 48133 & TX & Eastland & rural & 574 & $28.9 \%$ & 926.49 & 0.619 & $71.0 \%$ \\
\hline 48135 & TX & Ector & urban & 2,061 & $28.4 \%$ & 897.69 & 2.296 & $69.9 \%$ \\
\hline 48141 & $T X$ & El Paso & urban & 7,479 & $28.5 \%$ & 1012.69 & 7.385 & $41.0 \%$ \\
\hline 48139 & $\mathrm{TX}$ & Ellis & urban & 2,684 & $28.5 \%$ & 935.49 & 2.869 & $62.4 \%$ \\
\hline 48143 & TX & Erath & rural & 757 & $28.5 \%$ & $1,083.07$ & 0.699 & $69.6 \%$ \\
\hline 48145 & $T X$ & Falls & urban & 235 & $27.9 \%$ & 765.48 & 0.307 & $56.8 \%$ \\
\hline 48147 & $\mathrm{TX}$ & Fannin & rural & 836 & $28.7 \%$ & 890.84 & 0.939 & $72.5 \%$ \\
\hline 48149 & $T X$ & Fayette & rural & 933 & $28.4 \%$ & 950.01 & 0.983 & $78.0 \%$ \\
\hline 48151 & $T X$ & Fisher & rural & 113 & $28.8 \%$ & 898.94 & 0.126 & $76.2 \%$ \\
\hline 48153 & $\mathrm{TX}$ & Floyd & rural & 138 & $28.8 \%$ & 992.14 & 0.139 & $69.9 \%$ \\
\hline 48159 & $\mathrm{TX}$ & Franklin & rural & 238 & $28.9 \%$ & 284.39 & 0.835 & $72.1 \%$ \\
\hline 48161 & $\mathrm{TX}$ & Freestone & rural & 426 & $28.6 \%$ & 877.74 & 0.485 & $71.3 \%$ \\
\hline 48163 & $\mathrm{TX}$ & Frio & rural & 182 & $28.7 \%$ & $1,133.50$ & 0.161 & $58.6 \%$ \\
\hline 48165 & $T X$ & Gaines & rural & 219 & $28.4 \%$ & $1,502.38$ & 0.146 & $78.4 \%$ \\
\hline 48167 & $T X$ & Galveston & urban & 5,228 & $28.4 \%$ & 378.36 & 13.818 & $60.4 \%$ \\
\hline 48171 & $T X$ & Gillespie & rural & 1,210 & $28.6 \%$ & $1,058.21$ & 1.143 & $77.4 \%$ \\
\hline 48175 & TX & Goliad & urban & 161 & $28.2 \%$ & 852.01 & 0.188 & $73.1 \%$ \\
\hline 48177 & $\mathrm{TX}$ & Gonzales & rural & 421 & $28.4 \%$ & $1,066.69$ & 0.394 & $72.7 \%$ \\
\hline 48179 & $T X$ & Gray & rural & 583 & $28.8 \%$ & 925.97 & 0.629 & $76.4 \%$ \\
\hline 48185 & $\mathrm{TX}$ & Grimes & rural & 552 & $28.2 \%$ & 787.46 & 0.700 & $66.3 \%$ \\
\hline 48187 & $\mathrm{TX}$ & Guadalupe & urban & 2,172 & $28.5 \%$ & 711.30 & 3.054 & $63.0 \%$ \\
\hline 48189 & $\mathrm{TX}$ & Hale & rural & 665 & $28.5 \%$ & $1,004.68$ & 0.662 & $71.9 \%$ \\
\hline 48191 & $\mathrm{TX}$ & Hall & rural & 106 & $29.0 \%$ & 883.49 & 0.120 & $77.7 \%$ \\
\hline 48193 & $T X$ & Hamilton & rural & 229 & $28.8 \%$ & 835.91 & 0.274 & $64.1 \%$ \\
\hline 48195 & $\mathrm{TX}$ & Hansford & rural & 145 & $28.4 \%$ & 919.81 & 0.157 & $81.7 \%$ \\
\hline 48197 & $\mathrm{TX}$ & Hardeman & rural & 115 & $28.3 \%$ & 695.11 & 0.165 & $75.9 \%$ \\
\hline 48199 & $\mathrm{TX}$ & Hardin & urban & 918 & $28.7 \%$ & 890.57 & 1.031 & $53.2 \%$ \\
\hline 48201 & $\mathrm{TX}$ & Harris & urban & 41,061 & $28.6 \%$ & $1,703.48$ & 24.104 & $47.1 \%$ \\
\hline 48203 & $T X$ & Harrison & rural & 1,067 & $28.1 \%$ & 899.95 & 1.186 & $67.9 \%$ \\
\hline 48207 & $\mathrm{TX}$ & Haskell & rural & 183 & $28.7 \%$ & 903.13 & 0.202 & $75.7 \%$ \\
\hline 48209 & $\mathrm{TX}$ & Hays & urban & 2,354 & $28.6 \%$ & 677.98 & 3.473 & $61.5 \%$ \\
\hline 48213 & $T X$ & Henderson & rural & 1,624 & $28.6 \%$ & 873.75 & 1.859 & $66.4 \%$ \\
\hline 48215 & $\mathrm{TX}$ & Hidalgo & urban & 6,085 & $28.6 \%$ & $1,570.87$ & 3.873 & $51.7 \%$ \\
\hline 48217 & $\mathrm{TX}$ & Hill & rural & 938 & $28.5 \%$ & 958.86 & 0.978 & $68.3 \%$ \\
\hline 48219 & $T X$ & Hockley & rural & 408 & $28.4 \%$ & 908.39 & 0.449 & $68.5 \%$ \\
\hline 48221 & TX & Hood & urban & 1,906 & $28.6 \%$ & 420.64 & 4.531 & $67.5 \%$ \\
\hline 48223 & $\mathrm{TX}$ & Hopkins & rural & 936 & $28.6 \%$ & 767.17 & 1.220 & $79.1 \%$ \\
\hline 48233 & TX & Hutchinson & rural & 526 & $28.5 \%$ & 887.42 & 0.593 & $77.9 \%$ \\
\hline 48237 & $T X$ & Jack & rural & 192 & $28.5 \%$ & 910.66 & 0.211 & $76.9 \%$ \\
\hline 48239 & TX & Jackson & rural & 253 & $28.5 \%$ & 829.43 & 0.305 & $70.5 \%$ \\
\hline 48241 & $\mathrm{TX}$ & Jasper & rural & 702 & $28.5 \%$ & 938.85 & 0.748 & $63.5 \%$ \\
\hline 48245 & $T X$ & Jefferson & urban & 3,415 & $28.4 \%$ & 876.30 & 3.897 & $53.7 \%$ \\
\hline 48249 & $\mathrm{TX}$ & Jim Wells & rural & 493 & $28.5 \%$ & 864.97 & 0.569 & $46.4 \%$ \\
\hline 48251 & TX & Johnson & urban & 2,394 & $28.7 \%$ & 724.69 & 3.304 & $47.2 \%$ \\
\hline 48253 & TX & Jones & urban & 338 & $28.4 \%$ & 928.55 & 0.364 & $73.2 \%$ \\
\hline 48255 & TX & Karnes & rural & 310 & $28.1 \%$ & 747.56 & 0.414 & $73.6 \%$ \\
\hline 48257 & TX & Kaufman & urban & 2,186 & $28.5 \%$ & 780.70 & 2.799 & $61.9 \%$ \\
\hline 48259 & $T X$ & Kendall & urban & 1,484 & $28.8 \%$ & 662.45 & 2.240 & $66.6 \%$ \\
\hline 48265 & $\mathrm{TX}$ & Kerr & rural & 2,265 & $28.8 \%$ & $1,103.32$ & 2.053 & $78.0 \%$ \\
\hline 48267 & $\mathrm{TX}$ & Kimble & rural & 162 & $28.9 \%$ & $1,250.99$ & 0.130 & $77.0 \%$ \\
\hline 48273 & TX & Kleberg & rural & 319 & $28.7 \%$ & 881.31 & 0.362 & $48.1 \%$ \\
\hline 48277 & $T X$ & Lamar & rural & 1,480 & $28.5 \%$ & 907.19 & 1.632 & $83.8 \%$ \\
\hline 48279 & $T X$ & Lamb & rural & 304 & $28.4 \%$ & $1,016.18$ & 0.299 & $71.7 \%$ \\
\hline 48281 & $T X$ & Lampasas & urban & 505 & $28.7 \%$ & 712.84 & 0.708 & $65.4 \%$ \\
\hline 48285 & $T X$ & Lavaca & rural & 741 & $28.4 \%$ & 969.71 & 0.764 & $79.0 \%$ \\
\hline 48287 & $T X$ & Lee & rural & 353 & $28.3 \%$ & 629.02 & 0.562 & $67.2 \%$ \\
\hline 48289 & $\mathrm{TX}$ & Leon & rural & 601 & $28.5 \%$ & $1,073.15$ & 0.560 & $68.6 \%$ \\
\hline 48291 & $T X$ & Liberty & urban & 1,120 & $28.3 \%$ & $1,158.42$ & 0.967 & $50.6 \%$ \\
\hline 48293 & $T X$ & Limestone & rural & 448 & $28.4 \%$ & 905.29 & 0.495 & $64.5 \%$ \\
\hline 48297 & $T X$ & Live Oak & rural & 167 & $28.7 \%$ & 1039.7 & 0.161 & $63.4 \%$ \\
\hline 48299 & $T X$ & Llano & rural & 786 & $28.8 \%$ & 934.03 & 0.842 & $70.3 \%$ \\
\hline 48303 & $\mathrm{TX}$ & Lubbock & urban & 4,319 & $28.6 \%$ & 895.60 & 4.822 & $60.8 \%$ \\
\hline 48305 & $\mathrm{TX}$ & Lynn & urban & 134 & $28.9 \%$ & 891.87 & 0.150 & $72.8 \%$ \\
\hline 48313 & $\mathrm{TX}$ & Madison & rural & 252 & $28.8 \%$ & 466.07 & 0.541 & $67.8 \%$ \\
\hline 48315 & $\mathrm{TX}$ & Marion & rural & 254 & $28.4 \%$ & 380.88 & 0.667 & $69.4 \%$ \\
\hline 48319 & $T X$ & Mason & rural & 169 & $28.6 \%$ & 928.80 & 0.182 & $75.4 \%$ \\
\hline 48321 & $T X$ & Matagorda & rural & 632 & $28.5 \%$ & $1,100.28$ & 0.575 & $65.4 \%$ \\
\hline 48323 & $\mathrm{TX}$ & Maverick & rural & 697 & $27.9 \%$ & $1,279.26$ & 0.545 & $55.5 \%$ \\
\hline 48307 & $\mathrm{TX}$ & McCulloch & rural & 264 & $28.6 \%$ & $1,065.60$ & 0.247 & $79.5 \%$ \\
\hline 48309 & $\mathrm{TX}$ & McLennan & urban & 4,020 & $28.5 \%$ & $1,037.10$ & 3.876 & $59.2 \%$ \\
\hline 48325 & $T X$ & Medina & urban & 691 & $28.6 \%$ & $1,325.36$ & 0.521 & $55.4 \%$ \\
\hline 48329 & $T X$ & Midland & urban & 2,109 & $28.3 \%$ & 900.30 & 2.342 & $70.7 \%$ \\
\hline 48331 & $T X$ & Milam & rural & 489 & $28.1 \%$ & $1,016.93$ & 0.481 & $56.6 \%$ \\
\hline 48333 & $T X$ & Mills & rural & 139 & $28.1 \%$ & 748.26 & 0.185 & $63.8 \%$ \\
\hline
\end{tabular}




\begin{tabular}{|c|c|c|c|c|c|c|c|c|}
\hline 48339 & TX & Montgomery & urban & 7,015 & $28.6 \%$ & $1,041.73$ & 6.734 & $52.4 \%$ \\
\hline 48341 & TX & Moore & rural & 316 & $28.4 \%$ & 899.69 & 0.351 & $76.1 \%$ \\
\hline 48343 & $T X$ & Morris & rural & 330 & $28.3 \%$ & 251.98 & 1.309 & $61.9 \%$ \\
\hline 48347 & $\mathrm{TX}$ & Nacogdoches & rural & 1,214 & $28.5 \%$ & 946.54 & 1.283 & $71.5 \%$ \\
\hline 48349 & $\mathrm{TX}$ & Navarro & rural & 1,023 & $28.5 \%$ & $1,009.63$ & 1.013 & $71.0 \%$ \\
\hline 48351 & $T X$ & Newton & urban & 238 & $28.4 \%$ & 933.68 & 0.255 & $65.5 \%$ \\
\hline 48353 & $\mathrm{TX}$ & Nolan & rural & 377 & $28.3 \%$ & 912.00 & 0.413 & $74.2 \%$ \\
\hline 48355 & $T X$ & Nueces & urban & 3,440 & $28.7 \%$ & 838.48 & 4.103 & $39.5 \%$ \\
\hline 48357 & $T X$ & Ochiltree & rural & 197 & $28.8 \%$ & 917.63 & 0.215 & $84.3 \%$ \\
\hline 48361 & $\mathrm{TX}$ & Orange & urban & 1,407 & $28.4 \%$ & 333.67 & 4.218 & $55.7 \%$ \\
\hline 48365 & $\mathrm{TX}$ & Panola & rural & 516 & $28.5 \%$ & 801.75 & 0.644 & $71.5 \%$ \\
\hline 48367 & $\mathrm{TX}$ & Parker & urban & 2,306 & $28.6 \%$ & 903.48 & 2.552 & $66.7 \%$ \\
\hline 48369 & $\mathrm{TX}$ & Parmer & rural & 207 & $28.5 \%$ & 880.78 & 0.235 & $84.8 \%$ \\
\hline 48371 & $T X$ & Pecos & rural & 228 & $28.1 \%$ & $4,763.85$ & 0.048 & $68.2 \%$ \\
\hline 48373 & $T X$ & Polk & rural & 2,038 & $28.6 \%$ & $1,057.09$ & 1.928 & $66.8 \%$ \\
\hline 48375 & $T X$ & Potter & urban & 4,118 & $28.6 \%$ & 908.37 & 4.534 & $68.9 \%$ \\
\hline 48377 & $T X$ & Presidio & rural & 195 & $27.1 \%$ & $3,855.24$ & 0.051 & $71.3 \%$ \\
\hline 48379 & $\mathrm{TX}$ & Rains & rural & 345 & $28.6 \%$ & 229.45 & 1.503 & $73.9 \%$ \\
\hline 48381 & $\mathrm{TX}$ & Randall & urban & 653 & $28.5 \%$ & 911.54 & 0.716 & $73.9 \%$ \\
\hline 48389 & $\mathrm{TX}$ & Reeves & rural & 208 & $27.9 \%$ & $2,635.37$ & 0.079 & $73.1 \%$ \\
\hline 48391 & $T X$ & Refugio & rural & 165 & $28.3 \%$ & 770.44 & 0.215 & $68.0 \%$ \\
\hline 48395 & $\mathrm{TX}$ & Robertson & urban & 337 & $28.4 \%$ & 855.68 & 0.393 & $68.4 \%$ \\
\hline 48397 & $\mathrm{TX}$ & Rockwall & urban & 1,494 & $28.7 \%$ & 127.04 & 11.759 & $64.9 \%$ \\
\hline 48399 & $T X$ & Runnels & rural & 367 & $28.7 \%$ & $1,050.94$ & 0.350 & $83.6 \%$ \\
\hline 48401 & $\mathrm{TX}$ & Rusk & urban & 860 & $28.4 \%$ & 924.03 & 0.930 & $67.8 \%$ \\
\hline 48403 & $\mathrm{TX}$ & Sabine & rural & 489 & $28.6 \%$ & 491.39 & 0.996 & $75.5 \%$ \\
\hline 48405 & $\mathrm{TX}$ & San Augustine & rural & 233 & $28.2 \%$ & 530.66 & 0.440 & $72.6 \%$ \\
\hline 48407 & $\mathrm{TX}$ & San Jacinto & rural & 354 & $28.6 \%$ & 569.24 & 0.621 & $52.7 \%$ \\
\hline 48409 & $\mathrm{TX}$ & San Patricio & urban & 859 & $28.8 \%$ & 693.45 & 1.239 & $41.0 \%$ \\
\hline 48411 & $\mathrm{TX}$ & San Saba & rural & 176 & $28.4 \%$ & 1135.3 & 0.155 & $68.8 \%$ \\
\hline 48415 & $\mathrm{TX}$ & Scurry & rural & 354 & $28.5 \%$ & 905.44 & 0.391 & $72.4 \%$ \\
\hline 48419 & $\mathrm{TX}$ & Shelby & rural & 580 & $28.3 \%$ & 795.58 & 0.729 & $72.9 \%$ \\
\hline 48423 & $\mathrm{TX}$ & Smith & urban & 4,971 & $28.5 \%$ & 921.45 & 5.395 & $70.2 \%$ \\
\hline 48425 & $\mathrm{TX}$ & Somervell & urban & 175 & $28.1 \%$ & 186.46 & 0.941 & $65.0 \%$ \\
\hline 48427 & $T X$ & Starr & rural & 696 & $27.8 \%$ & $1,223.18$ & 0.569 & $67.6 \%$ \\
\hline 48429 & TX & Stephens & rural & 223 & $28.4 \%$ & 896.72 & 0.248 & $73.0 \%$ \\
\hline 48435 & $\mathrm{TX}$ & Sutton & rural & 102 & $28.8 \%$ & $1,453.93$ & 0.070 & $86.5 \%$ \\
\hline 48445 & TX & Terry & rural & 209 & $28.6 \%$ & 888.84 & 0.235 & $64.3 \%$ \\
\hline 48449 & TX & Titus & rural & 571 & $28.5 \%$ & 406.05 & 1.407 & $69.6 \%$ \\
\hline 48451 & TX & Tom Green & urban & 2,315 & $28.5 \%$ & 1521.97 & 1.521 & $71.2 \%$ \\
\hline 48453 & $\mathrm{TX}$ & Travis & urban & 12,967 & $28.5 \%$ & 990.20 & 13.096 & $61.8 \%$ \\
\hline 48455 & $T X$ & Trinity & rural & 419 & $28.5 \%$ & 693.61 & 0.604 & $65.4 \%$ \\
\hline 48457 & $\mathrm{TX}$ & Tyler & rural & 500 & $28.6 \%$ & 924.50 & 0.541 & $68.2 \%$ \\
\hline 48459 & TX & Upshur & urban & 788 & $28.4 \%$ & 582.95 & 1.351 & $65.0 \%$ \\
\hline 48463 & TX & Uvalde & rural & 503 & $28.5 \%$ & $1,551.95$ & 0.324 & $63.8 \%$ \\
\hline 48465 & TX & Val Verde & rural & 733 & $28.0 \%$ & 3144.75 & 0.233 & $63.8 \%$ \\
\hline 48467 & TX & Van Zandt & rural & 1,366 & $28.6 \%$ & 842.56 & 1.621 & $69.1 \%$ \\
\hline 48469 & $T X$ & Victoria & urban & 1,589 & $28.6 \%$ & 882.14 & 1.802 & $67.2 \%$ \\
\hline 48471 & $\mathrm{TX}$ & Walker & rural & 915 & $28.6 \%$ & 784.17 & 1.167 & $58.8 \%$ \\
\hline 48473 & $\mathrm{TX}$ & Waller & urban & 478 & $28.2 \%$ & 513.43 & 0.930 & $56.7 \%$ \\
\hline 48475 & TX & Ward & rural & 162 & $28.5 \%$ & 835.60 & 0.194 & $68.4 \%$ \\
\hline 48477 & $T X$ & Washington & rural & 1,079 & $28.4 \%$ & 603.95 & 1.786 & $74.8 \%$ \\
\hline 48479 & $T X$ & Webb & urban & 2,453 & $28.5 \%$ & $3,361.48$ & 0.730 & $62.0 \%$ \\
\hline 48481 & $T X$ & Wharton & rural & 920 & $28.3 \%$ & $1,086.15$ & 0.847 & $74.4 \%$ \\
\hline 48483 & $T X$ & Wheeler & rural & 168 & $28.6 \%$ & 914.52 & 0.184 & $80.4 \%$ \\
\hline 48485 & $T X$ & Wichita & urban & 2,746 & $28.5 \%$ & 627.78 & 4.374 & $77.7 \%$ \\
\hline 48487 & $\mathrm{TX}$ & Wilbarger & rural & 270 & $28.3 \%$ & 970.84 & 0.278 & $67.1 \%$ \\
\hline 48489 & $T X$ & Willacy & rural & 200 & $28.8 \%$ & 590.55 & 0.338 & $50.0 \%$ \\
\hline 48491 & $T X$ & Williamson & urban & 7,463 & $28.8 \%$ & $1,118.30$ & 6.673 & $61.1 \%$ \\
\hline 48493 & $T X$ & Wilson & urban & 623 & $28.4 \%$ & 803.73 & 0.775 & $55.1 \%$ \\
\hline 48495 & $T X$ & Winkler & rural & 119 & $28.6 \%$ & 841.11 & 0.142 & $74.6 \%$ \\
\hline 48497 & $\mathrm{TX}$ & Wise & urban & 1,096 & $28.6 \%$ & 904.42 & 1.212 & $65.1 \%$ \\
\hline 48499 & $\mathrm{TX}$ & Wood & rural & 1,667 & $28.8 \%$ & 645.23 & 2.584 & $69.8 \%$ \\
\hline 48501 & $\mathrm{TX}$ & Yoakum & rural & 152 & $28.5 \%$ & 799.71 & 0.190 & $77.1 \%$ \\
\hline 48503 & $\mathrm{TX}$ & Young & rural & 605 & $28.5 \%$ & 914.47 & 0.661 & $87.3 \%$ \\
\hline 48505 & $T X$ & Zapata & rural & 174 & $28.3 \%$ & 998.41 & 0.174 & $74.2 \%$ \\
\hline 48507 & $T X$ & Zavala & rural & 125 & $28.6 \%$ & $1,297.41$ & 0.096 & $53.9 \%$ \\
\hline 49001 & UT & Beaver & rural & 175 & $28.7 \%$ & $2,589.88$ & 0.068 & $88.3 \%$ \\
\hline 49003 & UT & Box Elder & urban & 745 & $28.6 \%$ & 5745.55 & 0.130 & $54.4 \%$ \\
\hline 49005 & UT & Cache & urban & 1,033 & $28.6 \%$ & $1,164.81$ & 0.887 & $43.1 \%$ \\
\hline 49007 & UT & Carbon & rural & 552 & $28.4 \%$ & $1,478.49$ & 0.373 & $86.9 \%$ \\
\hline 49011 & UT & Davis & urban & 3,342 & $28.6 \%$ & 298.78 & 11.184 & $51.0 \%$ \\
\hline 49013 & UT & Duchesne & rural & 333 & $28.5 \%$ & $3,240.94$ & 0.103 & $65.1 \%$ \\
\hline 49015 & UT & Emery & rural & 289 & $28.5 \%$ & $4,462.31$ & 0.065 & $89.7 \%$ \\
\hline
\end{tabular}




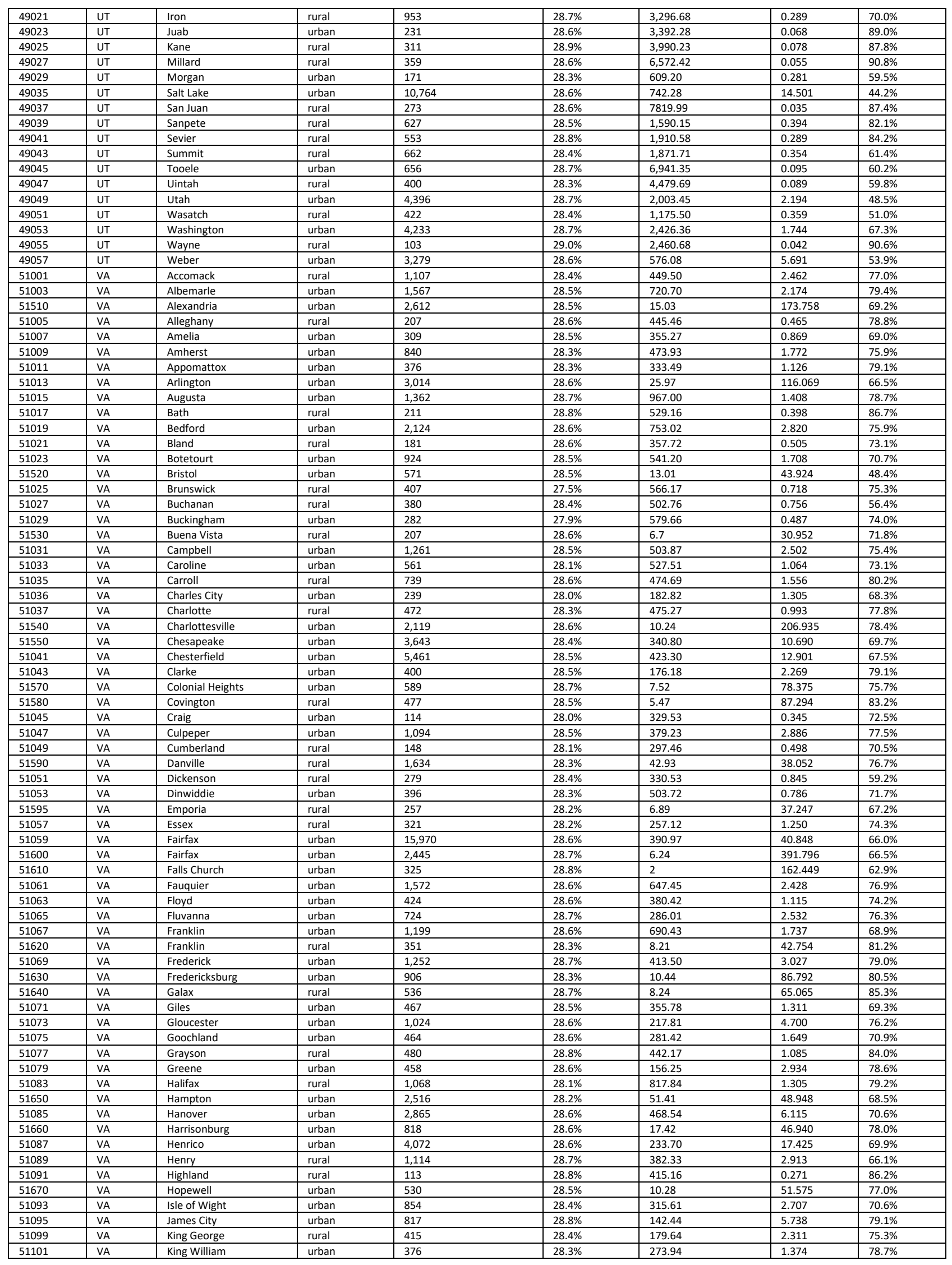




\begin{tabular}{|c|c|c|c|c|c|c|c|c|}
\hline 51097 & VA & King and Queen & rural & 190 & $28.2 \%$ & 315.14 & 0.603 & $77.6 \%$ \\
\hline 51103 & VA & Lancaster & rural & 724 & $28.6 \%$ & 133.25 & 5.432 & $82.8 \%$ \\
\hline 51105 & VA & Lee & rural & 431 & $28.7 \%$ & 435.52 & 0.990 & $55.4 \%$ \\
\hline 51678 & VA & Lexington & rural & 583 & $28.6 \%$ & 2.50 & 233.150 & $81.6 \%$ \\
\hline 51107 & VA & Loudoun & urban & 4,172 & $28.7 \%$ & 515.56 & 8.092 & $68.0 \%$ \\
\hline 51109 & VA & Louisa & rural & 933 & $28.5 \%$ & 496.30 & 1.879 & $75.6 \%$ \\
\hline 51111 & VA & Lunenburg & rural & 266 & $28.2 \%$ & 431.68 & 0.616 & $77.9 \%$ \\
\hline 51680 & VA & Lynchburg & urban & 2,148 & $28.4 \%$ & 49.13 & 43.730 & $76.8 \%$ \\
\hline 51113 & VA & Madison & rural & 338 & $28.5 \%$ & 320.68 & 1.055 & $79.2 \%$ \\
\hline 51683 & VA & Manassas & urban & 1,876 & $28.7 \%$ & 9.88 & 189.897 & $66.1 \%$ \\
\hline 51115 & VA & Mathews & urban & 428 & $28.5 \%$ & 85.93 & 4.982 & $81.5 \%$ \\
\hline 51117 & VA & Mecklenburg & rural & 1,045 & $28.4 \%$ & 625.49 & 1.671 & $75.9 \%$ \\
\hline 51119 & VA & Middlesex & rural & 518 & $28.6 \%$ & 130.31 & 3.972 & $78.7 \%$ \\
\hline 51121 & VA & Montgomery & urban & 1,662 & $28.6 \%$ & 387.01 & 4.293 & $74.4 \%$ \\
\hline 51125 & VA & Nelson & urban & 685 & $28.4 \%$ & 470.86 & 1.455 & $79.2 \%$ \\
\hline 51127 & VA & New Kent & urban & 454 & $28.3 \%$ & 209.73 & 2.163 & $74.3 \%$ \\
\hline 51700 & VA & Newport News & urban & 2,963 & $28.4 \%$ & 68.71 & 43.124 & $68.9 \%$ \\
\hline 51710 & VA & Norfolk & urban & 2,970 & $28.2 \%$ & 54.12 & 54.869 & $65.8 \%$ \\
\hline 51131 & VA & Northampton & rural & 423 & $28.2 \%$ & 211.61 & 2.001 & $76.7 \%$ \\
\hline 51720 & VA & Norton & rural & 114 & $28.6 \%$ & 7.48 & 15.198 & $58.9 \%$ \\
\hline 51135 & VA & Nottoway & rural & 386 & $28.2 \%$ & 314.39 & 1.228 & $74.8 \%$ \\
\hline 51137 & VA & Orange & rural & 1,295 & $28.6 \%$ & 340.78 & 3.801 & $79.1 \%$ \\
\hline 51139 & VA & Page & rural & 697 & $28.7 \%$ & 310.86 & 2.241 & $80.5 \%$ \\
\hline 51141 & VA & Patrick & rural & 583 & $28.6 \%$ & 483.10 & 1.208 & $69.9 \%$ \\
\hline 51730 & VA & Petersburg & urban & 755 & $28.1 \%$ & 22.93 & 32.908 & $69.2 \%$ \\
\hline 51143 & VA & Pittsylvania & rural & 1,254 & $28.4 \%$ & 968.94 & 1.294 & $75.1 \%$ \\
\hline 51735 & VA & Poquoson & urban & 244 & $28.6 \%$ & 15.32 & 15.911 & $80.5 \%$ \\
\hline 51740 & VA & Portsmouth & urban & 1,497 & $28.0 \%$ & 33.65 & 44.500 & $64.3 \%$ \\
\hline 51145 & VA & Powhatan & urban & 736 & $28.4 \%$ & 260.22 & 2.830 & $70.6 \%$ \\
\hline 51147 & VA & Prince Edward & rural & 575 & $28.0 \%$ & 349.96 & 1.644 & $75.6 \%$ \\
\hline 51149 & VA & Prince George & urban & 436 & $28.4 \%$ & 265.16 & 1.644 & $78.9 \%$ \\
\hline 51153 & VA & Prince William & urban & 3,860 & $28.8 \%$ & 336.4 & 11.473 & $67.4 \%$ \\
\hline 51155 & VA & Pulaski & urban & 853 & $28.6 \%$ & 319.86 & 2.666 & $75.3 \%$ \\
\hline 51750 & VA & Radford & urban & 396 & $28.9 \%$ & 9.87 & 40.135 & $74.0 \%$ \\
\hline 51157 & VA & Rappahannock & urban & 390 & $28.5 \%$ & 266.23 & 1.466 & $80.5 \%$ \\
\hline 51159 & VA & Richmond & rural & 229 & $28.5 \%$ & 191.49 & 1.198 & $74.1 \%$ \\
\hline 51760 & VA & Richmond & urban & 5,838 & $28.4 \%$ & 59.81 & 97.604 & $61.0 \%$ \\
\hline 51161 & VA & Roanoke & urban & 1,281 & $28.6 \%$ & 250.52 & 5.113 & $76.2 \%$ \\
\hline 51167 & VA & Russell & rural & 560 & $28.5 \%$ & 473.82 & 1.182 & $63.6 \%$ \\
\hline 51775 & VA & Salem & urban & 1,078 & $28.5 \%$ & 14.44 & 74.679 & $74.4 \%$ \\
\hline 51169 & VA & Scott & urban & 388 & $28.3 \%$ & 535.53 & 0.725 & $38.7 \%$ \\
\hline 51171 & VA & Shenandoah & rural & 1,396 & $28.8 \%$ & 508.78 & 2.744 & $80.1 \%$ \\
\hline 51173 & VA & Smyth & rural & 856 & $28.5 \%$ & 450.93 & 1.899 & $71.5 \%$ \\
\hline 51175 & VA & Southampton & rural & 315 & $28.2 \%$ & 599.14 & 0.526 & $76.5 \%$ \\
\hline 51177 & VA & Spotsylvania & urban & 1,427 & $28.7 \%$ & 401.50 & 3.555 & $76.2 \%$ \\
\hline 51179 & VA & Stafford & urban & 2,326 & $28.8 \%$ & 268.96 & 8.647 & $75.9 \%$ \\
\hline 51790 & VA & Staunton & urban & 1,215 & $28.6 \%$ & 19.98 & 60.793 & $82.2 \%$ \\
\hline 51800 & VA & Suffolk & urban & 1,595 & $28.4 \%$ & 400.17 & 3.985 & $72.1 \%$ \\
\hline 51181 & VA & Surry & rural & 146 & $28.5 \%$ & 278.95 & 0.524 & $65.9 \%$ \\
\hline 51183 & VA & Sussex & urban & 272 & $28.2 \%$ & 490.22 & 0.554 & $70.7 \%$ \\
\hline 51185 & VA & Tazewell & rural & 1,001 & $28.5 \%$ & 518.85 & 1.928 & $64.8 \%$ \\
\hline 51810 & VA & Virginia Beach & urban & 8,215 & $28.6 \%$ & 249.02 & 32.987 & $74.3 \%$ \\
\hline 51187 & VA & Warren & urban & 892 & $28.5 \%$ & 213.47 & 4.177 & $80.5 \%$ \\
\hline 51191 & VA & Washington & urban & 1,153 & $28.6 \%$ & 560.97 & 2.055 & $62.8 \%$ \\
\hline 51820 & VA & Waynesboro & urban & 945 & $28.7 \%$ & 15.04 & 62.831 & $79.3 \%$ \\
\hline 51193 & VA & Westmoreland & rural & 590 & $28.5 \%$ & 229.37 & 2.572 & $79.0 \%$ \\
\hline 51830 & VA & Williamsburg & urban & 1,128 & $28.6 \%$ & 9.02 & 125.110 & $79.4 \%$ \\
\hline 51840 & VA & Winchester & urban & 1,513 & $28.7 \%$ & 9.23 & 163.899 & $78.2 \%$ \\
\hline 51195 & VA & Wise & rural & 702 & $28.5 \%$ & 403.19 & 1.742 & $57.0 \%$ \\
\hline 51197 & VA & Wythe & rural & 929 & $28.6 \%$ & 461.82 & 2.012 & $75.2 \%$ \\
\hline 51199 & VA & York & urban & 2,965 & $28.7 \%$ & 104.78 & 28.298 & $79.8 \%$ \\
\hline 50001 & VT & Addison & rural & 1,063 & $28.5 \%$ & 766.33 & 1.387 & $78.2 \%$ \\
\hline 50003 & VT & Bennington & rural & 1,359 & $28.7 \%$ & 674.98 & 2.013 & $83.6 \%$ \\
\hline 50005 & VT & Caledonia & rural & 1,010 & $28.6 \%$ & 648.86 & 1.556 & $83.1 \%$ \\
\hline 50007 & VT & Chittenden & urban & 3,109 & $28.6 \%$ & 536.58 & 5.794 & $80.3 \%$ \\
\hline 50009 & VT & Essex & rural & 266 & $28.3 \%$ & 663.60 & 0.402 & $86.0 \%$ \\
\hline 50011 & VT & Franklin & urban & 1,128 & $28.6 \%$ & 633.71 & 1.780 & $81.0 \%$ \\
\hline 50013 & VT & Grand Isle & urban & 258 & $28.5 \%$ & 81.81 & 3.158 & $79.6 \%$ \\
\hline 50015 & VT & Lamoille & rural & 1,593 & $28.6 \%$ & 458.80 & 3.471 & $78.9 \%$ \\
\hline 50017 & VT & Orange & rural & 934 & $28.6 \%$ & 687.03 & 1.359 & $82.6 \%$ \\
\hline 50019 & VT & Orleans & rural & 990 & $28.6 \%$ & 693.27 & 1.428 & $83.3 \%$ \\
\hline 50021 & VT & Rutland & rural & 2,256 & $28.5 \%$ & 929.82 & 2.426 & $84.2 \%$ \\
\hline 50023 & VT & Washington & rural & 1,937 & $28.6 \%$ & 687.23 & 2.819 & $81.9 \%$ \\
\hline 50025 & VT & Windham & rural & 1,472 & $28.7 \%$ & 785.31 & 1.875 & $82.5 \%$ \\
\hline 50027 & VT & Windsor & rural & 2,147 & $28.6 \%$ & 969.34 & 2.215 & $81.1 \%$ \\
\hline
\end{tabular}




\begin{tabular}{|c|c|c|c|c|c|c|c|c|}
\hline 53005 & WA & Benton & urban & 4,067 & $28.6 \%$ & $1,700.38$ & 2.392 & $79.3 \%$ \\
\hline 53007 & WA & Chelan & urban & 1,575 & $28.5 \%$ & $2,920.52$ & 0.539 & $71.7 \%$ \\
\hline 53009 & WA & Clallam & rural & 3,995 & $28.7 \%$ & $1,738.33$ & 2.298 & $86.6 \%$ \\
\hline 53011 & WA & Clark & urban & 4,811 & $28.5 \%$ & 629.00 & 7.649 & $29.8 \%$ \\
\hline 53013 & WA & Columbia & urban & 171 & $28.8 \%$ & 868.63 & 0.197 & $89.3 \%$ \\
\hline 53015 & WA & Cowlitz & urban & 1,697 & $28.6 \%$ & $1,140.13$ & 1.489 & $41.1 \%$ \\
\hline 53017 & WA & Douglas & urban & 1,536 & $28.5 \%$ & $1,819.26$ & 0.844 & $69.0 \%$ \\
\hline 53019 & WA & Ferry & rural & 276 & $28.8 \%$ & $2,203.16$ & 0.125 & $87.1 \%$ \\
\hline 53021 & WA & Franklin & urban & 1,108 & $28.6 \%$ & $1,242.17$ & 0.892 & $78.1 \%$ \\
\hline 53023 & WA & Garfield & rural & 105 & $28.3 \%$ & 710.69 & 0.148 & $92.7 \%$ \\
\hline 53027 & WA & Grays Harbor & rural & 2,424 & $28.5 \%$ & 1902.03 & 1.274 & $84.6 \%$ \\
\hline 53029 & WA & Island & rural & 2,673 & $28.8 \%$ & 208.45 & 12.825 & $61.4 \%$ \\
\hline 53031 & WA & Jefferson & rural & 2,039 & $28.7 \%$ & $1,803.70$ & 1.130 & $87.9 \%$ \\
\hline 53033 & WA & King & urban & 30,846 & $28.5 \%$ & $2,115.57$ & 14.580 & $50.6 \%$ \\
\hline 53035 & WA & Kitsap & urban & 6,346 & $28.6 \%$ & 394.94 & 16.068 & $67.0 \%$ \\
\hline 53037 & WA & Kittitas & rural & 1,165 & $28.6 \%$ & $2,297.27$ & 0.507 & $84.9 \%$ \\
\hline 53039 & WA & Klickitat & rural & 853 & $28.4 \%$ & $1,871.31$ & 0.456 & $85.7 \%$ \\
\hline 53041 & WA & Lewis & rural & 1,975 & $28.6 \%$ & $2,402.80$ & 0.822 & $58.1 \%$ \\
\hline 53043 & WA & Lincoln & rural & 476 & $28.6 \%$ & $2,310.49$ & 0.206 & $90.5 \%$ \\
\hline 53047 & WA & Okanogan & rural & 1,488 & $28.4 \%$ & $5,267.98$ & 0.282 & $82.9 \%$ \\
\hline 53049 & WA & Pacific & rural & 1,063 & $28.8 \%$ & 932.66 & 1.140 & $90.1 \%$ \\
\hline 53051 & WA & Pend Oreille & urban & 512 & $28.7 \%$ & 1399.99 & 0.365 & $81.7 \%$ \\
\hline 53053 & WA & Pierce & urban & 14,131 & $28.5 \%$ & $1,669.51$ & 8.464 & $58.6 \%$ \\
\hline 53055 & WA & San Juan & rural & 829 & $28.6 \%$ & 173.91 & 4.768 & $69.8 \%$ \\
\hline 53057 & WA & Skagit & urban & 3,295 & $28.7 \%$ & $1,731.20$ & 1.903 & $59.9 \%$ \\
\hline 53059 & WA & Skamania & urban & 267 & $28.6 \%$ & $1,655.68$ & 0.162 & $78.5 \%$ \\
\hline 53061 & WA & Snohomish & urban & 8,550 & $28.6 \%$ & $2,087.27$ & 4.096 & $41.0 \%$ \\
\hline 53063 & WA & Spokane & urban & 9,571 & $28.5 \%$ & $1,763.79$ & 5.426 & $55.8 \%$ \\
\hline 53065 & WA & Stevens & urban & 1,501 & $28.5 \%$ & $2,477.76$ & 0.606 & $81.4 \%$ \\
\hline 53067 & WA & Thurston & urban & 5,263 & $28.7 \%$ & 721.96 & 7.289 & $52.6 \%$ \\
\hline 53069 & WA & Wahkiakum & rural & 166 & $28.7 \%$ & 263.38 & 0.629 & $64.6 \%$ \\
\hline 53071 & WA & Walla Walla & urban & 1,723 & $28.4 \%$ & 1270.13 & 1.356 & $80.0 \%$ \\
\hline 53073 & WA & Whatcom & urban & 4,136 & $28.5 \%$ & $2,106.86$ & 1.963 & $52.3 \%$ \\
\hline 53075 & WA & Whitman & rural & 819 & $28.7 \%$ & $2,159.09$ & 0.379 & $85.4 \%$ \\
\hline 53077 & WA & Yakima & urban & 4,695 & $28.5 \%$ & $4,295.40$ & 1.093 & $71.9 \%$ \\
\hline 55001 & $\mathrm{WI}$ & Adams & rural & 404 & $28.8 \%$ & 645.65 & 0.626 & $67.8 \%$ \\
\hline 55003 & WI & Ashland & rural & 417 & $28.6 \%$ & $1,045.04$ & 0.399 & $56.5 \%$ \\
\hline 55005 & WI & Barron & rural & 1,129 & $28.4 \%$ & 862.71 & 1.308 & $53.6 \%$ \\
\hline 55013 & WI & Burnett & rural & 479 & $28.4 \%$ & 821.85 & 0.583 & $57.9 \%$ \\
\hline 55015 & WI & Calumet & urban & 250 & $28.2 \%$ & 318.24 & 0.785 & $24.8 \%$ \\
\hline 55017 & WI & Chippewa & urban & 1,349 & $28.4 \%$ & $1,008.37$ & 1.338 & $59.4 \%$ \\
\hline 55019 & WI & Clark & rural & 515 & $28.3 \%$ & $1,209.82$ & 0.426 & $40.2 \%$ \\
\hline 55021 & WI & Columbia & urban & 1,448 & $28.5 \%$ & 765.53 & 1.892 & $60.8 \%$ \\
\hline 55023 & WI & Crawford & rural & 398 & $28.6 \%$ & 570.66 & 0.698 & $57.1 \%$ \\
\hline 55025 & WI & Dane & urban & 9,409 & $28.6 \%$ & $1,197.24$ & 7.859 & $64.5 \%$ \\
\hline 55027 & WI & Dodge & rural & 1,342 & $28.6 \%$ & 875.62 & 1.532 & $57.4 \%$ \\
\hline 55029 & WI & Door & rural & 1,120 & $28.5 \%$ & 481.98 & 2.323 & $66.7 \%$ \\
\hline 55031 & WI & Douglas & urban & 839 & $28.3 \%$ & $1,304.14$ & 0.643 & $49.2 \%$ \\
\hline 55033 & $\mathrm{WI}$ & Dunn & rural & 747 & $28.4 \%$ & 850.11 & 0.879 & $56.2 \%$ \\
\hline 55035 & WI & Eau Claire & urban & 2,105 & $28.5 \%$ & 637.98 & 3.299 & $61.1 \%$ \\
\hline 55037 & WI & Florence & rural & 107 & $28.1 \%$ & 488.20 & 0.220 & $63.1 \%$ \\
\hline 55039 & WI & Fond du Lac & urban & 1,649 & $28.5 \%$ & 719.55 & 2.291 & $40.7 \%$ \\
\hline 55041 & $\mathrm{WI}$ & Forest & rural & 257 & $28.7 \%$ & $1,014.07$ & 0.253 & $55.7 \%$ \\
\hline 55043 & $\mathrm{WI}$ & Grant & rural & 1,131 & $28.5 \%$ & $1,146.85$ & 0.987 & $53.9 \%$ \\
\hline 55045 & WI & Green & urban & 1,000 & $28.6 \%$ & 583.96 & 1.713 & $72.2 \%$ \\
\hline 55047 & $\mathrm{WI}$ & Green Lake & rural & 354 & $28.4 \%$ & 349.44 & 1.013 & $37.3 \%$ \\
\hline 55049 & WI & lowa & urban & 419 & $28.1 \%$ & 762.58 & 0.549 & $52.8 \%$ \\
\hline 55051 & WI & Iron & rural & 168 & $28.5 \%$ & 758.17 & 0.222 & $49.6 \%$ \\
\hline 55053 & WI & Jackson & rural & 344 & $28.4 \%$ & 987.72 & 0.348 & $47.7 \%$ \\
\hline 55055 & WI & Jefferson & rural & 1,761 & $28.6 \%$ & 556.47 & 3.164 & $59.8 \%$ \\
\hline 55057 & WI & Juneau & rural & 731 & $28.4 \%$ & 766.93 & 0.953 & $67.6 \%$ \\
\hline 55059 & WI & Kenosha & urban & 2,667 & $28.6 \%$ & 271.99 & 9.804 & $64.7 \%$ \\
\hline 55061 & WI & Kewaunee & urban & 359 & $28.4 \%$ & 342.52 & 1.049 & $42.2 \%$ \\
\hline 55063 & WI & La Crosse & urban & 1,782 & $28.4 \%$ & 451.69 & 3.946 & $42.9 \%$ \\
\hline 55065 & WI & Lafayette & rural & 349 & $28.3 \%$ & 633.59 & 0.552 & $61.0 \%$ \\
\hline 55067 & WI & Langlade & rural & 454 & $28.5 \%$ & 870.64 & 0.522 & $49.6 \%$ \\
\hline 55069 & WI & Lincoln & rural & 726 & $28.7 \%$ & 878.97 & 0.826 & $49.6 \%$ \\
\hline 55071 & WI & Manitowoc & rural & 1,530 & $28.5 \%$ & 589.08 & 2.597 & $46.4 \%$ \\
\hline 55073 & WI & Marathon & urban & 2,071 & $28.4 \%$ & $1,544.98$ & 1.340 & $44.0 \%$ \\
\hline 55075 & WI & Marinette & rural & 1,136 & $28.4 \%$ & $1,399.35$ & 0.812 & $52.6 \%$ \\
\hline 55077 & WI & Marquette & rural & 495 & $28.7 \%$ & 455.60 & 1.087 & $56.1 \%$ \\
\hline 55079 & WI & Milwaukee & urban & 11,235 & $28.3 \%$ & 241.40 & 46.542 & $47.1 \%$ \\
\hline 55081 & WI & Monroe & rural & 822 & $28.4 \%$ & 900.78 & 0.912 & $54.2 \%$ \\
\hline 55083 & WI & Oconto & urban & 566 & $28.4 \%$ & 997.99 & 0.567 & $38.6 \%$ \\
\hline 55085 & WI & Oneida & rural & 1,301 & $28.4 \%$ & $1,112.97$ & 1.169 & $58.9 \%$ \\
\hline
\end{tabular}




\begin{tabular}{|c|c|c|c|c|c|c|c|c|}
\hline 55091 & WI & Pepin & rural & 229 & $28.6 \%$ & 231.98 & 0.986 & $69.1 \%$ \\
\hline 55093 & $\overline{W I}$ & Pierce & urban & 719 & $28.3 \%$ & 573.75 & 1.253 & $49.4 \%$ \\
\hline 55095 & WI & Polk & rural & 824 & $28.4 \%$ & 913.96 & 0.902 & $49.5 \%$ \\
\hline 55097 & WI & Portage & rural & 1,143 & $28.5 \%$ & 800.68 & 1.428 & $49.6 \%$ \\
\hline 55099 & WI & Price & rural & 406 & $28.3 \%$ & $1,254.38$ & 0.324 & $53.4 \%$ \\
\hline 55101 & WI & Racine & urban & 3,611 & $28.6 \%$ & 332.50 & 10.861 & $53.5 \%$ \\
\hline 55103 & WI & Richland & rural & 472 & $28.5 \%$ & 586.15 & 0.805 & $72.6 \%$ \\
\hline 55105 & WI & Rock & urban & 3,042 & $28.6 \%$ & 718.14 & 4.236 & $58.0 \%$ \\
\hline 55107 & WI & Rusk & rural & 308 & $28.3 \%$ & 913.59 & 0.337 & $48.8 \%$ \\
\hline 55111 & WI & Sauk & rural & 1,222 & $28.6 \%$ & 830.90 & 1.470 & $52.0 \%$ \\
\hline 55113 & WI & Sawyer & rural & 494 & $28.5 \%$ & $1,257.30$ & 0.393 & $58.7 \%$ \\
\hline 55115 & WI & Shawano & rural & 596 & $28.5 \%$ & 893.06 & 0.667 & $34.0 \%$ \\
\hline 55117 & WI & Sheboygan & urban & 2,025 & $28.5 \%$ & 511.27 & 3.962 & $48.1 \%$ \\
\hline 55109 & WI & St. Croix & urban & 1,093 & $28.4 \%$ & 722.33 & 1.513 & $47.5 \%$ \\
\hline 55119 & $\mathrm{WI}$ & Taylor & rural & 327 & $28.3 \%$ & 974.88 & 0.335 & $46.7 \%$ \\
\hline 55121 & WI & Trempealeau & rural & 664 & $28.2 \%$ & 732.96 & 0.906 & $51.1 \%$ \\
\hline 55123 & WI & Vernon & rural & 489 & $28.4 \%$ & 791.58 & 0.618 & $36.9 \%$ \\
\hline 55125 & WI & Vilas & rural & 923 & $28.6 \%$ & 856.60 & 1.078 & $64.4 \%$ \\
\hline 55127 & WI & Walworth & rural & 2,395 & $28.6 \%$ & 555.13 & 4.315 & $70.5 \%$ \\
\hline 55129 & WI & Washburn & rural & 675 & $28.4 \%$ & 797.11 & 0.847 & $55.4 \%$ \\
\hline 55131 & WI & Washington & urban & 2,560 & $28.7 \%$ & 430.70 & 5.943 & $51.0 \%$ \\
\hline 55133 & WI & Waukesha & urban & 7,923 & $28.5 \%$ & 549.57 & 14.417 & $50.8 \%$ \\
\hline 55135 & WI & Waupaca & rural & 935 & $28.5 \%$ & 747.71 & 1.251 & $35.9 \%$ \\
\hline 55137 & WI & Waushara & rural & 425 & $28.4 \%$ & 626.15 & 0.679 & $39.9 \%$ \\
\hline 55139 & WI & Winnebago & urban & 1,920 & $28.5 \%$ & 434.49 & 4.420 & $34.0 \%$ \\
\hline 55141 & WI & Wood & rural & 1,473 & $28.5 \%$ & 793.12 & 1.857 & $38.6 \%$ \\
\hline 54001 & WV & Barbour & rural & 324 & $28.7 \%$ & 341.06 & 0.949 & $64.5 \%$ \\
\hline 54003 & WV & Berkeley & urban & 2,047 & $28.6 \%$ & 321.14 & 6.375 & $66.8 \%$ \\
\hline 54005 & WV & Boone & urban & 408 & $28.4 \%$ & 501.54 & 0.813 & $67.9 \%$ \\
\hline 54007 & WV & Braxton & rural & 280 & $28.3 \%$ & 510.81 & 0.549 & $59.7 \%$ \\
\hline 54009 & WV & Brooke & urban & 405 & $28.4 \%$ & 89.20 & 4.542 & $50.6 \%$ \\
\hline 54011 & WV & Cabell & urban & 2,043 & $28.5 \%$ & 281.02 & 7.269 & $55.8 \%$ \\
\hline 54013 & WV & Calhoun & rural & 183 & $28.4 \%$ & 279.25 & 0.655 & $69.0 \%$ \\
\hline 54015 & WV & Clay & urban & 199 & $28.5 \%$ & 341.90 & 0.582 & $57.2 \%$ \\
\hline 54017 & WV & Doddridge & rural & 112 & $28.8 \%$ & 319.72 & 0.349 & $60.8 \%$ \\
\hline 54019 & WV & Fayette & urban & 975 & $28.4 \%$ & 661.55 & 1.474 & $63.7 \%$ \\
\hline 54021 & WV & Gilmer & rural & 138 & $28.4 \%$ & 338.50 & 0.407 & $59.7 \%$ \\
\hline 54023 & WV & Grant & rural & 402 & $28.6 \%$ & 477.37 & 0.841 & $67.3 \%$ \\
\hline 54025 & WV & Greenbrier & rural & 950 & $28.5 \%$ & $1,019.57$ & 0.932 & $69.5 \%$ \\
\hline 54027 & WV & Hampshire & urban & 580 & $28.5 \%$ & 640.25 & 0.906 & $70.7 \%$ \\
\hline 54029 & WV & Hancock & urban & 880 & $28.5 \%$ & 82.61 & 10.651 & $66.3 \%$ \\
\hline 54031 & WV & Hardy & rural & 335 & $28.4 \%$ & 582.31 & 0.576 & $67.1 \%$ \\
\hline 54033 & WV & Harrison & rural & 1,549 & $28.5 \%$ & 416.01 & 3.723 & $61.5 \%$ \\
\hline 54035 & WV & Jackson & rural & 643 & $28.4 \%$ & 464.35 & 1.384 & $65.8 \%$ \\
\hline 54037 & WV & Jefferson & urban & 1,144 & $28.6 \%$ & 209.63 & 5.457 & $65.6 \%$ \\
\hline 54039 & WV & Kanawha & urban & 3,733 & $28.4 \%$ & 901.59 & 4.141 & $54.8 \%$ \\
\hline 54041 & WV & Lewis & rural & 370 & $28.5 \%$ & 384.90 & 0.961 & $54.5 \%$ \\
\hline 54043 & WV & Lincoln & urban & 362 & $28.4 \%$ & 437.04 & 0.828 & $56.9 \%$ \\
\hline 54045 & WV & Logan & rural & 669 & $28.3 \%$ & 453.74 & 1.475 & $65.7 \%$ \\
\hline 54049 & WV & Marion & rural & 1,295 & $28.5 \%$ & 308.74 & 4.193 & $61.6 \%$ \\
\hline 54051 & WV & Marshall & urban & 527 & $28.4 \%$ & 305.43 & 1.726 & $48.0 \%$ \\
\hline 54053 & WV & Mason & rural & 589 & $28.6 \%$ & 430.75 & 1.367 & $69.0 \%$ \\
\hline 54047 & WV & McDowell & rural & 375 & $28.2 \%$ & 533.46 & 0.703 & $64.9 \%$ \\
\hline 54055 & WV & Mercer & rural & 1,326 & $28.4 \%$ & 418.99 & 3.165 & $64.4 \%$ \\
\hline 54057 & WV & Mineral & urban & 652 & $28.4 \%$ & 327.83 & 1.987 & $75.9 \%$ \\
\hline 54059 & WV & Mingo & rural & 414 & $28.2 \%$ & 423.11 & 0.978 & $64.5 \%$ \\
\hline 54061 & WV & Monongalia & urban & 1,203 & $28.5 \%$ & 360.06 & 3.340 & $53.8 \%$ \\
\hline 54063 & WV & Monroe & rural & 441 & $28.5 \%$ & 472.75 & 0.932 & $71.1 \%$ \\
\hline 54065 & WV & Morgan & rural & 501 & $28.8 \%$ & 229.07 & 2.186 & $74.9 \%$ \\
\hline 54067 & WV & Nicholas & rural & 557 & $28.3 \%$ & 646.82 & 0.862 & $57.7 \%$ \\
\hline 54069 & WV & Ohio & urban & 933 & $28.3 \%$ & 105.82 & 8.817 & $45.1 \%$ \\
\hline 54071 & WV & Pendleton & rural & 227 & $28.6 \%$ & 696.05 & 0.327 & $67.2 \%$ \\
\hline 54073 & WV & Pleasants & rural & 152 & $28.4 \%$ & 130.10 & 1.167 & $60.6 \%$ \\
\hline 54075 & WV & Pocahontas & rural & 229 & $28.4 \%$ & 940.28 & 0.243 & $66.6 \%$ \\
\hline 54077 & WV & Preston & urban & 760 & $28.6 \%$ & 648.80 & 1.172 & $67.1 \%$ \\
\hline 54079 & WV & Putnam & urban & 905 & $28.6 \%$ & 345.67 & 2.619 & $54.3 \%$ \\
\hline 54081 & WV & Raleigh & urban & 1,825 & $28.4 \%$ & 605.35 & 3.015 & $70.0 \%$ \\
\hline 54083 & WV & Randolph & rural & 754 & $28.8 \%$ & $1,039.68$ & 0.725 & $67.4 \%$ \\
\hline 54085 & WV & Ritchie & rural & 244 & $28.6 \%$ & 451.99 & 0.540 & $64.0 \%$ \\
\hline 54087 & WV & Roane & rural & 302 & $28.2 \%$ & 483.56 & 0.624 & $61.2 \%$ \\
\hline 54089 & WV & Summers & rural & 289 & $28.3 \%$ & 360.46 & 0.801 & $68.8 \%$ \\
\hline 54091 & WV & Taylor & rural & 337 & $28.6 \%$ & 172.77 & 1.953 & $67.8 \%$ \\
\hline 54093 & WV & Tucker & rural & 179 & $28.7 \%$ & 418.92 & 0.428 & $60.4 \%$ \\
\hline 54095 & WV & Tyler & rural & 165 & $27.9 \%$ & 256.29 & 0.643 & $57.3 \%$ \\
\hline 54097 & WV & Upshur & rural & 498 & $28.5 \%$ & 354.64 & 1.403 & $55.5 \%$ \\
\hline 54099 & WV & Wayne & urban & 595 & $28.4 \%$ & 505.98 & 1.176 & $59.2 \%$ \\
\hline 54101 & WV & Webster & rural & 211 & $28.5 \%$ & 553.47 & 0.382 & $61.3 \%$ \\
\hline 54103 & WV & Wetzel & rural & 406 & $28.5 \%$ & 358.06 & 1.133 & $51.7 \%$ \\
\hline 54105 & WV & Wirt & urban & 130 & $28.5 \%$ & 232.51 & 0.558 & $62.0 \%$ \\
\hline 54107 & WV & Wood & urban & 2,107 & $28.5 \%$ & 366.26 & 5.753 & $67.7 \%$ \\
\hline 54109 & WV & Wyoming & rural & 429 & $28.2 \%$ & 499.45 & 0.858 & $65.2 \%$ \\
\hline 56001 & WY & Albany & rural & 724 & $28.5 \%$ & $4,273.84$ & 0.170 & $84.0 \%$ \\
\hline
\end{tabular}




\begin{tabular}{|c|c|c|c|c|c|c|c|c|}
\hline 56003 & WY & Big Horn & rural & 421 & $28.6 \%$ & 3137.1 & 0.134 & $91.7 \%$ \\
\hline 56005 & WY & Campbell & rural & 695 & $28.5 \%$ & $4,802.71$ & 0.145 & $86.1 \%$ \\
\hline 56007 & WY & Carbon & rural & 439 & $28.6 \%$ & $7,897.58$ & 0.056 & $88.0 \%$ \\
\hline 56009 & WY & Converse & rural & 397 & $28.7 \%$ & $4,254.88$ & 0.093 & $91.2 \%$ \\
\hline 56011 & WY & Crook & rural & 265 & $28.8 \%$ & $2,854.41$ & 0.093 & $87.6 \%$ \\
\hline 56013 & WY & Fremont & rural & 1,271 & $28.5 \%$ & $9,183.81$ & 0.138 & $84.7 \%$ \\
\hline 56015 & WY & Goshen & rural & 528 & $28.6 \%$ & $2,225.39$ & 0.237 & $92.1 \%$ \\
\hline 56017 & WY & Hot Springs & rural & 226 & $28.4 \%$ & 2004.09 & 0.113 & $94.4 \%$ \\
\hline 56019 & WY & Johnson & rural & 365 & $28.6 \%$ & $4,154.15$ & 0.088 & $90.3 \%$ \\
\hline 56021 & WY & Laramie & urban & 2,712 & $28.5 \%$ & $2,685.91$ & 1.010 & $88.6 \%$ \\
\hline 56025 & WY & Natrona & urban & 2,045 & $28.5 \%$ & $5,340.35$ & 0.383 & $87.0 \%$ \\
\hline 56027 & WY & Niobrara & rural & 104 & $28.4 \%$ & $2,626.04$ & 0.040 & $90.1 \%$ \\
\hline 56029 & WY & Park & rural & 1,279 & $28.4 \%$ & $6,942.08$ & 0.184 & $92.3 \%$ \\
\hline 56031 & WY & Platte & rural & 403 & $28.5 \%$ & $2,084.21$ & 0.193 & $91.6 \%$ \\
\hline 56033 & WY & Sheridan & rural & 1,110 & $28.7 \%$ & $2,523.99$ & 0.440 & $85.9 \%$ \\
\hline 56035 & WY & Sublette & rural & 249 & $28.4 \%$ & $4,886.54$ & 0.051 & $89.5 \%$ \\
\hline 56037 & WY & Sweetwater & rural & 868 & $28.6 \%$ & $10,426.65$ & 0.083 & $88.0 \%$ \\
\hline 56039 & WY & Teton & rural & 611 & $28.7 \%$ & $3,995.38$ & 0.153 & $87.3 \%$ \\
\hline 56041 & WY & Uinta & rural & 391 & $28.5 \%$ & $2,081.26$ & 0.188 & $78.1 \%$ \\
\hline
\end{tabular}

